\title{
Inductive game theory: A basic scenario
}

\author{
Mamoru Kaneko ${ }^{\mathrm{a}, *}$, J. Jude Kline ${ }^{\mathrm{b}}$ \\ a Institute of Policy and Planning Sciences, University of Tsukuba, Ibaraki 305-8573, Japan \\ b Department of Economics, School of Business, Bond University, Gold Coast, QLD 4229, Australia
}

\section{A R T I C L E I N F O}

\section{Article history:}

Received 1 January 2006

Received in revised form 1 July 2008

Available online 26 July 2008

\section{JEL classification:}

C70

C72

C79

\section{Keywords:}

Experience

Extensive game

Information piece

Memory function

Induction

Experiential foundation

Nash equilibrium

\begin{abstract}
A B S T R A C T
The aim of this paper is to present the new theory called "inductive game theory". A paper, published by one of the present authors with A. Matsui, discussed some part of inductive game theory in a specific game. Here, we present a more entire picture of the theory moving from the individual raw experiences, through the inductive derivation of a view, to the implications for future behavior. Our developments generate an experiential foundation for game theory and for Nash equilibrium.
\end{abstract}

(c) 2008 Elsevier B.V. All rights reserved.

\section{Introduction}

\subsection{General motivations}

In game theory and economics it is customary to assume, often implicitly and sometimes explicitly, that each player has well formed beliefs/knowledge of the game he plays. Various frameworks have been prepared for explicit analyses of this subject. However, the more basic question of where a personal understanding of the game comes from is left unexplored. In some situations such as parlour games, it might not be important to ask the source of a player's understanding. The rules of parlour games are often described clearly in a rule book. However, in social and economic situations, which are main target areas for game theory, the rules of the game are not clearly specified anywhere. In those cases, players need some other sources for their beliefs/knowledge. One ultimate source for a player's understanding is his individual experiences of playing the game. The purpose of this paper is to develop and to present a theory about the origin and emergence of individual beliefs/knowledge from the individual experiences of players with bounded cognitive abilities.

People often behave naturally and effectively without much conscious effort to understand the world in which they live. For example, we may work, socialize, exercise, eat, sleep, without consciously thinking about the structure of our social

\footnotetext{
* Corresponding author. Fax: +81 298535070.

E-mail addresses: kaneko@shako.sk.tsukuba.ac.jp (M. Kaneko), Jeffrey_Kline@bond.edu.au (J.J. Kline).
} 
situation. Nevertheless, experiences of these activities may influence our understanding and thoughts about society. We regard these experiences as important sources for the formation of individual understanding of society.

Treating particular experiences as the ultimate source of general beliefs/knowledge is an inductive process. Induction is differentiated from deduction in the way that induction is a process of deriving a general statement from a finite number of observations, while deduction is a process of deriving conclusions with the same or less logical content with well-formed inference rules from given premises. Formation of beliefs/knowledge about social games from individual experiences is typically an inductive process. Thus, we will call our theory inductive game theory, as was done in Kaneko and Matsui (1999). In fact, economic theory has had a long tradition of using arguments about learning by experiences to explain how players come to know the structure of their economy. Even in introductory microeconomics textbooks, the scientific method of analysis is discussed: collecting data, formulating hypotheses, predicting, behaving, checking, and updating. Strictly speaking, these steps are applied to economics as a science, but also sometimes, less scientifically, to ordinary peoples' activities.

Our theory formalizes some part of an inductive process of an individual decision maker. In particular, we describe how a player might use his experiences to form a hypothesis about the rules and structure of the game. In the starting point of our theory, a player has little a priori beliefs/knowledge about the structure of the particular game. Almost all beliefs/knowledge about the structure of the particular game are derived from his experiences and memories.

A player is assumed to follow some regular behavior, but he occasionally experiments by taking some trials in order to learn about the game he plays. It may be wondered how a player can act regularly or conduct experiments initially without any beliefs or knowledge. As mentioned above, many of our activities do not involve high brow analytical thoughts; we simply act. In our theory, some well defined default action is known to a player, and whenever he faces a situation he has not thought about, he chooses this action. Initially, the default action describes his regular behavior, which may interpreted as a norm in society. The experimental trials are not well developed experiments, but rather trials taken to see what happens. By taking these trials and observing resulting outcomes from them, a player will start to learn more about the other possibilities and the game overall.

The theory we propose has three main stages illustrated in Fig. 1: the (early) experimentation stage; the inductive derivation stage; and the analysis stage. This division is made for conceptual clarity and should not be confused with the rules of the dynamics. In the experimentation stage, a player accumulates experiences by choosing his regular behavior and occasionally some alternatives. This stage may take quite some time and involve many repetitions before a player moves on to the inductive stage. In the inductive derivation stage he constructs a view of the game based on the accumulated experiences. In the analysis stage, he uses his derived view to analyze and optimize his behavior. If a player successfully passes through these three stages, then he brings back his optimizing behavior to the objective situation in the form of a strategy and behaves accordingly.

In this paper, we should stop at various points to discuss some details of each of the above stages. Since, however, our intention is to give an entire scenario, we will move on to each stage sacrificing a detailed study of such a point. After passing through all three stages, the player may start to experiment again with other behaviors and the experimentation stage starts again. Experimentation is no longer early since the player now has some beliefs about the game being played. Having his beliefs, a player may now potentially learn more from his experiments. Thus, the end of our entire scenario is connected to its start.

While we will take one player through all the stages in our theory, we emphasize that other players will experiment and move through the stages also at different times or even at the same time. The precise timing of this movement is not given rigorously. In Section 7.2 we give an example of how this process of moving through these stages might occur. We emphasize

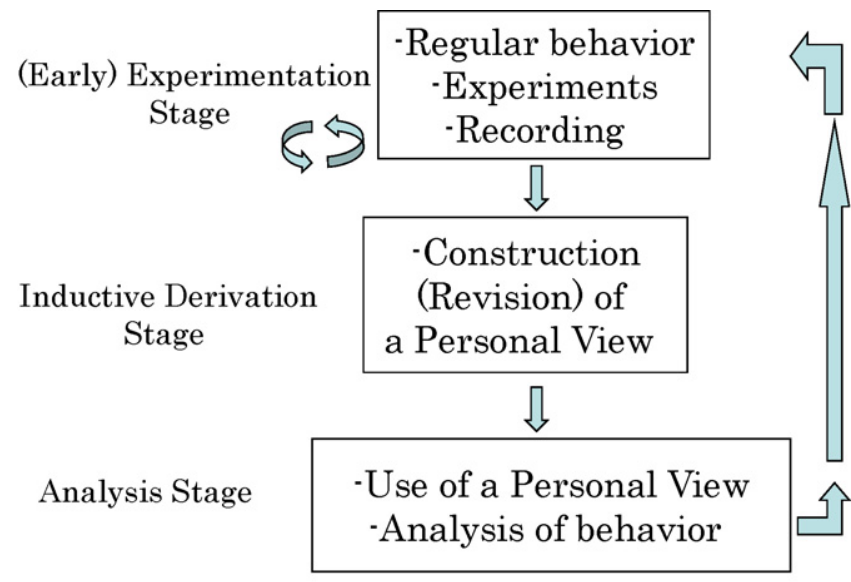

Mental-Behavioral Activities

Fig. 1. Three stages of inductive game theory. 
that experiments are still infrequent occurrences, and the regular behavior is crucial for a player to gain some information from his experiments. Indeed, if all players experiment too frequently, little would be learned.

We should distinguish our theory from some approaches in the extant game theory literature. First, we take up the typespace approach of Harsanyi (1967), which has been further developed by Mertens and Zamir (1985) and Brandenburger and Dekel (1993). In this approach, one starts with a set of parameter values describing the possible games and a description of each player's "probabilistic" beliefs about those parameters. In contrast, we do not express beliefs/knowledge either by parameters or by probabilities on them. In our approach, players' beliefs/knowledge are taken as structural expressions. Our main question is how a player derives such structural expressions from his accumulated experiences. In this sense, our approach is very different.

Our theory is also distinguished from the behavioral game theories that fall under the terms of evolution/learning/experiment (cf., Weibull, 1995; Fudenberg and Levine, 1993; Kalai and Lehrer, 1993; and more generally, Camerer, 2003) and the case-based decision theory of Gilboa and Schmeidler (1995). The behavioral game theories are typically interested in adjustment/convergence of actions to some equilibrium. They do not address questions on how a player learns the rules/structure of the game. Behavioral game theorists focus on the "rules of behavior", i.e., "strategies". Case-based decision theory looks more similar to ours. This theory focuses on how a player uses his past experiences to predict the consequences of an action in similar games. Unlike our theory, it does not discuss the emergence of beliefs/knowledge on social structures.

Rather than the above mentioned literature, our theory is reminiscent of some philosophical tradition on induction. Both Bacon (1589) and Hume (1759) regard individual experience as the ultimate source of our understanding nature, rather than society. Our theory is closer to Bacon than Hume in that the target of understanding is a structure of nature in Bacon, while Hume focussed on similarity. In this sense, the case- based decision theory of Gilboa and Schmeidler (1995) is closer to Hume. Another point relevant to the philosophy literature is that in our theory, some falsities are inevitably involved in a view constructed by a player from experiences and each of them may be difficult to be removed. Thus, our discourse does not give a simple progressive view for induction. This is close to Kuhn's (1964) discourse of scientific revolution (cf. also Harper and Schulte, 2005 for a concise survey of related works).

\subsection{Treatments of memories and inductive processes}

Here, we discuss our treatment of memory and induction in more detail. A player may, from time to time, construct a personal view to better understand the structure of some objective game. His view depends on his past interactions. The entire dynamics of a player's interactions in various objective games is conceptually illustrated in the upper figure of Fig. 2. Here, each particular game is assumed to be described by a pair $(\Gamma, \mathfrak{m})$ of an $n$-person objective extensive game $\Gamma$ and objective memory functions $\mathfrak{m}=\left(\mathfrak{m}_{1}, \ldots, \mathfrak{m}_{n}\right)$. Different superscripts here denote different objective games that a player might face, and the arrows represent the passing of time. This diagram expresses the fact that a player interacts in different games with different players and sometimes repeats the same games.

We assume that a player focuses on a particular game situation such as $\left(\Gamma^{1}, \mathfrak{m}^{1}\right)$, but he does not try to understand the entire dynamics depicted in the upper diagram of Fig. 2. The situation $\left(\Gamma^{1}, \mathfrak{m}^{1}\right)$ occurs occasionally, and we assume that the player' behavior depends only upon the situation and he notices its occurrence when it occurs. By these assumptions, the dynamics are effectively reduced into those of the lower diagram of Fig. 2. His target is the particular situation $\left(\Gamma^{1}, \mathfrak{m}^{1}\right)$. In the remainder of the paper, we denote a particular situation $\left(\Gamma^{1}, \mathfrak{m}^{1}\right)$ under our scrutiny by $\left(\Gamma^{\mathrm{o}}, \mathfrak{m}^{\mathrm{o}}\right)$, where the superscript

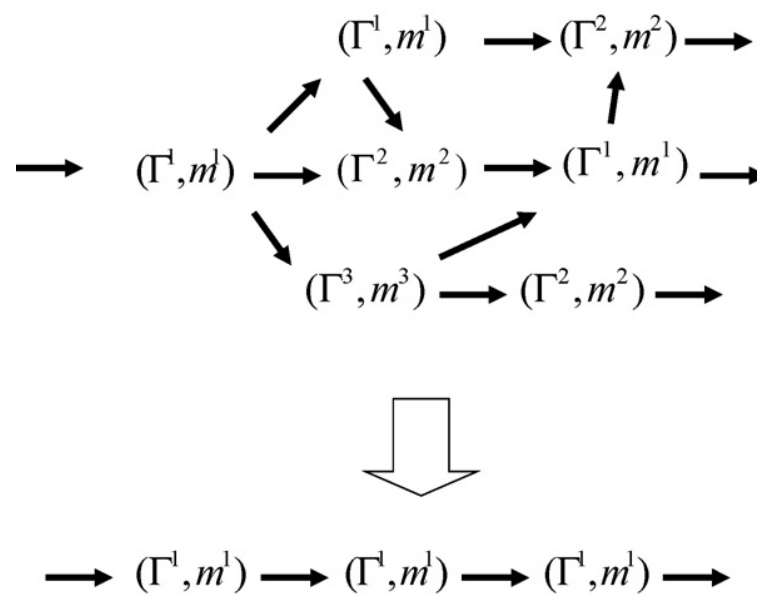

Fig. 2. Various social situations. 


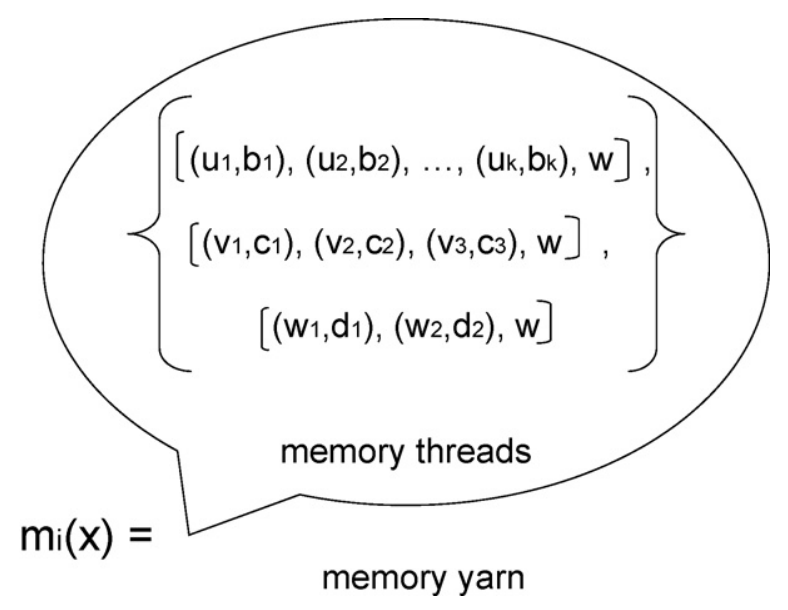

Fig. 3. Local memory-short-term memory.

"o" means "objective". We use the superscript $i$ to denote the inductively derived personal view $\left(\Gamma^{i}, \mathfrak{m}^{i}\right)$ of player $i$ about the objective situation $\left(\Gamma^{\mathrm{o}}, \mathrm{m}^{\mathrm{o}}\right)$.

The objective memory function $\mathfrak{m}_{i}^{o}$ of player $i$ describes how the raw experiences of playing $\Gamma^{\circ}$ are perceived in his mind. We refer to these memories as short-term memories and presume that they are based on his observations of information pieces and actions while he repeatedly plays $\Gamma^{\mathrm{o}}$. The "information pieces" here correspond to what in game theory are typically called "information sets", and they convey information to the player about the set of available actions at the current move and perhaps some other details about the current environment. Our use of the term "piece" rather than "set" is crucial for inductive game theory and it is elaborated on in Section 2.

An objective short-term memory $\mathfrak{m}_{i}^{\circ}(x)$ for player $i$ at his node (move) $x$ consists of sequences of pairs of information pieces and actions as depicted in Fig. 3. In this figure, a single short-term memory consists of three sequences and describes what, player $i$ thinks, might have happened prior to the node $x$ in the current play of $\Gamma^{\circ}$. In his mind, any of these sequences could have happened and the multiplicity may be due to forgetfulness. We will use the term memory thread for a single sequence, and memory yarn for the value ("set of memory threads") of the memory function at a point of time.

One role of each short-term memory value $\mathfrak{m}_{i}^{\circ}(x)$ is for player $i$ to specify an action depending upon the value while playing $\Gamma^{\mathrm{o}}$. The other role is the source for a $l$ ong-term memory, which is used by player $i$ to inductively derive a personal $\operatorname{view}\left(\Gamma^{i}, \mathfrak{m}^{i}\right)$.

The objective record of short-term memories for player $i$ in the past is a long sequence of memory yarns. A player cannot keep such an entire record; instead, he keeps short-term memories only for some length of periods. If some occur frequently enough, they change into long-term memories; otherwise, they disappear from his mind. These long-term memories remain in his mind as accumulated memories, and become the source for an inductive derivation of a view on the game. This process will be discussed in Section 3.

The induction process of player $i$ starts with a memory kit, which consists of the set of accumulated threads and the set of accumulated yarns. The accumulated threads are used to inductively derive a subjective game $\Gamma^{i}$, and the yarns may be used to construct his subjective memory function $\mathrm{m}^{i}$. This inductive process of deriving a personal view is illustrated in Fig. 4 .

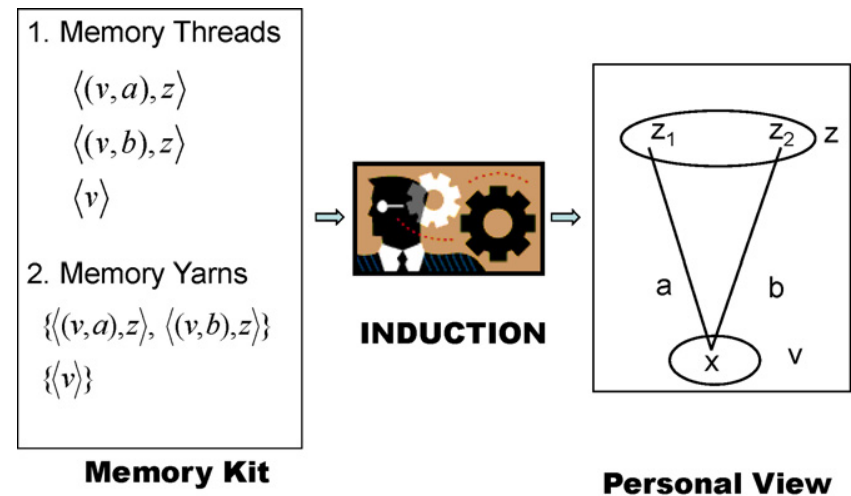

Fig. 4. Inductive derivation. 
In this paper, we consider one specific procedure for the inductive process, which we call the initial-segment procedure. This procedure will be discussed in formally in Section 4.

\subsection{The structure of the present paper}

This paper is divided into three parts:

Part I: Background, and basic concepts of inductive game theory. Sections $1-3$. Section 1 is now describing the motivation, background, and a rough sketch of our new theory. We will attempt, in this paper, to give a basic scenario of our entire theory. The mathematical structure of our theory is based on extensive games. Section 2 gives the definition of an extensive game in two senses: strong and weak. This distinction will be used to separate the objective description of a game from a player's subjective view, which is derived inductively from his experiences. Section 3 gives an informal theory of accumulating long-term memories, and a formal description of the long-term memories as a memory kit.

Part II: Inductive derivation of a personal view. Sections 4-6. In Section 4, we define an inductively derived personal view. We do not describe the induction process entirely. Rather, we give conditions that determine whether on not a personal view might be inductively derived from a memory kit. Because we have so many potential views, we define a direct view in Section 5, which turns out to be a representative of all the views a player might inductively derive (Section 6).

Part III: Decision making using an inductively derived view. Section 7-9. In this part, we consider each player's use of his derived view for his decision making. We consider a specific memory kit which allows each player to formulate his decision problem as a 1-person game. Nevertheless, this situation serves as an experiential foundation of Nash equilibrium. This Nash equilibrium result, and more general issues of decision making, are discussed in Sections 7 and 8.

Before proceeding to the formal theory in Section 2, we mention a brief history of this paper and the present state of inductive game theory. The original version was submitted to this journal in January 2006. We are writing the final version now two and a half years later in July 2008. During this period, we have made several advancements in inductive game theory, which have resulted in other papers. The results of the present paper stand alone as crucial developments in inductive game theory. Nevertheless, the connection between the newer developments and this paper need some attention. Rather than to interrupt the flow of this paper, we have chosen to give summaries and comments on the newer developments in a postscript presented as Section 9.3.

\section{Extensive games, memory, views, and behavior}

To describe a basic situation like $\left(\Gamma^{1}, \mathfrak{m}^{1}\right)$ in Fig. 2, we will use an $n$-person extensive game $\Gamma^{1}$ and memory functions $\mathfrak{m}^{1}=\left(\mathfrak{m}_{1}^{1}, \ldots, \mathfrak{m}_{n}^{1}\right)$. We follow Kuhn's (1953) formulation of an extensive game to represent $\Gamma^{1}$, except for the replacement of information sets by information pieces. ${ }^{1}$ This replacement is essential for inductive game theory. We use extensive games in the strong and weak senses to model the objective game situation and the inductively derived view of a player, which are given in Section 2.1. The memory functions $\mathfrak{m}_{1}^{1}, \ldots, \mathfrak{m}_{n}^{1}$ will be described in Section 2.2. Then, we formally define an objective description $\left(\Gamma^{1}, \mathfrak{m}^{1}\right)$ and a personal view $\left(\Gamma^{i}, \mathfrak{m}^{i}\right)$ of player $i$ in Section 2.2. In Section 2.3 we give a formal definition of a behavior pattern (strategy configuration) for the players.

\subsection{Extensive games}

Our definition of an extensive game in the strong sense differs from that of Kuhn (1953) mainly in that the information sets of Kuhn are replaced by information pieces. This difference is essential from the subjective point of view, though it is inessential from the objective point of view. An extensive game in the weak sense differs more substantially from an extensive game of Kuhn.

For notational simplicity, we sometimes make use of a function with the empty domain, which we call an empty function. When the empty domain and some (possibly nonempty) region are given, the empty function is uniquely determined.

Definition 2.1 (Extensive games). An extensive game in the strong sense $\Gamma=\left((X,<),(\lambda, W),\left\{\left(\varphi_{x}, A_{x}\right)\right\}_{x \in X},(\pi, N), h\right)$ is defined as follows:

${ }^{1}$ There are various formulations of extensive games such as in von Neumann and Morgenstern (1944), Selten (1975), Dubey and Kaneko (1984), Osborne and Rubinstein (1994) and Ritzberger (2002). Those are essentially the same formulations, while Dubey and Kaneko (1984) give a simultaneous move form. 
$\mathrm{K} 1$ (Game tree): $(X,<)$ is a finite forest (in fact, a tree by K14);

$\mathrm{K} 11: X$ is a finite non-empty set of nodes, and $<$ is a partial ordering over $X$;

K12: the set $\{x \in X: x<y\}$ is totally ordered with $<$ for any $y \in X^{2}$;

K13: $X$ is partitioned into the set $X^{D}$ of decision nodes and the set $X^{E}$ of endnodes so that every node in $X^{D}$ has at least one successor, and every node in $X^{E}$ has no successors ${ }^{3}$;

K14: $X$ has the smallest element $x_{0}$, called the root. ${ }^{4}$

K2 (Information function): $W$ is a finite set of information pieces and $\lambda: X \rightarrow W$ is a surjection with $\lambda(x) \neq \lambda(z)$ for any $x \in X^{D}$ and $z \in X^{E}$;

K3 (Available action sets): $A_{x}$ is a finite set of available actions for each $x \in X$;

$\mathrm{K} 31: A_{x}=\emptyset$ for all $x \in X^{E}$;

K32: for all $x, y \in X^{D}, \lambda(x)=\lambda(y)$ implies $A_{x}=A_{y}$;

K33: for any $x \in X, \varphi_{x}$ is a bijection from the set of immediate successors ${ }^{5}$ of $x$ to $A_{x}$;

K4 (Player assignment): $N$ is a finite set of players and $\pi: W \rightarrow 2^{N}$ is a player assignment with two conditions;

K41: $|\pi(w)|=1$ if $w \in\left\{\lambda(x): x \in X^{D}\right\}$ and $\pi(w)=N$ if $w \in\left\{\lambda(x): x \in X^{E}\right\}$;

K42: for all $j \in N, j \in \pi(w)$ for some $w \in\left\{\lambda(x): x \in X^{D}\right\}$;

K5 (Payoff functions): $h=\left\{h_{i}\right\}_{i \in N}$, where $h_{i}:\left\{\lambda(x): x \in X^{E}\right\} \rightarrow R$ is a payoff function for player $i \in N$.

Bijection $\varphi_{x}$ associates an action with an immediate successor of $x$. Game theoretically, it names each branch at each node in the tree. When $x$ is an endnode, $\varphi_{x}$ is the empty function. Since $A_{x}$ is empty, too, by K31, $\varphi_{x}$ is a bijection.

When K14 (root) is dropped, and K33 (bijection) and K5 (payoffs) are replaced by the following weaker requirements, we say that $\Gamma$ is an extensive game in the weak sense:

K33 0 : for any $x \in X, \varphi_{x}$ is a function from the set of immediate successors of $x$ to $A_{x}$.

$\mathrm{K} 5_{0}: h:\left\{\lambda(x): x \in X^{E}\right\} \rightarrow R$ is a payoff function for player $i$.

Since $X$ may not have the smallest element, $(X,<)$ is not necessarily a tree. However, $(X,<)$ is divided into several connected parts. We can prove that each maximal connected subset of $(X,<)$ is a tree. Thus, $(X,<)$ is a class of trees, i.e., a forest. For any $x \in X$, there is a unique path to $x$, i.e., each maximal set $\left\{x_{1}, \ldots, x_{m+1}\right\}$ with $x_{t}<x_{t+1}$ for $t=1, \ldots, m$ and $x_{m+1}=x$. When $x$ is an endnode, we will call the path to $x$ a play.

In an extensive game in the weak sense, an action $a$ at a node $x$ may not uniquely determine an immediate successor. See Fig. 5, which will be discussed as a derived view in Section 4.1. The converse, however, that an immediate successor determines a unique action, does hold by K330. Thus, we can define: $x{ }_{a}^{I} y$ iff $x<{ }^{I} y$ and $\varphi_{x}(y)=a$, which means that $y$ is an immediate successor of $x$ via action $a$. Then, we define $x<_{a} y$ iff there is some $y^{\prime}$ such that $x<_{a}^{I} y^{\prime}$ and $\left(y^{\prime}=y\right.$ or $\left.y^{\prime}<y\right)$.

We will use an extensive game in the strong sense as an objective description of a social situation we target, e.g., $\Gamma^{\circ}=\Gamma^{1}$ in Fig. 2. An extensive game in the weak sense will be used for a personal view inductively derived from experiences. The latter differs from the former in several respects, besides the one mentioned above. First, we take the payoffs as personal and assume that a player's personal view does not include the payoffs of other players. Hence, condition K5 is weakened to $\mathrm{K} 5{ }_{0}$ for a personal view. Dropping the root assumption and weakening K33 are more substantial changes. We will see why such changes are needed when we derive a personal view in Section 4.

For an extensive game in the weak or strong sense, condition K32 implies that the set of available actions at a node $x$ is determined by the information piece $w=\lambda(x)$. Thus, we may write $A_{w}$ or $A_{\lambda(x)}$ rather than $A_{x}$.

An extensive game in the strong sense is the same as that given in Kuhn (1953), except that we use information pieces $W$, rather than information sets. When the structure of $\Gamma$ is known, information sets are defined by information pieces, i.e., $\{x: \lambda(x)=w\}$ for $w \in W$. In this sense, our definition of an extensive game is essentially the same as Kuhn's formulation from the objective point of view. However, the replacement of information sets by information pieces is substantive from the subjective point of view for our inductive game theory.

For the purpose of comparisons, we first mention the standard interpretation of the theory of extensive games due to Kuhn (1953) (also, cf., Luce and Raiffa, 1957, Section 3.6). The interpretation is summarized as follows:

(i) (Full cognizance): each player is fully cognizant of the game structure;

(ii) (Ex ante decision): each player makes a strategy choice before the actual play of the game.

\footnotetext{
${ }^{2}$ The binary relation $<$ is called a partial ordering on $X$ iff it satisfies (i) (irreflexivity): $x \nless x$; and (ii) (transitivity): $x<y$ and $y<z$ imply $x<z$. It is a total ordering iff it is a partial ordering and satisfies (iii) (totality): $x<y, x=y$ or $y<x$ for all $x, y \in X$.

${ }^{3}$ We say that $y$ is a successor of $x$ iff $x<y$, and that $y$ is an immediate successor of $x$, denoted by $x<{ }^{I} y$, iff $x<y$ and there is no $z \in X$ such that $x<z$ and $z<y$.

${ }^{4}$ A node $x$ is called the smallest element in $X$ iff $x<y$ or $x=y$ for all $y \in X$.

${ }^{5}$ The reason for the bijection from immediate successor to actions, rather than from actions to immediate successors will be found in K33 ${ }_{0}$ below.
} 


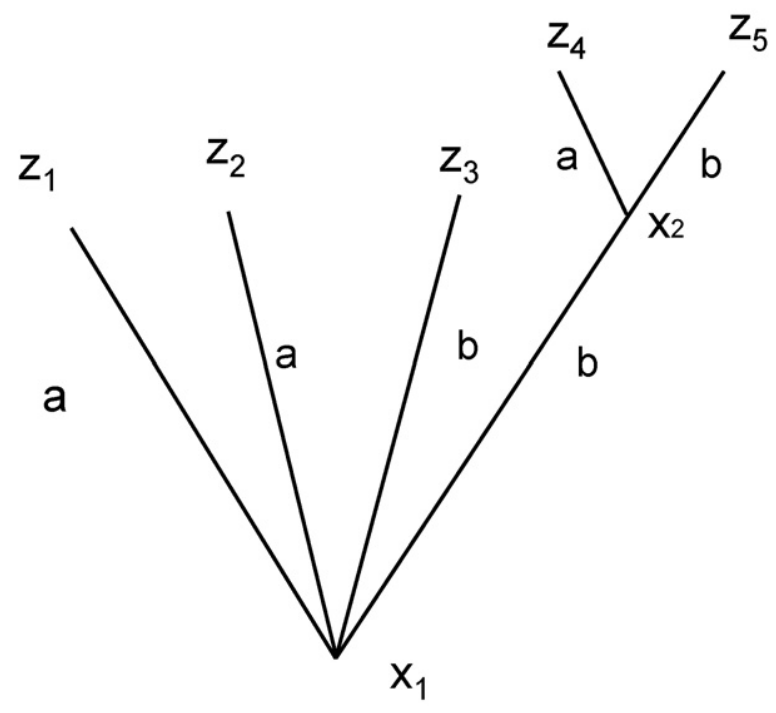

Fig. 5. Violation of condition K33.

Under (i), when a player receives an information piece $w$, he can infer the information set $\{x: \lambda(x)=w\}$ corresponding to piece $w$. Interpretation (i) is usually assumed so as to make (ii) meaningful. This will be discussed in the end of this subsection.

In the inductive context described in Section 1, the assumption (i) is dropped. Instead, players learn some part of the game structure by playing the game. Early on, a player may not infer at all the set of possible nodes having information piece $w$. To explain such differences, we use one small example of an extensive game, which we will repeatedly use to illustrate new concepts.

Example 2.1. Consider the extensive game depicted in Fig. 6. It is an example of a 2-person extensive game. Player 1 moves at the root $x_{0}$, and then at the node $x_{3}$ if it is reached. Player 2 moves at $x_{1}$ or $x_{2}$ depending on the choice of player 1 at $x_{0}$. At endnode $z_{4}$ the payoffs to players 1 and 2 are $\left(h_{1}\left(z_{4}\right), h_{2}\left(z_{4}\right)\right)=(0,1)$. The information function $\lambda$ assigns $\lambda\left(x_{0}\right)=w$, $\lambda\left(x_{1}\right)=\lambda\left(x_{2}\right)=v, \lambda\left(x_{3}\right)=u$. At the endnodes, $z_{1}, z_{2}, z_{3}, z_{4}, z_{5}$, the information function is the identity function, i.e., $\lambda\left(z_{t}\right)=z_{t}$ for $t=1, \ldots, 5$. In Kuhn's interpretation, each player has the knowledge of the game tree. In Fig. 6, for example, when player 2 receives information piece $v$, he can infer that either $x_{1}$ or $x_{2}$ is possible, which means that he knows the information set $\left\{x_{1}, x_{2}\right\}$.

Under the inductive interpretation, when player 2 receives information piece $v$, he may not come to either of the conclusions mentioned in the previous paragraph. He might not even be aware of the existence of player 1-player 1 may think that the structure could be like Fig. 5 . In such a case, piece $v$ does not imply the information set $\left\{x_{1}, x_{2}\right\}$ and the choices by player 1 either. Thus, in the inductive situation, receiving information piece $v$ may be totally different from knowing the corresponding information set.

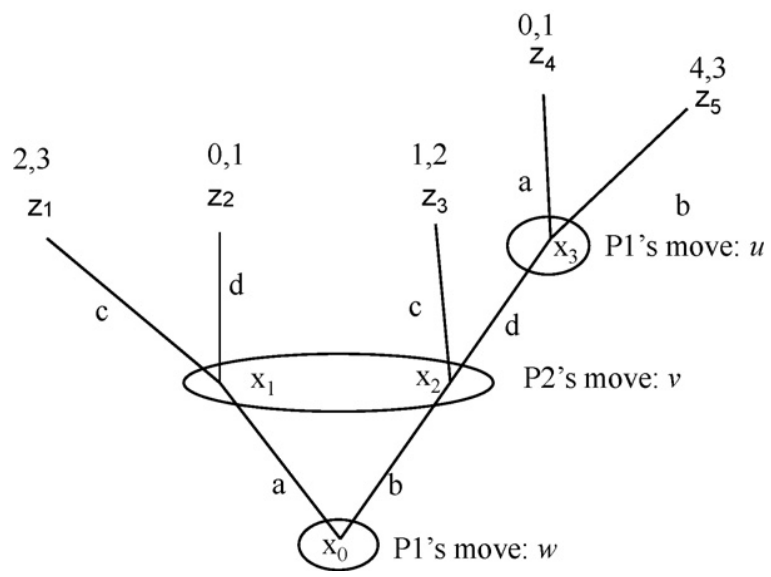

Fig. 6. 2-Person extensive game. 
The above consideration suggests that there are multiple interpretations of the knowledge a player gets from an information piece. Here, we specify the minimal content a player gets from each information piece $w$ in $\Gamma$

M1: the set $A_{w}$ of available actions;

M2: the value $\pi(w)$ of the player assignment $\pi$ if $w$ is a decision piece;

M3: his own payoff $h_{i}(w)$ (as a numerical value) if $w$ is an endpiece.

These are interpreted as being written on each piece $w$. These conditions will be discussed further when we consider some specific memory functions in Section 2.2 and the inductive derivation of a view in Section 4.

Let us return to (i) and (ii) of the standard interpretation of an extensive game given by Kuhn (1953). In our inductive game theory, since we drop the cognizance assumption (i), the ex ante decision making of (ii) does not make sense before an individual constructs a view of the game. We presume that until he constructs a view, he follows some regular behavior and makes occasional trials in an effort to learn the game he is playing. At some point of time, he will try to construct a view based on his accumulated memories of his experiences. Once a view is constructed, it may then be used by the player to construct an optimal strategy for future plays.

\subsection{Memory functions and views}

It is standard in the literature of extensive games to describe the memory ability of a player in terms of information sets (cf. Kuhn, 1953). This does not separate the role of an information piece (set) as information transmission from the role of an individual memory capability. In our inductive game theory, the treatment of various types of memories is crucial, and thus, we need an explicit formulation of individual memories in addition to an extensive game. For this reason, we introduce the concept of a memory function, which describes short-term memories of a player within a play of an extensive game.

A memory function expresses a player's short-term memory about the history of the current play of a game. Let $\Gamma=$ $\left((X,<),(\lambda, W),\left\{\left(\varphi_{x}, A_{x}\right)\right\}_{x \in X},(\pi, N), h\right)$ be an extensive game in the weak or strong sense. Recall that for each node $x \in X$, there is a unique path to $x$ which is denoted by $\left\langle x_{1}, \ldots, x_{m+1}\right\rangle$ with $x_{m+1}=x$. Also, the actions taken at $x_{1}, \ldots, x_{m}$ on the path to $x$ are uniquely determined, i.e., for each $t=1, \ldots, m$, there is a unique $a_{t} \in A_{x_{t}}$ satisfying $\varphi_{x_{t}}\left(x_{t+1}\right)=a_{t}$. We define the complete history of information pieces and actions up to $x$ by

$$
\theta(x)=\left\langle\left(\lambda\left(x_{1}\right), a_{1}\right), \ldots,\left(\lambda\left(x_{m}\right), a_{m}\right), \lambda\left(x_{m+1}\right)\right\rangle .
$$

The history $\theta(x)$ consists of observable elements for players, while the path $\left\langle x_{1}, \ldots, x_{m+1}\right\rangle$ to $x$ consists of unobservables for players. Memories will be defined in terms of these observable elements.

A short-term memory consists of memory threads, which look somewhat like the historical sequence $\theta(x)$. However, we allow a player to be forgetful, which is expressed by incomplete threads or multiple threads. Formally, a memory thread is a finite sequence

$$
\mu=\left\langle\left(v_{1}, a_{1}\right), \ldots,\left(v_{m}, a_{m}\right), v_{m+1}\right\rangle,
$$

where

$$
v_{t} \in W, a_{t} \in A_{v_{t}} \quad \text { for all } t=1, \ldots, m \text { and } v_{m+1} \in W .
$$

Each component $\left(v_{t}, a_{t}\right)(t=1, \ldots, m)$ or $v_{m+1}$ in $\mu$ is called a memory knot. A finite nonempty set of memory threads is called a memory yarn. See Fig. 3 for an illustration of these concepts. Now, we have the definition of a memory function.

Definition 2.2 (Memory functions). We say that a function $\mathfrak{m}_{i}$ is a memory function of player $i$ iff for each node $x \in X_{i}=\{x \in X$ : $i \in \pi \cdot \lambda(x)\}, \mathfrak{m}_{i}(x)$ is a memory yarn satisfying:

$$
w=\lambda(x) \text { for all }\langle\xi, w\rangle \in \mathfrak{m}_{i}(x) .
$$

The memory function $\mathfrak{m}_{i}$ gives a memory yarn consisting of a finite number of memory threads at each node for player $i$. The multiplicity of threads in a yarn describes uncertainty at a point in time about the past.

In Fig. 3, the memory yarn $\mathfrak{m}_{i}(x)$ consists of three memory threads. The first one is a long one, the second and third are memory threads of short lengths. Condition (2.4) states that the tails of any memory threads at a node $x$ are identical to the correct piece $w=\lambda(x)$. This is interpreted as meaning that the player correctly perceives the current information piece.

Here, we mention four classes of memory functions and one specific one. In the first memory function, which is the Self-scope Perfect Recall memory function, player $i$ recalls what information he received during the current game and what actions he took, but nothing about the other players. For this example, we define player $i$ 's own history: for a node $x \in X_{i}$, let $\theta(x)=\left\langle\left(\lambda\left(x_{1}\right), a_{1}\right), \ldots,\left(\lambda\left(x_{m}\right), a_{m}\right), \lambda\left(x_{m+1}\right)\right\rangle$, and let $\left\langle x_{k_{1}}, \ldots, x_{k_{l}}, x_{k_{l+1}}\right\rangle$ be the $i$-part of $\left\langle x_{1}, \ldots, x_{m}, x_{m+1}\right\rangle$, i.e., the maximal subsequence of nodes in the path $\left\langle x_{1}, \ldots, x_{m}, x_{m+1}\right\rangle$ to $x$ satisfying $i \in \pi \cdot \lambda\left(x_{k_{t}}\right)$ for $t=1, \ldots, l+1$. Then we define player $i$ 's (objective) history of information pieces and actions up to $x$ by

$$
\theta(x)_{i}=\left\langle\left(\lambda\left(x_{k_{1}}\right), a_{k_{1}}\right), \ldots,\left(\lambda\left(x_{k_{l}}\right), a_{k_{l}}\right), \lambda\left(x_{k_{l+1}}\right)\right\rangle .
$$


(1) Self-scope ${ }^{6}$ perfect-recall memory function: It is formulated as follows:

$$
\mathfrak{m}_{i}^{\mathrm{spr}}(x)=\left\{\theta(x)_{i}\right\} \quad \text { for each } x \in X_{i} .
$$

With the memory function $\mathrm{m}_{i}^{\mathrm{spr}}$, player $i$ recalls his own information pieces and actions taken in the current play of the game. This memory function will have a special status in the discourse of this paper.

In Fig. 6, the memory function $\mathrm{m}_{1}^{\mathrm{spr}}$ for player 1 is given as:

$$
\begin{aligned}
& \mathfrak{m}_{1}^{\mathrm{spr}}\left(x_{0}\right)=\{\langle w\rangle\}, \text { and } \mathfrak{m}_{1}^{\mathrm{spr}}\left(x_{3}\right)=\{\langle(w, b), u\rangle\} \\
& \mathfrak{m}_{1}^{\mathrm{spr}}\left(z_{t}\right)=\left\{\left\langle(w, a), z_{t}\right\rangle\right\} \text { for } t=1,2, \text { and } \mathfrak{m}_{1}^{\mathrm{spr}}\left(z_{3}\right)=\left\{\left\langle(w, b), z_{3}\right\rangle\right\} ; \\
& \mathfrak{m}_{1}^{\mathrm{spr}}\left(z_{4}\right)=\left\{\left\langle(w, b),(u, a), z_{4}\right\rangle\right\} \text { and } \mathfrak{m}_{1}^{\mathrm{spr}}\left(z_{5}\right)=\left\{\left\langle(w, b),(u, b), z_{5}\right\rangle\right\} .
\end{aligned}
$$

At node $x_{3}$, player 1 receives piece $u$ and recalls his choice $b$ at $w$. By the minimal requirement M1, he knows the available actions $A_{w}=\{a, b\}$ and $A_{u}=\{a, b\}$. Without adding any other source than $\mathrm{m}_{1}^{\text {spr }}$, player 2 does not appear in the scope of player 1. It will be discussed that Fig. 5. is an inductively derived view in this example.

The next example is the Markov memory function. As its name suggests, a player forgets the entire history after he makes a decision.

(2) Markov memory function: It is formulated as

$$
\mathfrak{m}_{i}^{\mathrm{M}}(x)=\{\lambda(x)\} \quad \text { for each } x \in X_{i} .
$$

It gives only the present information piece. Nonetheless, by the minimal requirement M1, the player can extract his available action set $A_{\lambda(x)}$ whenever he receives an information piece $\lambda(x)$.

For both $\mathfrak{m}_{i}^{\mathrm{spr}}$ and $\mathfrak{m}_{i}^{\mathrm{M}}$, we would have no difficulty in presuming that each player only receives his own information pieces and gets the minimal information described by M1, M2 and M3. As we will see now, some other memory functions provide a player with information about some other players' information pieces and actions. The first such memory function is the perfect information memory function.

(3) Perfect-information memory function: This is formulated as

$$
\mathfrak{m}_{i}^{\mathrm{PI}}(x)=\{\theta(x)\} \quad \text { for each } x \in X_{i} .
$$

Recall that $\theta(x)$ is given by (2.1). Thus, if player $i$ has this memory function, he recalls the perfect history even including the other players' pieces. By M1 and M2, he also knows the available actions and the player who moves at each decision piece.

There are at least two possible interpretations of how he comes to know the perfect history. One interpretation is that player $i$ observes other players' moves as the game is played. Another interpretation is that player $i$ 's information pieces contain the complete history, i.e., $\theta(x)$ is written on piece $\lambda(x)$. Under either interpretation, a player gets more than the minimal amount of information described in M1-M3.

The next memory function typically gives a player less information than the perfect information memory function.

(4) Classical memory function: This memory function is formulated as:

$$
\mathfrak{m}_{i}^{\mathrm{C}}(x)=\left\{\theta(y): y \in X_{i} \text { for each } \lambda(y)=\lambda(x)\right\} \text { for each } x \in X_{i} .
$$

Observe that this function gives player $i$ the set of complete histories up to nodes with his current information piece. The multiplicity of memory threads can be interpreted as some ambiguity about the past. This memory function can also be interpreted in the ways suggested for $\mathfrak{m}_{i}^{\mathrm{PI}}$. We should mention yet another interpretation which is the motivation for the name "classical" memory function. In this interpretation, player $i$ knows the structure of the extensive game. Consequently, he can infer the set of possible complete histories compatible with the present information piece. The classical memory function together with this interpretation is less compatible with our inductive game theory than the other memory functions. Since it is still mathematically allowed and is closer to the classical game theory, we consider it.

The general definition of a memory function allows it to even involve false components. We give one example of false memories using the following simple extensive game. Consider the 1 -person extensive game $\left(\Gamma, \mathfrak{m}_{1}\right)$ depicted as Fig. 7 with the identity information function.

\footnotetext{
${ }^{6}$ We have chosen the name "self-scope" to mean that he has only himself in his scope. Of course we allow for perfect recall memory functions where the player has other player's in his scope.
} 
$z_{1}$

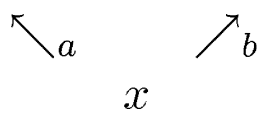

Fig. 7. False memory.

A false memory function $\mathfrak{m}_{1}$ is given as:

$$
\mathfrak{m}_{1}(x)=\{\langle x\rangle\}, \quad \mathfrak{m}_{1}\left(z_{1}\right)=\left\{\left\langle(x, a), z_{1}\right\rangle\right\} \quad \text { and } \quad \mathfrak{m}_{1}\left(z_{2}\right)=\left\{\left\langle(x, a), z_{2}\right\rangle\right\} .
$$

This $\mathfrak{m}_{1}$ takes a false value at $z_{2}$, at which player 1 incorrectly recalls having chosen $a$ at $x$ though he actually chose $b$ at $x$.

Having described an extensive game and memory functions, we now have the basic ingredients for objective descriptions and subjective personal views.

(Objective description): A pair $\left(\Gamma^{\circ}, \mathfrak{m}^{0}\right)$ is called an objective description iff $\Gamma^{\circ}$ is an extensive game in the strong sense and $\mathfrak{m}^{\mathrm{o}}=\left(\mathfrak{m}_{1}^{\mathrm{o}}, \ldots, \mathfrak{m}_{n}^{\mathrm{o}}\right)$ is an $n$-tuple of memory functions in $\Gamma^{\mathrm{o}}$.

The objective description is not available to a player. We use the superscript $o$ to denote the objective description. We will put a superscript $i$ to denote a personal view of player $i$.

(Personal view): A pair $\left(\Gamma^{i}, \mathfrak{m}^{i}\right)$ is a personal view for player $i$ iff $\Gamma^{i}$ is an extensive game in the weak sense specifying only the payoff function of player $i$, and $\mathfrak{m}^{i}$ is a memory function for player $i$ in $\Gamma^{i}$.

A personal view $\left(\Gamma^{i}, \mathfrak{m}^{i}\right)$ of player $i$ describes the game player $i$ believes he is playing. Since his belief is based on his experiences, we do not include the memory functions or payoffs of other players. We regard payoff values and memory values as personal. ${ }^{7}$

\subsection{Behavior patterns}

Let $\Gamma=\left((X,<),(\lambda, W),\left\{\left(\varphi_{X}, A_{X}\right)\right\}_{x \in X},(\pi, N), h\right)$ be an extensive game in the weak or strong sense and let $\mathfrak{m}_{i}$ be a memory function for player $i \in N$. The extensive game and memory function may be either the objective description or a personal view. We give a definition of a behavior pattern to be applied to both cases.

We say that a function $\sigma_{i}$ on the set of nodes $X_{i}^{D}:=\left\{x \in X^{D}: i \in \pi \cdot \lambda(x)\right\}$ is a $b$ ehavior pattern (strategy) of player $i$ iff it satisfies conditions (2.12) and (2.13):

$$
\begin{aligned}
& \text { for all } x \in X_{i}^{D}, \sigma_{i}(x) \in\left\{a \in A_{x}: \varphi_{x}(y)=a \text { for some } y \in X\right\} ; \\
& \text { for all } x, y \in X_{i}^{D}, \mathfrak{m}_{i}(x)=\mathfrak{m}_{i}(y) \text { implies } \sigma_{i}(x)=\sigma_{i}(y) .
\end{aligned}
$$

Condition (2.12) means that a behavior pattern $\sigma_{i}$ prescribes an action leading to some decision node. This slightly complicated statement is required since $\Gamma$ may be of the weak sense. ${ }^{8}$ Condition (2.13) means that a strategy depends upon local memories. These are standard conditions for the definition of a strategy. We denote, by $\Sigma_{i}$, the set of all behavior patterns for player $i$ in $\Gamma$. We say that an $n$-tuple of strategies $\sigma=\left(\sigma_{1}, \ldots, \sigma_{n}\right)$ is a profile of behavior patterns.

We use the term behavior pattern (strategy) to acknowledge that the behavior of a player may initially represent some default behavior with no strategic considerations. Once, a player has gathered enough information about the game, his behavior may become strategic. This will be discussed in a remark in the end of Section 3.2.

In order to evaluate a behavior pattern, we introduce the concepts of compatible endnodes and compatible endpieces. All evaluations of strategies in this paper will be done in terms of compatible endpieces. Each behavior profile $\sigma=\left(\sigma_{1}, \ldots, \sigma_{n}\right)$ determines the set of compatible endnodes:

$$
z(\sigma)=\left\{z \in X^{E}: \theta(z)=\left\langle\left(\lambda\left(x_{1}\right), \sigma\left(x_{1}\right)\right), \ldots,\left(\lambda\left(x_{k}\right), \sigma\left(x_{k}\right)\right), \lambda\left(x_{k+1}\right)\right\rangle \text { for the path }\left\langle x_{1}, \ldots, x_{k}, x_{k+1}\right\rangle \text { to } z\right\} .
$$

Thus, the actions in the history $\theta(z)$ were prescribed by the behavior profile $\sigma=\left(\sigma_{1}, \ldots, \sigma_{n}\right)$. Each behavior profile $\sigma$ also determines the set of compatible endpieces:

$$
\lambda(\sigma)=\{w: \lambda(x)=w \text { for somex } \in z(\sigma)\} .
$$

\footnotetext{
${ }^{7}$ As stated several times, we regard this as an alternative assumption adopted in the present discourse. This can be extended to include other players.

${ }^{8}$ If $\varphi_{x}$ is a surjection then $\left\{a \in A_{x}: \varphi_{x}(y)=y\right.$ for some $\left.y \in X\right\}=A_{x}$. However, since a personal view may satisfy only K $33_{0}$ we require this condition.
} 
When $\Gamma$ is an extensive game in the strong sense, $z(\sigma)$ and $\lambda(\sigma)$ are singleton sets. However, for extensive games in the weak sense, these sets may have multiple elements.

\section{Bounded memory abilities and accumulation of short-term memories}

In this section, we first define a domain of accumulation of short-term memories. This definition is based on the presumption that a player has a quite restricted memory capability. Theoretically, however, there are still many other possibilities. In Section 3.2, we will give one informal theory about the accumulation of short-term memories as long-term ones. This informal theory suggests a particular domain which we call the active domain, which turns out to be linked to Nash equilibrium behavior, as will be shown in Section 7.2. Informal and premathematical discussions of this type are intended to provoke further discussions and debates over the appropriate domain(s) for consideration.

\subsection{The objective recurrent situation and domains of accumulation of local memories}

Let an extensive game $\Gamma^{0}=\left(\left(X^{0},<^{0}\right),\left(\lambda^{0}, W^{0}\right),\left\{\left(\varphi_{x}^{0}, A_{x}^{0}\right)\right\}_{X \in X},\left(\pi^{0}, N^{0}\right), h^{0}\right)$ in the strong sense and a profile $\mathfrak{m}^{0}=$ $\left(\mathfrak{m}_{1}^{o}, \ldots, \mathfrak{m}_{n}^{0}\right)$ of memory functions be the description of the objective situation. The present purpose is to consider the accumulation of memories from playing in $\left(\Gamma^{\mathrm{o}}, \mathfrak{m}^{\mathrm{o}}\right)$ repeatedly.

From the objective point of view, an individual player, $i$, has been experiencing short-term memories:

$$
\begin{array}{r}
\cdots\left|\mathfrak{m}_{i}\left(x_{1}^{t}\right), \ldots, \mathfrak{m}_{i}\left(x_{\ell_{t}}^{t}\right)\right| \mathfrak{m}_{i}\left(x_{1}^{t+1}\right), \ldots, \mathfrak{m}_{i}\left(x_{\ell_{t+1}}^{t+1}\right) \mid \cdots \\
\left(\Gamma^{\mathrm{o}}, \mathfrak{m}^{\mathrm{o}}\right) \text { at } t \quad\left(\Gamma^{\mathrm{o}}, \mathfrak{m}^{\mathrm{o}}\right) \text { at } t+1
\end{array}
$$

where $\left\langle x_{1}^{t}, \ldots, x_{\ell_{t}}^{t}\right\rangle$ is the realized sequence of player $i$ 's nodes in the occurrence of $\left(\Gamma^{o}, \mathfrak{m}^{0}\right)$ at time $t$. Due to bounded memory, player $i$ will only accumulate some part of these as long-term memories.

In the extensive game $\left(\Gamma^{\mathrm{o}}, \mathrm{m}^{\mathrm{o}}\right)$, the domain of accumulation for player $i$ is a non-empty subset $D_{i}$ of the set $X_{i}^{\mathrm{o}}=\left\{x \in X^{\mathrm{o}}\right.$ : $\pi^{\circ}(x) \ni i$ of nodes for player $i$. Player $i$ is relevant in his own domain $D_{i}$ iff $D_{i}$ contains at least one decision node for player $i$. This definition will be important later in this paper.

A memory kit $\left(T_{D_{i}}, \mathcal{Y}_{D_{i}}\right)$ for domain $D_{i}$ is given by

$$
T_{D_{i}}=\bigcup_{x \in D_{i}} \mathfrak{m}_{i}^{\mathrm{o}}(x) ; \quad \text { and } \quad \mathcal{Y}_{D_{i}}=\left\{\mathfrak{m}_{i}^{\mathrm{o}}(x): x \in D_{i}\right\}
$$

A memory kit is determined by both the domain of accumulation $D_{i}$ and the objective memory function $\mathrm{m}_{i}^{\circ}$ of player $i$. It will be the source for an inductive construction of a personal view. The set $T_{D_{i}}$ of memory threads is used to construct a skeleton of the tree for a personal view. The set $\mathcal{Y}_{D_{i}}$ of yarns is used to construct a perceived memory function. Mathematically speaking, the latter set gives the former, but we keep those two sets to emphasize that they have different usages.

For a memory kit, we assume that player $i$ has accumulated some incidences of short-term memories as both threads and yarns. However, a kit includes neither a full record of short-term memories nor frequencies. In Section 3.2, we will discuss one rationale for this treatment.

Here, we give three domains of accumulation. The first two are trivial ones, and the third example is the one we are going to explore in this paper.

(1) Full domain: This is simply given as the entire set $D_{i}^{\mathrm{F}}=X_{i}^{\mathrm{o}}$ of player $i$ 's nodes. When the game is small, is repeated often enough and also when the accumulation ability of player $i$ is strong enough, this domain may be appropriate.

(2) Cane domain: A cane domain is a complete set of nodes for player $i$ on one play. Formally, let $\left\langle x_{0}, \ldots, x_{m}\right\rangle$ be the path to an endnode $x_{m}$. Then the cane domain of player $i$ to $x_{m}$ is given as $\left\{x_{0}, \ldots, x_{m}\right\} \cap X_{i}^{\mathrm{o}}$. A cane domain may arise if every player behaves always following some regular behavior pattern with no deviations.

Now, let $\sigma^{\mathrm{o}}=\left(\sigma_{1}^{\mathrm{o}}, \ldots, \sigma_{n}^{0}\right)$ be a profile of behavior patterns in the extensive game $\left(\Gamma^{\mathrm{o}}, \mathrm{m}^{\mathrm{o}}\right)$. Then, this $\sigma^{\mathrm{o}}$ determines a unique path to an endnode. Hence, the cane domain for player $i$ is uniquely determined, which is denoted by $D_{i}^{\mathrm{c}}\left(\sigma^{\mathrm{o}}\right)$. Using this concept, we can define the active domain relative to a profile of behavior patterns.

(3) Active domain: The active domain relative to a profile $\sigma^{\circ}=\left(\sigma_{1}^{o}, \ldots, \sigma_{n}^{o}\right)$ of behavior patterns for player $i$ is given as

$$
D_{i}^{\mathrm{A}}\left(\sigma^{\mathrm{o}}\right)=\bigcup_{\sigma_{i} \in \Sigma_{i}^{\mathrm{o}}} D_{i}^{\mathrm{c}}\left(\sigma_{i}, \sigma_{-i}^{\mathrm{o}}\right) .
$$

Here, $\Sigma_{i}^{\mathrm{o}}$ is the set of all behavior patterns for player $i$ in $\left(\Gamma^{\mathrm{o}}, \mathrm{m}^{\mathrm{o}}\right)$ and $\left(\sigma_{i}, \sigma_{-i}^{\mathrm{o}}\right)$ is the profile obtained from $\sigma^{\mathrm{o}}$ by substituting $\sigma_{i}$ for $\sigma_{i}^{\mathrm{o}}$ in $\sigma^{\mathrm{o}}$. That is, the active domain $D_{i}^{\mathrm{A}}\left(\sigma^{\mathrm{O}}\right)$ is the set of nodes for player $i$ that are reached by unilateral deviations of player $i$. 
For a unified treatment of the above domains, we introduce one definition. We say that a domain $D_{i}$ for player $i$ is $c$ losed iff $D_{i}$ is expressed as some union of cane domains of player $i$. The above three examples of domains are closed. A domain which is not closed is the set $X^{O E}$ of endnodes.

Example 3.1. Let us continue with the example of Fig. 6. Let the regular behavior be given by $\sigma_{1}^{o}\left(x_{0}\right)=\sigma_{1}^{o}\left(x_{3}\right)=a$ and $\sigma_{2}^{o}\left(x_{1}\right)=\sigma_{2}^{o}\left(x_{2}\right)=c$. The cane domain and active domain of player 1 determined by $\sigma^{0}$ are given as

$$
D_{1}^{\mathrm{c}}\left(\sigma^{\mathrm{o}}\right)=\left\{x_{0}, z_{1}\right\} \text { and } D_{1}^{\mathrm{A}}\left(\sigma^{\mathrm{o}}\right)=\left\{x_{0}, z_{1}, z_{3}\right\} \text {. }
$$

The full domain is simply given as $D_{1}^{\mathrm{F}}=X_{1}^{\mathrm{o}}=\left\{x_{0}, x_{3}, z_{1}, z_{2}, z_{3}, z_{4}, z_{5}\right\}$.

The memory kit of player 1 depends also on his objective memory function $\mathfrak{m}_{1}^{0}$. For the three domains mentioned above, the Markov and SPR memory functions, we have a total of six memory kits. We mention two and leave the reader to consider the other four.

For the SPR memory function $\mathfrak{m}_{1}^{o}=\mathfrak{m}_{1}^{\mathrm{spr}}$ and the cane domain, we have $T_{D_{1}^{c}\left(\sigma^{0}\right)}=\left\{\langle w\rangle,\left\langle(w, a), z_{1}\right\rangle\right\}$, and $\mathcal{Y}_{D_{1}^{c}\left(\sigma^{0}\right)}=$ $\left\{\{\langle w\rangle\},\left\{\left\langle(w, a), z_{1}\right\rangle\right\}\right\}$.

For the Markov memory function $\mathfrak{m}_{1}^{o}=\mathfrak{m}_{1}^{\mathrm{M}}$ and the active domain, we have $T_{D_{1}^{\mathrm{A}}\left(\sigma^{\circ}\right)}=\left\{\langle w\rangle,\left\langle z_{1}\right\rangle,\left\langle z_{3}\right\rangle\right\}$ and $\mathcal{Y}_{D_{1}^{\mathrm{A}}\left(\sigma^{0}\right)}=$ $\left\{\{\langle w\rangle\},\left\{\left\langle z_{1}\right\rangle\right\},\left\{\left\langle z_{3}\right\rangle\right\}\right\}$.

\subsection{An informal theory of behavior and accumulation of memories}

Our mathematical theory starts with a memory kit. Behind a memory kit, there is some underlying process of behavior and accumulation of short-term memories. We now describe one such underlying process informally, which justifies the active domain of accumulation. This description is given in terms of some informal postulates.

(1) Postulates for behavior and trials: The first postulate is the rule-governed behavior of each player in the recurrent situation $\ldots,\left(\Gamma^{\mathrm{o}}, \mathfrak{m}^{\mathrm{o}}\right), \ldots,\left(\Gamma^{\mathrm{o}}, \mathfrak{m}^{\mathrm{o}}\right), \ldots$

Postulate BH1 (Regular behavior): Each player typically behaves regularly following his behavior pattern $\sigma_{i}^{\circ}$.

Player $i$ may have adopted his regular behavior for some time without thinking, perhaps since he found it worked well in the past or he was taught to follow it. Without assuming regular behavior and/or patterns, a player may not be able to extract any causal pattern from his experiences. In essence, learning requires some regularity.

To learn some other part than that regularity experienced in the extensive game, the players need to make some trial deviations. We postulate that such deviations take place in the following manner.

Postulate BH2 (Occasional deviations): Once in a while (infrequently), each player unilaterally and independently makes a trial deviation $\sigma_{i} \in \Sigma_{i}^{o}$ from his regular behavior $\sigma_{i}^{o}$ and then returns to his regular behavior.

Early on, such deviations may be unconscious or not well thought out. Nevertheless, a player might find that a deviation leads to a better outcome, and he may start making deviations consciously in the future. Once he has become conscious of his behavior-deviation, he might make more and/or different trials.

The set of trial deviations for a player is not yet well specified. In the remainder of this paper, we explore one extreme case where he tries every possible behavior. The following postulate is made for simplicity in our discourse and since it connects our theory to standard game theory.

Postulate BH3 (All possible trials): Each player experiments over all his possible behaviors.

Postulate BH3 is an extreme case that each player tries all his alternative behaviors. We do not take this as basic. The choice of a smaller set of trial deviations is very relevant, since a player might not have prior knowledge of his available behaviors.

(2) Epistemic postulate: Each player may learn something through his regular behavior and deviations. What he learns in an instant is described by his short-term memory. For the transition from short-term memories to long-term memories, there are various possibilities. Here we list some postulates based on bounded memory abilities that suggest only the active domain of accumulation.

The first postulate states that if a short-term memory does not occur frequently enough, it will disappear from the mind of a player. We give this as a postulate for a cognitive bound on a player.

Postulate EP1 (Forgetfulness): If experiences are not frequent enough, then they would disappear from a player's mind. 
This is a rationale for not assuming that a player has a full record of short-term memories, as well as for the term "shortterm memory". This explains also the assumption that he cannot keep the relative frequency of a short-term memory: it may remain for some short periods, but if it is not reinforced by other occurrences or the player is very conscious, they may disappear from his mind, i.e., many disappear. This means that a memory remaining after some time loses relative positions with other memories and is isolated. Hence, it is difficult to calculate its frequency relative to others.

In the face of the cognitive bound, only some memories become lasting. The first type of memories that become lasting are the regular ones since they occur quite frequently. The process of making a memory last by repetition is known as habituation.

Postulate EP2 (Habituation): A short-term (local) memory becomes lasting as a long-term memory in the mind of a player by habituation, i.e., if he experiences something frequently enough, it remains in his memory as a long-term memory even without conscious effort.

By EP2, when all players follow their regular behavior patterns, the short-term memories given by them will become long-term memories by habituation.

The remaining possibilities for long-term memories are the memories of trials made by some players. We postulate that a player may consciously spend some effort to memorize the outcomes of his own trials.

Postulate EP3 (Conscious memorization effort): A player makes a conscious effort to memorize the result of his own trials. These efforts are successful if they occur frequently enough relative to his trials.

Postulate EP3 means that when a player makes a trial deviation, he also makes a conscious effort to record his experience in his long-term memory. These memories are more likely to be successful if they are repeated frequently enough relative to his trials. Since the players are presumed to behave independently, the trial deviations involving multiple players will occur infrequently, even relative to one player's trials. Thus, the memories associated with multiple players' trials do not remain as long-term memories. This has the implication that our experiential foundation is typically incompatible with the subgame perfect concept of Selten (1975), which will be discussed again in Section 9.

In sum, postulates EP1 to EP3 and BH1 to BH3 suggest that we can concentrate on the active domain of a player.

Some other domains such as a cane domain and the full domain might emerge as candidates in slightly different situations. For example, if no trials are made, then EP2 (Habituation) gives the cane domain corresponding to $\sigma^{\circ}$. Alternatively, if the game is small enough and if it is repeated enough, then each player has experienced every outcome. And if he has an ability to recall all the incidences, then we would get the full domain. The additional assumption of full recall seems plausible for small games.

Remark (Default decision and all the possible behaviors): One may criticize our treatments in that:

(1) $\sigma_{i}^{o}$ has the total domain $X_{i}^{o}$ and

(2) $\sigma_{i}$ varies over the entire $\Sigma_{i}^{\mathrm{o}}$ of (3.3),

since these might conflict with the assumption of no a priori knowledge of the structure of the game for player $i$.

We can answer (1) by interpreting one action at every decision node as a default action. When a player receives an unknown (unfamiliar) information piece, he just takes the default action. This assumption avoids a player's need to plan for his behavior over the entire domain.

We take (2) as a legitimate criticism, particularly, when the game is large. We have chosen (3.3) as a working assumption in this paper.

\section{Inductively derived views}

In this section, we give a definition of an inductively derived (personal) view, which we abbreviate as an i.d.view. Here, player $i$ uses only his memory kit $\left(T_{D_{i}}, \mathcal{Y}_{D_{i}}\right)$ as a summary of his experiences to construct an i.d.view. Before the definition, we talk about our basic principles to be adopted in this paper. After the definition, we will consider various examples to see the details of the definition.

\subsection{Observables, observed, and additional components}

The central notion in inductive game theory is the process of inductive inferences. An inductive inference is distinguished from a deductive inference in that the former allows some generalization of observations by adding some hypothetical components, while the latter changes expressions following well-formed inference rules and keeps the same or less contents. A player, $i$, having a memory kit $\left(T_{D_{i}}, \mathcal{Y}_{D_{i}}\right)$ may add some hypothetical components to the kit in his inductive process to develop a personal view. 
The need for this addition of hypothetical components may be found in the assumption that a player can only observe some elements of the objective extensive game $\Gamma^{\circ}$. As remarked in Section 2.2, only information pieces and actions are observable for each player, while nodes are hypothetical and unobservables. In addition, many or some pieces and actions do not end up in the memory kit. Pieces and actions only along some of the paths in a game tree are more likely observed for players. Moreover, the bounds on their memory capabilities will allow them to accumulate memories of only some of what they have observed. The memory kit $\left(T_{D_{i}}, \mathcal{Y}_{D_{i}}\right)$ for player $i$ is the collection of observed parts effectively remaining in the mind of player $i$.

Since player $i$ describes his view $\left(\Gamma^{i}, \mathfrak{m}^{i}\right)$ as an extensive game in the weak sense with a memory function, he needs to invent a tree structure by adding hypothetical nodes. In this sense he already goes beyond deductive inferences. To construct a coherent view, a player may add other components, e.g., more information pieces, actions, and possible histories to his memories. In this paper, however, we adhere to the basic principle that only elements in the memory kit $\left(T_{D_{i}}, \mathcal{Y}_{D_{i}}\right)$ can be used as the observables in $\left(\Gamma^{i}, \mathrm{~m}^{i}\right)$. In Section 4.2, we will adopt a specific inductive process called the initial-segment procedure and use this procedure to define an i.d.view. With this procedure, a player forms the underlying skeletal structure of his view by adding hypothetical nodes.

\subsection{Definition and examples}

Now, consider the recurrent situation of $\left(\Gamma^{\mathrm{o}}, \mathrm{m}^{\mathrm{o}}\right)$ illustrated in Fig. $2 . \quad$ Here, $\Gamma^{\mathrm{o}}=$ $\left(\left(X^{\mathrm{o}},<^{\mathrm{o}}\right),\left(\lambda^{\mathrm{o}}, W^{\mathrm{o}}\right),\left\{\left(\varphi_{x}^{\mathrm{o}}, A_{x}^{\mathrm{o}}\right)\right\}_{X \in X^{\mathrm{o}}},\left(\pi^{\mathrm{o}}, N^{\mathrm{o}}\right),\left\{h_{i}^{\mathrm{o}}\right\}_{i \in N^{\mathrm{o}}}\right)$ is an extensive game in the strong sense and $\mathfrak{m}^{\mathrm{o}}=\left(\mathfrak{m}_{1}^{\mathrm{o}}, \ldots, \mathfrak{m}_{n}^{\mathrm{o}}\right)$ is an $n$-tuple of memory functions. Recall that a personal view is given as a pair $\left(\Gamma^{i}, \mathfrak{m}^{i}\right)$, where $\Gamma^{i}=$ $\left(\left(X^{i},<^{i}\right),\left(\lambda^{i}, W^{i}\right),\left\{\left(\varphi_{x}^{i}, A_{x}^{i}\right)\right\}_{X \in X^{i}},\left(\pi^{i}, N^{i}\right), h^{i}\right)$ is an extensive game in the weak sense specifying only the payoff function $h^{i}$ of player $i$ and $\mathrm{m}^{i}$ is a memory function for player $i$ in that game. We assume that player $i$ uses his memory kit $\left(T_{D_{i}}, \mathcal{Y}_{D_{i}}\right)$ in the sense of (3.2) to construct his personal view $\left(\Gamma^{i}, \mathfrak{m}^{i}\right)$.

Strictly speaking, we will not consider the precise process of inductive derivation of a view $\left(\Gamma^{i}, \mathfrak{m}^{i}\right)$. Instead, we consider possible candidates of $\left(\Gamma^{i}, \mathfrak{m}^{i}\right)$ for the result of inductive derivation. For the definition of such a candidate, we need a bridge between $\left(T_{D_{i}}, \mathcal{Y}_{D_{i}}\right)$ and $\left(\Gamma^{i}, \mathrm{~m}^{i}\right)$. We can think of various procedures to have such bridges, but we will use one procedure, called the initial-segment procedure, as stated in Section 4.1. It will become clear shortly why we have chosen this name.

First, for a given candidate $\left(\Gamma^{i}, \mathfrak{m}^{i}\right)$, we define the set $\Theta\left(\Gamma^{i}\right)$ of possible histories in $\Gamma^{i}$ :

$$
\Theta\left(\Gamma^{i}\right)=\left\{\theta^{i}(y): y \in X^{i}\right\}
$$

where $\theta^{i}(y)=\left\langle\left(w_{1}, a_{1}\right), \ldots,\left(w_{m}, a_{m}\right), w_{m+1}\right\rangle$ is the complete history up to $y$ in $\Gamma^{i}$. With the initial-segment procedure, we will connect $\Theta\left(\Gamma^{i}\right)$ with $T_{D_{i}}$.

For the sake of rigor, we make the following definitions. First, a subsequence of $\left[\left(w_{1}, a_{1}\right), \ldots,\left(w_{m}, a_{m}\right)\right]$ is simply defined in the standard manner by regarding each $\left(w_{t}, a_{t}\right)$ as a component of the sequence. Second, $\left\langle\left(w_{1}, a_{1}\right), \ldots,\left(w_{m}, a_{m}\right), w_{m+1}\right\rangle$ is said to be a subsequence of $\left\langle\left(v_{1}, b_{1}\right), \ldots,\left(v_{k}, b_{k}\right), v_{k+1}\right\rangle$ iff $\left[\left(w_{1}, a_{1}\right), \ldots,\left(w_{m}, a_{m}\right),\left(w_{m+1}, a\right)\right]$ is a subsequence of $\left[\left(v_{1}, b_{1}\right), \ldots,\left(v_{k}, b_{k}\right),\left(v_{k+1}, a\right)\right]$ for some $a$. A supersequence is defined in the dual manner. We say that $\left\langle\left(w_{1}, a_{1}\right), \ldots,\left(w_{m}, a_{m}\right), w_{m+1}\right\rangle$ is a maximal sequence in a given set of sequences iff there is no proper supersequence in that set. An initial segment of $\left\langle\left(w_{1}, a_{1}\right), \ldots,\left(w_{m}, a_{m}\right), w_{m+1}\right\rangle$ is a subsequence of the form $\left\langle\left(w_{1}, a_{1}\right), \ldots,\left(w_{k}, a_{k}\right), w_{k+1}\right\rangle$ and $k \leq m$.

Now, we can define the set of initial segments of memory threads in $T_{D_{i}}$ as:

$$
T_{D_{i}}^{*}:=\left\{\langle\xi, w\rangle:\langle\xi, w\rangle \text { is an initial segment of some maximal sequence in } T_{D_{i}}\right\} .
$$

We require $\Theta\left(\Gamma^{i}\right)$ to be the same as $T_{D_{i}}^{*}$ for $\Gamma^{i}$ to be inductively derived from $T_{D_{i}}$. This is why the following is called the initial-segment procedure. A player uses all his initial segments in $T_{D_{i}}$ to construct the histories in $\Gamma^{i}$.

We now give the full set of requirements for an inductively derived personal view based on the initial-segment procedure. As mentioned above, we will give a more general definition of an i.d.view in another paper, which will allow for other inductive procedures (see Section 9.3). In the following definition, we assume that player $i$ is relevant in his own domain $D_{i}$, i.e., $D_{i}$ contains at least one decision node of player $i$.

Definition 4.1 (inductively derived view). A personal view $\left(\Gamma^{i}, \mathfrak{m}^{i}\right)$ for player $i$ is inductively derived from the memory kit $\left(T_{D_{i}}, \mathcal{Y}_{D_{i}}\right)$ iff

P1 (Construction of an extensive game): $\Gamma^{i}$ is an extensive game in the weak sense satisfying:

(a) (Preservation of the informational structure): $\Theta\left(\Gamma^{i}\right)=T_{D_{i}}^{*}$;

(b) (Action sets): $A_{x}^{i}=A_{\lambda^{i}(x)}^{\mathrm{o}}$ for each $x \in X^{i}$;

(c) (Player assignment at decision nodes): $\pi^{i} \cdot \lambda^{i}(x)=\pi^{0} \cdot \lambda^{i}(x)$ for all $x \in X^{i D}$;

(d) (Own payoffs): $h^{i} \cdot \lambda^{i}(x)=h_{i}^{o} \cdot \lambda^{i}(x)$ for each $x \in X^{i E}$; 
Fig. 8. Cane.

P2 (Construction of a memory function): $\mathfrak{m}^{i}$ is a memory function on $X_{i}^{i}=\left\{x \in X^{i}: i \in \pi^{i} \cdot \lambda^{i}(x)\right\}$ satisfying:

(a) (Preservation of memory yarns): $\left\{\mathfrak{m}^{i}(x): x \in X_{i}^{i}\right\} \subseteq \mathcal{Y}_{D_{i}}$;

(b) (Internal consistency): $\theta^{i}(x) \in \mathfrak{m}^{i}(x)$ for any $x \in X_{i}^{i}$;

(c) (Dependence up to observables): if $\theta^{i}(x)=\theta^{i}(y)$, then $\mathfrak{m}^{i}(x)=\mathfrak{m}^{i}(y)$.

We abbreviate an inductively derived view as an i.d.view.

For an i.d.view, the extensive game $\Gamma^{i}$ is constructed based on the set $T_{D_{i}}^{*}$ of initial segments of maximal memory threads in $T_{D_{i}}$. P1a states that the game tree is based on $T_{D_{i}}^{*}$. Conditions P1b, P1c, P1d are the minimum requirements M1, M2, M3 stated in Section 2.1. By P1c and K42, the player set for $\Gamma^{i}$ is determined as

$$
N^{i}=\left\{j \in N^{0}: j \in \pi^{i} \cdot \lambda^{i}(x) \text { for some } x \in X^{i D}\right\} .
$$

Since $\lambda^{i}$ is a surjection from $X^{i}$ to $W^{i}$ by K2, and since $\Theta\left(\Gamma^{i}\right)=T_{D_{i}}^{*}$ by P1a, we have $W^{i} \subseteq W^{o}$. Hence, P1b and P1c are welldefined. For the well-definedness of P1d, it should hold that for any $x \in X^{i E}$, the associated piece $\lambda^{i}(x)$ is an endpiece in the objective game $\Gamma^{\circ}$.

The personal memory function $\mathfrak{m}^{i}$ is constructed based on the set $\mathcal{Y}_{D_{i}}$ of memory yarns. This principle explains condition P2a, while player $i$ is not required to use all of them. Condition P2b states that each yarn $\mathrm{m}^{i}(x)$ should contain the complete history $\theta^{i}(x)$. The reason for this is that $\left(\Gamma^{i}, \mathfrak{m}^{i}\right)$ is now in the mind of player $i$ and can be seen by player $i$ as the objective observer. Still, P2b is one alternative among several possible internal consistency requirements. Condition P2c is more basic, stating that his subjective memory yarns should include no elements additional to what, he believes, have been observed in the play in his view $\Gamma^{i}$.

An analogy with a jigsaw puzzle may help understand the above definition of an i.d.view. Treating the memory threads as the picture on each piece and memory yarns as pieces in a jigsaw puzzle, a player tries to reconstruct an extensive game, though his memory kit may be very incomplete and does not allow him to reach a meaningful view.

To see how an i.d.view is obtained, we look at several examples.

Example 4.1 (Self-scope perfect memory function $\mathrm{m}_{1}^{s p r}$ ). For this memory function, any i.d.view will be a 1-person game played by player 1 , even if the objective game $\left(\Gamma^{0}, \mathrm{~m}^{\mathrm{o}}\right)$ involves multiple players. Consider this memory function on the cane domain described in Example 3.1. The memory kit is given as

$$
T_{D_{1}^{c}\left(\sigma^{\circ}\right)}=\left\{\langle w\rangle,\left\langle(w, a), z_{1}\right\rangle\right\}, \quad \text { and } \quad \mathcal{Y}_{D_{1}^{c}\left(\sigma^{\circ}\right)}=\left\{\{\langle w\rangle\},\left\{\left\langle(w, a), z_{1}\right\rangle\right\}\right\} .
$$

Then $T_{D_{1}^{c}\left(\sigma^{\circ}\right)}=T_{D_{1}^{c}\left(\sigma^{0}\right)}^{*}$, and an i.d.view is given as Fig. 8. It consists of the set of nodes $X^{1}=\left\{y_{0}, y_{1}\right\}, \lambda^{1}\left(y_{0}\right)=w, \lambda^{1}\left(y_{1}\right)=$ $z_{1}, \pi^{1}(w)=\pi^{1}\left(z_{1}\right)=\{1\}, h^{1}\left(z_{1}\right)=2$ and his memory function is given as $\mathfrak{m}^{1}\left(y_{0}\right)=\{\langle w\rangle\}$ and $\mathfrak{m}^{1}\left(y_{1}\right)=\left\{\left\langle(w, a), z_{1}\right\rangle\right\}$. Since $A_{y_{0}}^{1}=A_{w}^{o}=\{a, b\}$ by P1b, condition K33 (bijection requirement) is violated, but K33 ${ }_{0}$ is satisfied.

Now, let us observe that some multiplicity of i.d.views is involved in Definition 4.1, which is caused by the use of hypothetical elements of nodes. In the original game $\left(\Gamma^{\mathrm{o}}, \mathfrak{m}^{0}\right)$ as well as in the derived game $\left(\Gamma^{1}, \mathfrak{m}^{1}\right)$, the nodes are unobservable and auxiliary. We can use different symbols for $y_{0}$ and $y_{1}$ without changing the informational structure of the game. This causes also another type of multiplicity; the game having the duplication of $\left(\Gamma^{1}, \mathfrak{m}^{1}\right)$ described in Fig. 9 satisfies all the requirements of Definition 4.1. We will introduce the concept of a game theoretic $p$-morphism in Section 6 as a means for dealing with those types of multiplicity.

The definition of an inductive derivation based on the initial-segment procedure may not work to serve an i.d.view. Here, we give one negative example and one positive one.

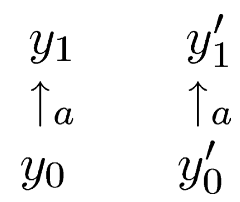

Fig. 9. Duplicated. 


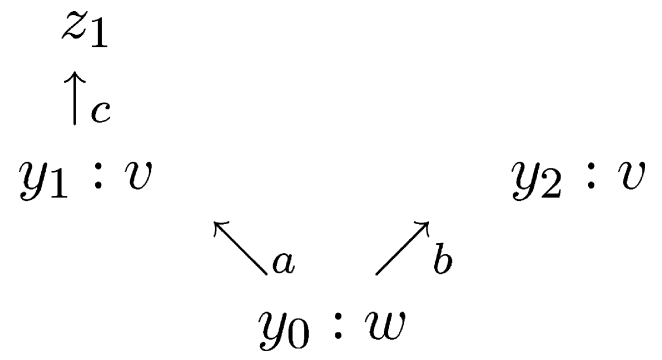

Fig. 10. Failure with $\mathfrak{m}_{1}^{\mathrm{c}}$.

Example 4.2 (Markov memory function $\mathfrak{m}_{i}^{M}:$ general failure). Let player $i$ have the Markov memory function $\mathfrak{m}_{i}^{\mathrm{M}}=\mathfrak{m}_{i}^{o}$. Suppose that player $i$ is relevant in his domain $D_{i}$ in $\Gamma^{\mathrm{o}}$, i.e., $D_{i}$ has at least one decision node $y$. Let $\lambda^{\mathrm{o}}(y)=w$. Since $\mathfrak{m}_{i}^{\mathrm{M}}$ is the Markov memory function, we have $T_{D_{i}}^{*}=T_{D_{i}}=\left\{\langle v\rangle: \lambda^{0}(x)=v\right.$ and $\left.x \in D_{i}\right\}$. This prevents player $i$ from having an i.d.view, since all elements in $T_{D_{i}}^{*}$ have no successors but $\lambda^{\circ}(y)=w$ cannot have a payoff, i.e., P1d cannot be satisfied.

Example 4.3 (Perfect information memory function $\mathfrak{m}_{1}^{P I}:$ full recoverability). Let player 1 have the perfect-information memory function $\mathfrak{m}_{1}^{\mathrm{PI}}$ and let the domain be the full domain $D_{1}^{\mathrm{F}}=X_{1}^{\mathrm{O}}$ in the game of Fig. 6 . In this case, player 1 can reconstruct the objective game $\Gamma^{\circ}$ from his memory kit, except for player 2's payoffs and memory function. This full-recoverability result can be generalized into any game.

When player $i$ has the classical memory function $\mathfrak{m}_{i}^{\mathrm{C}}$ and the full domain $D_{i}^{\mathrm{F}}$, we have also the full-recoverability result. When the domain $D_{i}$ is smaller than $D_{i}^{\mathrm{F}}$, we encounter some difficulty.

Example 4.4 (Classical memory $\mathfrak{m}_{1}^{C}$ with the cane domain: failure). Let player 1 have the classical memory function $\mathfrak{m}_{1}^{C}$ on the cane domain $D_{1}^{c}=D_{1}^{c}\left(\sigma^{\circ}\right)=\left\{x_{0}, z_{1}\right\}$ of (3.4) in Example 3.1. Then $T_{D_{1}^{c}}=\{\langle w\rangle,\langle(w, a), v\rangle,\langle(w, b), v\rangle\}$; one candidate for an i.d.view is described as Fig. 10, which violates conditions $\mathrm{K} 2$ and $\mathrm{K} 31$. Thus, there is no i.d.view in this case.

\section{Direct views}

In Section 4, we gave the definition of an inductively derived view for a given memory kit $\left(T_{D_{i}}, \mathcal{Y}_{D_{i}}\right)$ and found that there may be many i.d.views for each $\left(T_{D_{i}}, \mathcal{Y}_{D_{i}}\right)$. In this section, we single out one of those views which we call the direct view. We will argue that it has a special status among i.d.views or simply among views. Here, we give some results for a direct view to be an i.d.view. In Section 6, we will show that our analysis of direct views is sufficient to describe the game theoretic contents of any i.d.view.

A direct view for a given memory kit $\left(T_{D_{i}}, \mathcal{Y}_{D_{i}}\right)$ is constructed by treating each thread in $T_{D_{i}}^{*}$ as a node in the derived game. As in Section 4, we assume that player $i$ is relevant in his own domain $D_{i}$.

Definition 5.1 (Direct view). A direct view $\left(\Gamma^{d}, \mathfrak{m}^{d}\right)=\left(\left(\left(X^{d},<^{d}\right),\left(\lambda^{d}, W^{d}\right),\left\{\left(\varphi_{x}^{d}, A_{x}^{d}\right)\right\}_{x \in X^{d}},\left(\pi^{d}, N^{d}\right), h^{d}\right), \mathfrak{m}^{d}\right)$ from a memory kit $\left(T_{D_{i}}, \mathcal{Y}_{D_{i}}\right)$ is defined in the following manner:

$\mathrm{d} 1: X^{d}=T_{D_{i}}^{*}$

$\mathrm{d} 2:\langle\xi, v\rangle<^{d}\langle\eta, w\rangle$ iff $\langle\xi, v\rangle$ is a proper initial segment of $\langle\eta, w\rangle$;

d3: (Information function): $\lambda^{d}\langle\xi, v\rangle=v$ for all $\langle\xi, v\rangle \in X^{d}$; and $W^{d}=\left\{v:\langle\xi, v\rangle \in X^{d}\right.$ for some $\left.\xi\right\}$;

d4: (Action sets): $A_{\langle\xi, v\rangle}^{d}=A_{v}^{o}$ for all $\langle\xi, v\rangle \in X^{d}$; and if $\langle\xi, v\rangle \in X^{d D}$, then $\varphi_{\langle\xi, v\rangle}^{d}\langle\xi,(v, a), u\rangle=a$ for each immediate successor $\langle\xi,(v, a), u\rangle$ of $\langle\xi, v\rangle$;

d5: (Player assignment): $\pi^{d}(v)=\pi^{\circ}(v)$ for all $\langle\xi, v\rangle \in X^{d D}$; and $\pi^{d}(v)=N^{d}$ for all $\langle\xi, v\rangle \in X^{d E}$, where $N^{d}=\{j$ : $j \in \pi^{\circ}(v)$ for some $\left.\langle\xi, v\rangle \in X^{d D}\right\}$;

d6: (Payoff function): for any $\langle\xi, v\rangle \in X^{d E}$, if $\lambda^{\circ}(x)=v$ for some $x \in X^{o E}$, then $h^{d}(v)=h_{i}^{o}(v)$; and otherwise, $h^{d}(v)$ is arbitrary;

d7: (Memory function): for any node $\langle\xi, v\rangle$ in $X_{i}^{d}$, if some $\mathfrak{y} \in \mathcal{Y}_{D_{i}}$ contains $\langle\xi, v\rangle$, then $\mathfrak{m}^{d}\langle\xi, v\rangle$ is such $\mathfrak{y} \in \mathcal{Y}_{D_{i}}$; and otherwise, $\mathfrak{m}^{d}\langle\xi, v\rangle=\{\langle\xi, v\rangle\}$.

In the following, $\Gamma^{d}=\left(\left(X^{d},<^{d}\right),\left(\lambda^{d}, W^{d}\right),\left\{\left(\varphi_{x}^{d}, A_{x}^{d}\right)\right\}_{x \in X^{d}},\left(\pi^{d}, N^{d}\right), h^{d}\right)$ defined by $\mathrm{d} 1$ to $\mathrm{d} 6$ is called a direct structure, and $\mathfrak{m}^{d}$ defined by $\mathrm{d} 7$ is a direct memory function.

Condition $\mathrm{d} 6$ has an arbitrariness if some $\langle\xi, v\rangle \in X^{d E}$ does not comes from an endpiece in $\Gamma^{\circ}$. If this is avoided, i.e., a direct structure is an extensive game in the weak sense, it is uniquely determined. Condition d7 may still allow multiple memory functions.

A direct view $\left(\Gamma^{d}, \mathrm{~m}^{d}\right)$ for $\left(T_{D_{i}}, \mathcal{Y}_{D_{i}}\right)$ may not be a personal view; specifically, conditions $\mathrm{K} 2$ and $\mathrm{K} 31$ may be violated. Example 4.4 violates $\mathrm{K} 2$ and $\mathrm{K} 31$, and also, when the objective memory function is the Markov, a direct view 


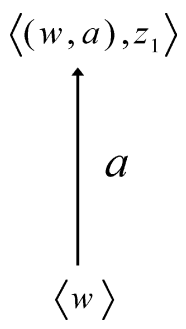

Fig. 11. Unique direct view.

always violates K31. In Theorem 5.1, we will give a condition for a direct view to be a personal view as well as an i.d.view.

Another important comment is about the avoidance of additional hypothetical components such as nodes. It is directly constructed from the components in the memory kit, focusing the initial segments of memory threads in $T_{D_{i}}$. Consequently, the complete history up to each node $x \in X^{d}$ is the same as $x$ itself, which is stated as Lemma 5.1.

Lemma 5.1. For any direct structure $\Gamma^{d}, \theta^{d}(x)=x$ for all $x \in X^{d}$.

Proof. Let $x \in X^{d}$. By d1, $x=\langle\xi, v\rangle=\left\langle\left(w_{1}, a_{1}\right), \ldots,\left(w_{k}, a_{k}\right), v\right\rangle$ is an initial segment of a maximal thread in $T_{D_{i}}$. The path to $\langle\xi, v\rangle$ is $\left\langle w_{1}\right\rangle,\left\langle\left(w_{1}, a_{1}\right), w_{2}\right\rangle, \ldots,\left\langle\left(w_{1}, a_{1}\right), \ldots,\left(w_{k-1}, a_{k-1}\right), w_{k}\right\rangle,\langle\xi, v\rangle$. The complete history up to $\langle\xi, v\rangle$ is the sequence $\left\langle\left(w_{1}, a_{1}\right), \ldots,\left(w_{k-1}, a_{k-1}\right),\left(w_{k}, a_{k}\right), v\right\rangle$, which is $x$ itself.

Let us now look at an example of a direct view.

Example 4.1 (continued): In Figs. 8 and 9, we gave two examples of i.d.views for player 1. This example has a unique direct view, which is given in Fig. 11 and is an i.d.view with the memory function $\mathfrak{m}^{i}(x)=\{x\}$ for all $x \in X^{d}$.

Now, we give conditions for a direct view to be an i.d.view. Recall the assumption that player $i$ is relevant for his own domain $D_{i}$.

Theorem 5.1 (Conditions for a direct view to be I.D.). Let $\left(T_{D_{i}}, \mathcal{Y}_{D_{i}}\right)$ be a memory kit.

(i) The direct structure $\Gamma^{d}$ for $\left(T_{D_{i}}, \mathcal{Y}_{D_{i}}\right)$ is uniquely determined and is an extensive game in the weak sense satisfying P1a-P1d if and only if for any maximal $\langle\xi, v\rangle$ in $T_{D_{i}}^{*}, v=\lambda^{\circ}(x)$ for some $x \in X^{o E}$.

(ii) Let $\Gamma^{d}$ be a direct structure for $T_{D_{i}}$. There is a direct memory function $\mathfrak{m}^{d}$ for $\Gamma^{d}$ satisfying P2a-P2c if and only if for any $\langle\xi, w\rangle \in T_{D_{i}}^{*}$ with $i \in \pi^{\mathrm{O}}(w)$,

there is an $x \in D_{i}$ suchthat $\langle\xi, w\rangle \in \mathfrak{m}_{i}^{\mathrm{o}}(x)$.

This theorem will be proved at the end of this section. The part (i) states that a condition for the unique determination of a direct structure is that every maximal thread in $T_{D_{i}}^{*}$ occurs at an endnode in the objective game. The part (ii) gives a necessary and sufficient condition for a direct memory function prescribed by d7 to satisfy P2a-P2c. When both of these conditions are satisfied, there is a direct view that is i.d., but there is still, however, some arbitrariness in the memory function, which allows for multiple direct views. This is shown by Example 5.1.

Example 5.1. Consider the objective 1-person sequential move game of Fig. 12. Here, the information function is given by $\lambda^{0}\left(y_{t}\right)=v$ for $t=1,2$, and it is the identity function everywhere else. Suppose that the domain of accumulation is the full

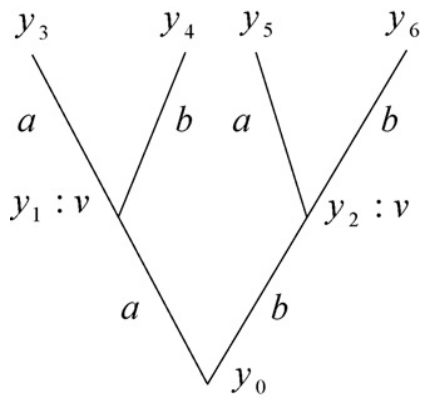

Fig. 12. 1-Person game. 
domain $D_{1}^{\mathrm{F}}=X_{1}^{\mathrm{o}}=X^{\mathrm{o}}$. Let the objective memory function $\hat{\mathrm{m}}_{1}^{\mathrm{o}}$ be defined by

$$
\hat{\mathfrak{m}}_{1}^{\mathrm{o}}\left(y_{t}\right)= \begin{cases}\left\{\theta^{\mathrm{o}}\left(y_{t}\right)\right\} & \text { if } t \neq 1,2 \\ \left\{\left\langle\left(y_{0}, a\right), v\right\rangle,\left\langle\left(y_{0}, b\right), v\right\rangle,\langle v\rangle\right\} & \text { if } t=1 \\ \left\{\left\langle\left(y_{0}, a\right), v\right\rangle,\left\langle\left(y_{0}, b\right), v\right\rangle\right\} & \text { if } t=2\end{cases}
$$

In this example, the direct structure $\Gamma^{d}$ is uniquely determined, which has the same structure as Fig. 12 consisting of nodes $\theta^{\circ}\left(y_{1}\right), \ldots, \theta^{\circ}\left(y_{6}\right)$. However, a memory function has some arbitrariness at the nodes $\theta^{\mathrm{o}}\left(y_{1}\right)$ and $\theta^{\mathrm{o}}\left(y_{2}\right)$. For example, assigning the memory $\mathfrak{m}^{d}\left(\theta^{\mathrm{o}}\left(y_{1}\right)\right)=\mathfrak{m}_{1}^{\mathrm{o}}\left(y_{2}\right)$ and $\mathfrak{m}^{d}\left(\theta^{\mathrm{o}}\left(y_{2}\right)\right)=\mathfrak{m}_{1}^{\mathrm{o}}\left(y_{1}\right)$, together with $\mathfrak{m}^{d}\left(\theta^{\mathrm{o}}\left(y_{t}\right)\right)=\mathfrak{m}_{i}^{\mathrm{o}}\left(y_{t}\right)$ for $t \neq 1$ and $t \neq 2$, gives one i.d.direct view. In this view, the player mixes up his memories at $y_{1}$ and $y_{2}$. In Section 8.2, we will see how this mixing up may create some difficulties. Another view is where he assigns his memory yarns correctly. Still two other views are obtained if he assigns one memory yarn to each of those nodes.

We now introduce two conditions on a memory function, that we will use in combination with Theorem 5.1 to provide a sufficient condition for the uniqueness of a direct view.

(Recall of past memories (RPM)): for all $x, y \in X_{i}^{\mathrm{o}}$, if $\langle\xi, w\rangle \in \mathfrak{m}_{i}^{\mathrm{o}}(x)$ and $x<{ }^{\circ} y$, then $\langle\xi, w\rangle$ is a proper initial segment of some $\langle\eta, v\rangle \in \mathfrak{m}_{i}^{\circ}(y)$.

(Single thread yarns (STY)): $\left|\mathfrak{m}_{i}^{\mathrm{o}}(x)\right|=1$ for all $x \in X_{i}^{\mathrm{o}}$.

The first condition states that every memory thread occurring at a node $x$ of player $i$ will occur as a subsequence of a thread at any later node $y$ of player $i$. This is interpreted as meaning that player $i$ recalls what past memories he had in the current play of the game. The second condition is simply that each yarn consists of a single thread.

The following corollary gives a sufficient condition for the unique determination of a direct view, which guarantees that it is an i.d.view.

Corollary 5.1. Let $D_{i}$ be a closed domain, and let $\left(T_{D_{i}}, \mathcal{Y}_{D_{i}}\right)$ be a memory kit determined by a memory function $\mathfrak{m}_{i}^{\circ}$ satisfying RPM and STY. Furthermore, suppose the latter part, (5.1), of Theorem 5.1.(ii). Then, the direct view $\left(\Gamma^{d}, \mathrm{~m}^{d}\right)$ is uniquely determined by $d 1-d 7$, and $\mathfrak{m}^{d}(x)=\{x\}$ for all $x \in X_{i}^{d}$. Moreover, $\left(\Gamma^{d}, \mathfrak{m}^{d}\right)$ is an i.d.view.

It is straightforward to check that the Self-scope Perfect Recall memory function $\mathrm{m}_{i}^{\mathrm{spr}}$ and the perfect-information memory function $\mathfrak{m}_{i}^{P I}$ on a closed domain satisfy the conditions of Corollary 5.1. Thus, in those cases, we can speak of a unique direct view. We prove this corollary after proving Theorem 5.1 .

Proof of Theorem 5.1. (i) (if): Suppose that for any maximal $\langle\xi, v\rangle$ in $T_{D_{i}}^{*}, v=\lambda^{\mathrm{o}}(x)$ for some $x \in X^{o E}$. Under this supposition, we first show that the direct structure is a uniquely determined extensive game in the weak sense.

Let $\Gamma^{d}$ be a direct structure satisfying d1 to d7. First, observe that the verification of each of K11 to K13 is straightforward by $\mathrm{d} 1, \mathrm{~d} 2$, the non-emptiness of $D_{i}$ and the finite number of threads for each yarn of the memory function $\mathrm{m}_{i}^{\circ}$. Condition $\mathrm{K} 2$ follows from $\mathrm{K} 2$ for $\Gamma^{\mathrm{o}}, \mathrm{d} 1, \mathrm{~d} 2, \mathrm{~d} 3$, condition (2.3) for $\mathrm{m}_{i}^{\mathrm{o}}$, and the supposition of the if part. Condition K31 also follows from the supposition of the if part together with $\mathrm{K} 31$ on $\Gamma^{\mathrm{O}}$ and $\mathrm{d} 4$. Conditions $\mathrm{K} 32$ and $\mathrm{K} 33_{0}$ follow from d1, d2, d3, and d4. K4 uses d5 and d6. Finally, condition $\mathrm{K} 50$ follows from d6. The supposition of the if part implies the payoff function $h_{i}^{d}$ is uniquely determined by d6. Thus, we have shown that the direct structure $\Gamma^{d}$ is determined uniquely as an extensive game in the weak sense.

Next we show that P1a holds. By Lemma 5.1, $\Theta\left(\Gamma^{d}\right)=X^{d}$, and by d1, $X^{d}=T_{D_{i}}^{*}$. Hence, $\Theta\left(\Gamma^{d}\right)=T_{D_{i}}^{*}$. The other parts of P1 follow immediately from the definition of a direct structure.

(Only-if): Suppose that there is a maximal $\langle\xi, v\rangle$ in $T_{D_{i}}^{*}$ and $v=\lambda^{\circ}(x)$ for some $x \in X^{o D}$. By K33 for $\Gamma^{\circ}, A_{x}^{o} \neq \emptyset$. By d4, we have $A_{\langle\xi, v\rangle}^{d}=A_{x}^{o} \neq \emptyset$. However, $\langle\xi, v\rangle \in X^{d E}$ since $\langle\xi, v\rangle$ is maximal in $T_{D_{i}}^{*}$. Hence, K31 is violated for $\Gamma^{d}$, and thus $\Gamma^{d}$ is not an extensive game in the weak sense.

(ii) (If): Suppose that for any $\langle\xi, w\rangle \in T_{D_{i}}^{*}$ with $i \in \pi^{\circ}(w)$, there is an $x \in D_{i}$ such that $\langle\xi, w\rangle \in \mathfrak{m}_{i}^{\circ}(x)$. Then we can define $\mathfrak{m}^{d}\langle\xi, w\rangle=\mathfrak{m}_{i}^{o}(x)$. This is a direct memory function of player $i$ for the direct structure $\Gamma^{d}$, since it associates a memory yarn from $\mathcal{Y}_{D_{i}}$ to each $\langle\xi, w\rangle \in T_{D_{i}}^{*}=X_{i}^{d}$. Then, P2a and P2b are satisfied since by Lemma $5.1, \theta^{d}\langle\xi, w\rangle=\langle\xi$, $w\rangle$. Finally, $\mathfrak{m}^{d}$ satisfies P2c, since by Lemma $5.1, \theta^{d}\langle\xi, w\rangle=\theta^{d}\langle\eta, v\rangle$ implies $\langle\xi, w\rangle=\langle\eta, v\rangle$.

(Only-if): If $\mathfrak{m}^{d}$ is a direct memory function for $\Gamma^{d}$, then the result follows by P2a and P2b for $\mathfrak{m}^{d}$.

Proof of Corollary 5.1. The right-hand side of Theorem 5.1(i) is equivalent to that if $\langle\xi, w\rangle \in T_{D_{i}}^{*}$ and $\lambda^{\circ}(x)=w$ for some decision node $x \in D_{i}$, then $\langle\xi, w\rangle$ is not maximal in $T_{D_{i}}^{*}$. Let $\langle\xi, w\rangle \in T_{D_{i}}^{*}$ and suppose that $\lambda^{\circ}(x)=w$ for some decision node $x \in D_{i}$. Then either $\langle\xi, w\rangle$ is a proper initial segment of some $\langle\eta, v\rangle \in T_{D_{i}}^{*}$, or $\langle\xi, w\rangle \in T_{D_{i}}$. In the first case, $\langle\xi$, w cannot be maximal in $T_{D_{i}}^{*}$. Suppose that $\langle\xi, w\rangle \in T_{D_{i}}$. Then, $\langle\xi, w\rangle \in \mathfrak{m}_{i}^{\circ}\left(x^{\prime}\right)$ for some $x^{\prime} \in D_{i}$. By K2, (2.4), and the supposition that $\lambda^{0}(x)=w$ for some decision node $x \in D_{i}$, it follows that $x^{\prime}$ must also be a decision node in $D_{i}$. Then, by closedness we have a $z \in D_{i}$ with $x^{\prime}<{ }^{0} z$. By $\mathrm{RPM}$, there is a $\langle\eta, v\rangle \in \mathfrak{m}_{i}^{\circ}(z)$ such that $\langle\xi, w\rangle$ is a proper subsequence of $\langle\eta, v\rangle$. Thus, $\langle\xi, w\rangle$ is not maximal in $T_{D_{i}}^{*}$. 
By Theorem 5.1(i), the direct structure $\Gamma^{d}$ is uniquely determined and is an extensive game in the weak sense satisfying P1a-P1d. It remains to be shown that the memory function $\mathfrak{m}^{d}(x)=\{x\}$ is the only memory function for $\Gamma^{d}$ that satisfies P2. By the supposition in the corollary that for any $\langle\xi, w\rangle \in T_{D_{i}}^{*}$ with $i \in \pi^{\circ}(w)$, there is an $x \in D_{i}$, it follows by Theorem 5.1 (ii) that there is a direct memory function for $\Gamma^{d}$ that satisfies P2. By STY, $\mathfrak{m}^{d}(x)=\{x\}$ is the only possible memory function for $\Gamma^{d}$.

\section{Game theoretical p-morphisms: comparisons of views}

In this section, we will show that for any i.d.view $\left(\Gamma^{i}, \mathfrak{m}^{i}\right)$, there is a direct i.d.view $\left(\Gamma^{d}, \mathfrak{m}^{d}\right)$ having the same game theoretical structure. This result reduces the multiplicity of i.d.views, and allows us to concentrate on the direct views for our analysis of i.d.views. For example, the existence of an i.d.view is equivalent to the existence of a direct i.d.view. This consideration will be possible by introducing the concept of a game theoretical $p$-morphism, which is a modification of a p-morphism in the modal logic literature (cf. Ono, 1994; Blackburn et al., 2002). We call it simply a g-morphism.

\subsection{Definition and results}

In the following definition, we abbreviate the superscript $i$ for each component of $\left(\Gamma^{i}, \mathfrak{m}^{i}\right)$ and $\left(\hat{\Gamma}^{i}, \hat{\mathfrak{m}}^{i}\right)$ to avoid unnecessary complications.

Definition 6.1 (Game theoretical p-morphism). Let $(\Gamma, \mathfrak{m})$ and $(\hat{\Gamma}, \hat{\mathfrak{m}})$ be personal views of player $i$. A function $\psi$ from $X$ to $\hat{X}$ is called a g-morphism (game theoretical $p$-morphism) iff

g0: $\psi$ is a surjection from $X$ to $\hat{X}$;

g1: for all $x, y \in X$ and $a \in A_{x}, x<_{a} y$ implies $\psi(x) \hat{<}_{a} \psi(y)$;

g2: for all $\hat{x}, \hat{y} \in \hat{X}, y \in X$ and $a \in \hat{A}_{\hat{x}}, \hat{x} \hat{<}_{a} \hat{y}$ and $\hat{y}=\psi(y)$ imply $x<_{a} y$ and $\hat{x}=\psi(x)$ for some $x \in X$;

g3: (Information pieces): $\hat{\lambda} \cdot \psi(x)=\lambda(x)$ for all $x \in X$;

g4: (Action sets): $\hat{\mathrm{A}}_{\psi(x)}=A_{x}$ for all $x \in X$;

g5: (Player assignment): $\hat{\pi} \cdot \hat{\lambda} \cdot \psi(x)=\pi \cdot \lambda(x)$ for all $x \in X$;

g6: (Payoff function): $\hat{h} \cdot \hat{\lambda} \cdot \psi(x)=h \cdot \lambda(x)$ for all $x \in X^{E}$;

g7: (Memory function): $\hat{\mathfrak{m}} \cdot \psi(x)=\mathfrak{m}(x)$ for all $x \in X_{i}$.

We say that $(\Gamma, \mathfrak{m})$ is $g$-morphic to $(\hat{\Gamma}, \hat{\mathfrak{m}})$, denoted by $(\Gamma, \mathfrak{m}) \longmapsto(\hat{\Gamma}, \hat{\mathfrak{m}})$, iff there is a g-morphism from $(\Gamma, \mathfrak{m})$ to $(\hat{\Gamma}, \hat{\mathfrak{m}})$.

A g-morphism $\psi$ compares one personal view to another one. When a $g$-morphism exists from $(\Gamma, \mathfrak{m})$ and $(\hat{\Gamma}$, $\hat{\mathfrak{m}})$, the set of nodes in $\Gamma$ is mapped onto the set of nodes in $\hat{\Gamma}$, while the game theoretic components of $(\Gamma, \mathfrak{m})$ are preserved. Since $\psi$ is a surjection from $X$ to $\hat{X}$, we cannot take the direct converse of g1, but we take a weak form, g2, which requires that the image $(\hat{\Gamma}, \hat{\mathfrak{m}})$ should not have any additional structure. In sum, the mapping $\psi$ embeds $(\Gamma, \mathfrak{m})$ into $(\hat{\Gamma}, \hat{\mathfrak{m}})$ without losing the game structure. Nevertheless, a g-morphism allows a comparison of quite different games.

In the modal logic literature, the concept of a $p$-morphism is used to compare two Kripke models and their validities. As mathematical objects, Kripke models and extensive games have some similarity in that their basic structures are expressed as some graphs (or trees) (cf., Ono, 1994; Blackburn et al., 2002). In our case, the other game theoretical components including a memory function are placed on the basic tree structure. Therefore, we require our $g$-morphism to preserve those components, i.e., g3-g7. It will be seen that this concept is useful for comparisons of i.d.views for a given memory kit.

Let us consider a few examples to understand $g$-morphisms.

Example 6.1 (Infinite number of P.V.'s g-morphic to a given P.V.). Given a personal view ( $\Gamma$, $m$ ), we can construct a larger personal view by simply replicating $(\Gamma, \mathfrak{m})$. The replicated game with twice as many nodes is $g$-morphic to $(\Gamma, \mathfrak{m})$. By this method, we can construct personal views of any size that are $g$-morphic to $(\Gamma, \mathfrak{m})$. Thus, there are an infinitely many personal views $g$-morphic to $(\Gamma, \mathfrak{m})$.

The following is a less trivial example than the above.

Example 6.2. Fig. 13 gives a g-morphism between two 1-person games, where the memory function for each personal view is assumed to be the perfect-information memory function $\mathfrak{m}^{\mathrm{PI}}$. Define $\psi$ as the identity mapping everywhere except $\psi\left(x_{1}^{\prime}\right)=x_{1}$ and $\psi\left(x_{2}^{\prime}\right)=x_{2}$. This $\psi$ is a $g$-morphism from the left game to the right game.

Here, we give an example where two i.d.views have no g-morphisms. The fact is caused by attached memory functions.

Example 6.3 (Negative example). Consider the objective description of Example 5.1. In this case, the player has four distinct direct views, each of which is an i.d.view. The direct structure is uniquely determined, but there are four possible direct memory functions. No g-morphisms are admitted between each pair of direct views. 


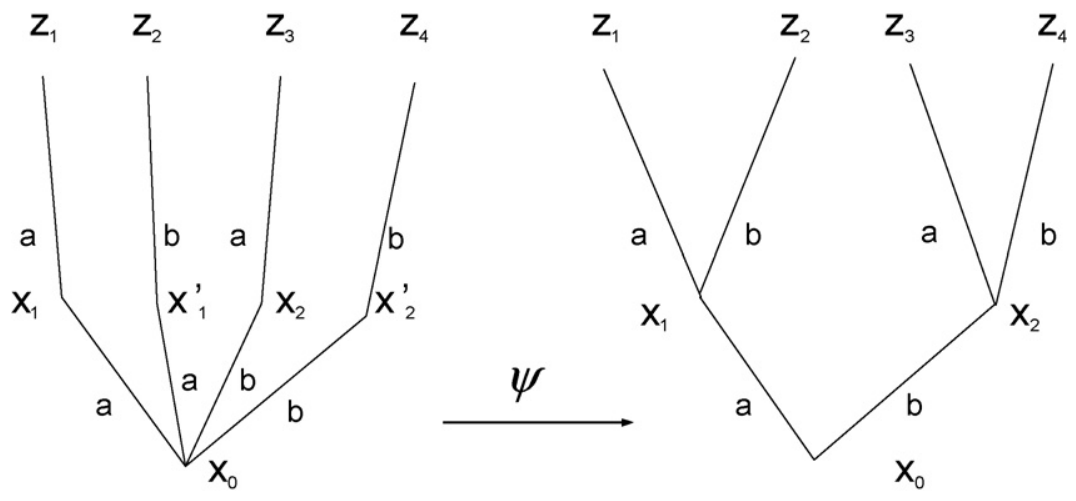

Fig. 13. Less trivial $g$-morphism. 6.2 .

Now, we show that a $g$-morphism fully preserves the i.d.property. All the results presented here will be proved in Section

Theorem 6.1 (Preservation of the I.D. property). Suppose that $(\Gamma, \mathfrak{m})$ is $g$-morphic to $(\hat{\Gamma}, \hat{\mathfrak{m}})$. Then, $(\Gamma, \mathfrak{m})$ is an i.d.view for $\left(T_{D_{i}}, \mathcal{Y}_{D_{i}}\right)$ if and only if $(\hat{\Gamma}, \hat{\mathfrak{m}})$ is an i.d.view for $\left(T_{D_{i}}, \mathcal{Y}_{D_{i}}\right)$.

It follows from this theorem and Example 6.1 that if a given memory kit $\left(T_{D_{i}}, \mathcal{Y}_{D_{i}}\right)$ admits at least one i.d.view, then there are, in fact, an infinite number of i.d.views for $\left(T_{D_{i}}, \mathcal{Y}_{D_{i}}\right)$. Thus, we should consider which i.d.views are more appropriate than others. We will see that the direct views have a special status among the i.d.views. Before that, we give the following simple but basic observations, which can be proved just by looking at the definitions carefully.

Lemma 6.1. (1) The g-morphic relation $\longrightarrow$ satisfies reflexivity and transitivity.

(2) Suppose that $(\Gamma, \mathfrak{m}) \longleftarrow \succ(\hat{\Gamma}, \hat{\mathfrak{m}})$. Then the g-morphism $\psi$ from $(\Gamma, \mathfrak{m})$ to $(\hat{\Gamma}, \hat{\mathfrak{m}})$ satisfies

$\mathrm{g} 0^{*}: \psi$ is a bijection from $X$ to $\hat{X}$;

$\mathrm{g} 1^{*}$ : for all $x, y \in X$ and $a \in A_{x}, x<{ }_{a} y$ if and only if $\psi(x) \hat{<}_{a} \psi(y)$.

By (1), the relation $\longleftrightarrow \succ$ is an equivalence relation over personal views. We can use this relation to consider the equivalence classes of personal views. Any two views in one equivalence class are isomorphic in the sense of $\mathrm{g} 0^{*}, \mathrm{~g} 1^{*}$ and g3-g7, where $\mathrm{g} 2$ is included in $\mathrm{g} 1^{*}$. These two views are identical in our game theoretical sense except for the names of nodes.

In the next theorem we show that every i.d.view is $g$-morphic to a direct view.

Theorem 6.2 (g-Morphism to a direct personal view). Let $\left(T_{D_{i}}, \mathcal{Y}_{D_{i}}\right)$ be a memory kit. For each i.d.view $(\Gamma, \mathfrak{m})$, there is a direct view $\left(\Gamma^{d}, \mathfrak{m}^{d}\right)$ such that $\left(\Gamma^{d}, \mathfrak{m}^{d}\right)$ is a personal view and $(\Gamma, \mathfrak{m})$ is $g$-morphic to $\left(\Gamma^{d}, \mathfrak{m}^{d}\right)$.

The direct view $\left(\Gamma^{d}, \mathfrak{m}^{d}\right)$ given in Theorem 6.2 is also an i.d.view for $\left(T_{D_{i}}, \mathcal{Y}_{D_{i}}\right)$ by Theorem 6.1. This has the implication that we can focus our attention on direct views without loss of generality. The following corollary states that the existence of an i.d.view is characterized by the existence of a direct i.d.view which in turn is characterized by Theorem 5.1.

Corollary 6.1 (Existence of an i.d.view). Let $\left(T_{D_{i}}, \mathcal{Y}_{D_{i}}\right)$ be a memory kit. There is an i.d.view for $\left(T_{D_{i}}, \mathcal{Y}_{D_{i}}\right)$ if and only if there is a direct view that is an i.d.view for $\left(T_{D_{i}}, \mathcal{Y}_{D_{i}}\right)$.

\subsection{Proofs of the results}

First, we start with giving a simple observation.

Lemma 6.2. Let $\psi$ be a g-morphism from $(\Gamma, \mathfrak{m})$ to $(\hat{\Gamma}, \hat{\mathfrak{m}})$. Then $x \in X^{D}$ if and only if $\psi(x) \in \hat{X}^{D}$.

Proof. Let $x \in X^{D}$. Then $x$ has an immediate successor. Thus, $A_{x} \neq \emptyset$ by K33 , which implies $\hat{\mathrm{A}}_{\psi(x)} \neq \emptyset$ by $\mathrm{g} 4$. By K31, $\psi(x) \in \hat{X}^{D}$. The converse follows by tracing back this argument starting with $\psi(x) \in \hat{X}^{D}$.

The next lemma translates $\mathrm{g} 1$ and $\mathrm{g} 2$ into the corresponding $\bar{g} 1$ and $\bar{g} 2$ in terms of the immediate successor relation $<_{a}^{I}$.

Lemma 6.3. Suppose that $\psi$ is a g-morphism from $(\Gamma, \mathfrak{m})$ to $(\hat{\Gamma}, \hat{\mathfrak{m}})$. Then:

$\bar{g} 1$ : for all $x, y \in X$ and $a \in A_{x}, x<{ }_{a}^{I} y$ implies $\psi(x) \hat{<}_{a}^{I} \psi(y)$;

$\bar{g} 2$ : for all $\hat{x}, \hat{y} \in \hat{X}, y \in X$ and $a \in \hat{\mathrm{A}}_{\hat{x}}, \hat{x} \hat{\varkappa}_{a}^{I} \hat{y}$ and $\hat{y}=\psi(y)$ imply $x<{ }_{a}^{I} y$ and $\hat{x}=\psi(x)$ for some $x \in X$. 
Proof. $\bar{g} 1$ : Let $x<_{a}^{I} y$ for some $x, y \in X$. Now, on the contrary, suppose that $\psi(x) \hat{\chi_{a}} \hat{z} \hat{<}_{b} \psi(y)$ for some $\hat{z}$ and $b$. Then, by g2, there is some $z \in X$ such that $\psi(z)=\hat{z}$ and $z<_{b} y$. By K12 for $\Gamma$, we have $x<_{a} z<_{b} y$ or $z<_{b} x<_{a} y$. The first case, $x<<_{a} z<_{b} y$, is impossible since it contradicts $x<_{a}^{I} y$. In the second case, we have $\hat{z} \hat{\chi_{b}} \psi(x)$ by g1, and then, by $\psi(x) \hat{<} a \hat{z}$, we have $\hat{z} \hat{z} \hat{z}$ by the transitivity of K11 for $\hat{\Gamma}$, which contradicts the irreflexivity of K11 for $\hat{\Gamma}$. Thus, we must have $\psi(x) \hat{\varkappa}_{a}^{I} \psi(y)$.

$\bar{g} 2$ : Let $\hat{x} \hat{<}_{a}^{I} \hat{y}$ and $\hat{y}=\psi(y)$ for some $\hat{x}, \hat{y} \in \hat{X}, y \in X$ and $a \in \hat{\mathrm{A}}_{\hat{x}}$. By g2, there is some $x \in X$ such that $x<_{a} y$ and $\hat{x}=\psi(x)$. Now, on the contrary, suppose that $x<_{a} z<_{b} y$ for some $z$ and $b$. Then, by g1, we have $\psi(x) \hat{<}_{a} \psi(z) \hat{<}_{b} \psi(y)$, which is a contradiction to $\hat{x} \hat{<}_{a}^{I} \hat{y}$. Thus, we must have $x<{ }_{a}^{I} y$.

The next lemma makes use of the previous one.

Lemma 6.4. Suppose that $\psi$ is a g-morphism from $(\Gamma, \mathfrak{m})$ to $(\hat{\Gamma}, \hat{\mathfrak{m}})$. Then:

(1) If $\left\langle x_{1}, \ldots, x_{m}\right\rangle$ is a path in $(\Gamma, \mathfrak{m})$, then $\left\langle\psi\left(x_{1}\right), \ldots, \psi\left(x_{m}\right)\right\rangle$ is a path in $(\hat{\Gamma}, \hat{\mathfrak{m}})$ and $\theta\left(x_{t}\right)=\hat{\theta} \cdot \psi\left(x_{t}\right)$ for $t=1, \ldots, m$.

(2) If $\left\langle\hat{x}_{1}, \ldots, \hat{x}_{m}\right\rangle$ is a path in $(\hat{\Gamma}, \hat{\mathfrak{m}})$, then there is a path $\left\langle x_{1}, \ldots, x_{m}\right\rangle$ in $(\Gamma, \mathfrak{m})$ such that $\psi\left(x_{t}\right)=\hat{x}_{t}$ and $\theta\left(x_{t}\right)=\hat{\theta}\left(\hat{x}_{t}\right)$ for $t=1, \ldots, m$.

Proof. (1) Let $\left\langle x_{1}, \ldots, x_{m}\right\rangle$ be a path in $(\Gamma, \mathfrak{m})$. Then there are $a_{1}, \ldots, a_{m-1}$ such that $x_{t}<{ }_{a t}^{I} x_{t+1}$ for $t=1, \ldots, m-1$. Thus, $\psi\left(x_{t}\right) \hat{<}_{a_{t}}^{I} \psi\left(x_{t+1}\right)$ for $t=1, \ldots, m-1$ by $\bar{g} 1$ of Lemma 6.3. This means that $\left\langle\psi\left(x_{1}\right), \ldots, \psi\left(x_{m}\right)\right\rangle$ is a path in $(\hat{\Gamma}, \hat{\mathfrak{m}})$ and, by $\mathrm{g} 3$, $\theta\left(x_{t}\right)=\hat{\theta} \cdot \psi\left(x_{t}\right)$ for $t=1, \ldots, m$.

(2) Let $\left\langle\hat{x}_{1}, \ldots, \hat{x}_{m}\right\rangle$ be a path in $(\hat{\Gamma}, \hat{\mathfrak{m}})$. Then there are $a_{1}, \ldots, a_{m-1}$ such that $\hat{x}_{t} \hat{<}_{a_{t}}^{I} \hat{x}_{t+1}$ for $t=1, \ldots, m-1$. Then, by g0, we can choose an $x_{m} \in X$ with $\psi\left(x_{m}\right)=\hat{x}_{m}$. Then, applying $\bar{g} 2$ of Lemma 6.3 to the last pair $\left(\hat{x}_{m-1}, \hat{x}_{m}\right)$ and $\psi\left(x_{m}\right)=\hat{x}_{m}$, there is an $x_{m-1} \in X$ such that $\psi\left(x_{m-1}\right)=\hat{x}_{m-1}$ and $x_{m-1}<_{a_{m-1}}^{I} x_{m}$. Repeating this argument (exactly speaking, by mathematical induction), we construct $\left\langle x_{1}, \ldots, x_{m}\right\rangle$ with $x_{t}<_{a_{t}}^{I} x_{t+1}$ for $t=1, \ldots, m-1$ and $\psi\left(x_{t}\right)=\hat{x}_{t}$ for $t=1, \ldots, t$. This is a path in $(\Gamma, \mathfrak{m})$ having the required properties.

We have the immediate result from Lemma 6.4 that the mapping $\psi$ preserves the complete histories of information pieces and actions, and the values of the memory yarns.

Lemma 6.5. Suppose that $\psi$ is a g-morphism from $(\Gamma, \mathfrak{m})$ to $(\hat{\Gamma}, \hat{\mathfrak{m}})$. Then:

(a) $\Theta(\Gamma)=\Theta(\hat{\Gamma})$;

(b) $\left\{\mathfrak{m}^{i}(x): x \in X_{i}^{i}\right\}=\left\{\hat{\mathfrak{m}}^{i}(x): x \in \hat{X}_{i}^{i}\right\}$.

Proof. (a) Lemma 6.4(1) states that $\theta(x)=\hat{\theta} \cdot \psi(x)$ for all $x \in X$. Thus, $\Theta(\Gamma) \subseteq \Theta(\hat{\Gamma})$. Conversely, take any $\hat{x} \in \hat{X}$. Lemma 6.4.(2) states that there is an $x$ such that $\theta(x)=\hat{\theta}(\hat{x})$. Thus, $\Theta(\hat{\Gamma}) \subseteq \Theta(\Gamma)$.

(b) By g7, we have $\left\{\mathfrak{m}^{i}(x): x \in X_{i}^{i}\right\} \subseteq\left\{\hat{\mathfrak{m}}^{i}(x): x \in \hat{X}_{i}^{i}\right\}$. The converse inclusion follows from the surjectivity of $\psi$ by g0.

Now, we prove Theorem 6.1. Actually, we prove a more precise claim than the theorem: when there is a $g$-morphism $\psi$ from $(\Gamma, \mathfrak{m})$ to $(\hat{\Gamma}, \hat{\mathfrak{m}})$, each of P1a-P1d and P2a-P2c for $(\Gamma, \mathfrak{m})$ is equivalent to the corresponding one for $(\hat{\Gamma}, \hat{\mathfrak{m}})$.

Proof of Theorem 6.1. Suppose that there is a $g$-morphism $\psi$ from $(\Gamma, \mathfrak{m})$ to $(\hat{\Gamma}, \hat{\mathfrak{m}})$. As stated above, we prove that each requirement of P1a-P1d and $\mathrm{P} 2 \mathrm{a}-\mathrm{P} 2 \mathrm{c}$ for $(\Gamma, \mathfrak{m})$ is equivalent to the corresponding one for $(\hat{\Gamma}, \hat{\mathfrak{m}})$.

P1a: By Lemma 6.5(a), we have $\Theta(\Gamma)=\Theta(\hat{\Gamma})$. P1a holds for $\Gamma$, i.e., $T_{D_{i}}^{*}=\Theta(\Gamma)$, if and only if $T_{D_{i}}^{*}=\Theta(\hat{\Gamma})$, i.e., P1a for $\hat{\Gamma}$. P1b: Let P1b hold for $\Gamma$, i.e., $A_{x}=A_{\lambda(x)}^{\mathrm{o}}$. Consider any $\hat{x} \in \hat{X}$. Then we have some $x \in X$ with $\psi(x)=\hat{x}$. By g4, $\hat{A}_{\hat{x}}=A_{x}$. Thus, $\hat{\mathrm{A}}_{\hat{x}}=A_{\lambda(x)}^{\mathrm{o}}$. Since $\lambda(x)=\hat{\lambda}(\hat{x})$ by g3, we have $\hat{\mathrm{A}}_{\hat{x}}=A_{\hat{\lambda}(\hat{x})}^{\mathrm{o}}$. The converse can be proved similarly.

P1c: Suppose P1c holds for $\hat{\Gamma}$, i.e., $\hat{\pi} \cdot \hat{\lambda}(\hat{x})=\pi^{\mathrm{o}} \cdot \hat{\lambda}(\hat{x})$ for any $\hat{x} \in \hat{X}$. Let $x \in X$. By g3, g5 and P1c for $\hat{\Gamma}$, we have $\pi \cdot \lambda(x)=$ $\hat{\pi} \cdot \hat{\lambda} \cdot \psi(x)=\pi^{\mathrm{o}} \cdot \hat{\lambda} \cdot \psi(x)=\pi^{\mathrm{o}} \cdot \lambda(x)$. Thus, we have P1c for $\Gamma$. The converse is similar.

P1d: Suppose P1d for $\Gamma$. Consider any $\hat{x} \in \hat{X}$. We should show $\hat{h} \cdot \hat{\lambda}(\hat{x})=h_{i}^{o} \cdot \hat{\lambda}(\hat{x})$. By g3,g6 and P1d for $\Gamma$, we have $\hat{h} \cdot \hat{\lambda}(\hat{x})=$ $\hat{h} \cdot \hat{\lambda} \cdot \psi(x)=h \cdot \lambda(x)=h_{i}^{o} \cdot \lambda(x)=h_{i}^{o} \cdot \hat{\lambda} \cdot \psi(x)=h_{i}^{o} \cdot \hat{\lambda}(\hat{x})$. Thus, P1d for $\hat{\Gamma}$. The converse is similar.

P2a: By Lemma 6.5(b), $\left\{\hat{\mathfrak{m}}(\hat{x}): \hat{x} \in \hat{X}_{i}\right\}=\left\{\mathfrak{m}(x): x \in X_{i}\right\}$. Hence, $\mathfrak{m}$ satisfies P2a if and only if $\hat{\mathfrak{m} d o e s .}$

P2b: By g7 and Lemma 6.4, $\mathfrak{m}$ satisfies P2b if and only if $\hat{m} d o e s$.

P2c: Suppose P2c for $\hat{\mathfrak{m}}$. Let $\hat{\theta}(\hat{x})=\hat{\theta}(\hat{y})$. Since $\psi$ is a surjection, we have some $x, y \in X$ such that $\psi(x)=\hat{x}$ and $\psi(y)=\hat{y}$. By Lemma 6.4, $\theta(x)=\hat{\theta}(\hat{x})$ and $\theta(y)=\hat{\theta}(\hat{y})$. Hence $\mathfrak{m}(x)=\mathfrak{m}(y)$ by P2c for $\mathfrak{m}$. Then, by $\mathrm{g} 7, \hat{\mathfrak{m}}(\hat{x})=\mathfrak{m}(x)$ and $\hat{\mathfrak{m}}(\hat{y})=\mathfrak{m}(y)$. Thus, P2c holds for $\hat{\mathfrak{m}}$. The converse is similar.

The next target is to prove Theorem 6.2. We take two steps to have the assertion of the theorem: Under the supposition that $(\Gamma, \mathfrak{m})$ is an i.d.view for memory kit $\left(T_{D_{i}}, \mathcal{Y}_{D_{i}}\right),(1)$ we can find a direct view so that it is a personal view; and $(2)$ it is $g$-morphic to $(\Gamma, \mathfrak{m})$. The first part is given as a lemma, and the second is given as the proof of the theorem. 
Lemma 6.6. Suppose that $(\Gamma, \mathfrak{m})$ is an i.d.view for memory $\operatorname{kit}\left(T_{D_{i}}, \mathcal{Y}_{D_{i}}\right)$. Then $\left(\Gamma^{d}, \mathfrak{m}^{d}\right)$ is a personal view where $\Gamma^{d}$ is the unique direct structure for $\left(T_{D_{i}}, \mathcal{Y}_{D_{i}}\right)$ and $\mathfrak{m}^{d}$ is defined by

$$
\text { for all } x \in X_{i}^{d}, \mathfrak{m}^{d}(x)=\mathfrak{m}\left(y_{x}\right) \text { for some } y_{x} \in X_{i} \text { satisfying } \theta\left(y_{x}\right)=x .
$$

Proof. Let $(\Gamma, \mathfrak{m})$ be an i.d.view for memory kit $\left(T_{D_{i}}, \mathcal{Y}_{D_{i}}\right)$. We first show the right hand side of Theorem 5.1.(i). This implies that $\Gamma^{d}$ is the unique direct structure for $\left(T_{D_{i}}, \mathcal{Y}_{D_{i}}\right)$ and $\Gamma^{d}$ is an extensive game in the weak sense. We next show that (6.1) defines a memory function for $\Gamma^{d}$, from which it follows that $\left(\Gamma^{d}, \mathfrak{m}^{d}\right)$ is personal view.

Suppose, on the contrary, that there is some maximal thread $\langle\xi, v\rangle \in T_{D_{i}}^{*}$ such that $v=\lambda^{\circ}(x)$ for some $x \in X^{o D}$. Then, $A_{v}^{o} \neq \emptyset$ by K33 for $\Gamma^{\circ}$. Since $(\Gamma, \mathfrak{m})$ is an i.d.view for memory kit $\left(T_{D_{i}}, \mathcal{Y}_{D_{i}}\right)$, we have $\Theta(\Gamma)=T_{D_{i}}^{*}$ by P1a. Also, since $\langle\xi, v\rangle$ is maximal in $T_{D}^{*}$, there exists $y \in X^{E}$ such that $\theta(y)=\langle\xi, v\rangle$. Then, by $\mathrm{P} 1 \mathrm{~b}, A_{y}=A_{v}^{0} \neq \emptyset$. This contradicts that $y$ is an endnode in $\Gamma$. Hence, the right hand side of Theorem 5.1.(i) holds.

Now let us see that $\mathfrak{m}^{d}$ is defined by (6.1) is a memory function for $\Gamma^{d}$. By P1a, $W=W^{d}$. Then by c4 and P1b, $\mathfrak{m}^{d}$ is a memory function for $\Gamma^{d}$ since $\mathfrak{m}$ is a memory function for $\Gamma$.

Proof of Theorem 6.2. Let $(\Gamma, \mathfrak{m})$ be an i.d.view for $\left(T_{D_{i}}, \mathcal{Y}_{D_{i}}\right)$. By Lemma $6.9,\left(\Gamma^{d}, \mathfrak{m}^{d}\right)$ is a personal view, where $\Gamma^{d}$ is the unique direct structure for $\left(T_{D_{i}}, \mathcal{Y}_{D_{i}}\right)$ and $\mathfrak{m}^{d}$ is defined by (6.1). First we show that $\left(\Gamma^{d}, \mathfrak{m}^{d}\right)$ is a direct view. Since $\Gamma^{d}$ is the unique direct structure, we need only to show that $\mathfrak{m}^{d}$ satisfies d7. Let $x \in X_{i}^{d}$. By (6.1) and P2b for $\mathfrak{m}, x=\theta\left(y_{x}\right) \in \mathfrak{m}\left(y_{x}\right)=\mathfrak{m}^{d}(x)$ for some $y_{x} \in X_{i}$.

We define the function $\psi$ from $(\Gamma, \mathfrak{m})$ to $\left(\Gamma^{d}, \mathfrak{m}^{d}\right)$ by:

$$
\psi(x)=\theta(x) \text { for all } x \in X .
$$

The proof will be completed if we show that $\psi$ is a $g$-morphism from $(\Gamma, \mathfrak{m})$ to $\left(\Gamma^{d}, \mathfrak{m}^{d}\right)$.

g0: We have $X^{d}=T_{D_{i}}^{*}$ by d1, and also $\Theta(\Gamma)=T_{D_{i}}^{*}$ by P1a for $(\Gamma, \mathfrak{m})$. Thus, $X^{d}=\Theta(\Gamma)$ and so $\psi$ is a surjection from $X$ to $X^{d}$. g1: Let $x<y$. Then, $\theta(x)$ is an initial segment of $\theta(y)$, i.e., $\psi(x)=\theta(x)<^{d} \theta(y)=\psi(y)$ by $\mathrm{d} 2$.

g2: Let $\hat{x}<d \hat{y}$ and $\hat{y}=\psi(y)$. Then, $\hat{x}$ and $\hat{y}$ can be written as $\langle\xi, v\rangle$ and $\langle\eta, w\rangle$ respectively, and by $\mathrm{d} 2,\langle\xi, v\rangle$ is an initial segment of $\langle\eta, w\rangle$. Since $\hat{y}=\psi(y)=\theta(y)=\langle\eta, w\rangle$, and $\langle\xi, v\rangle$ is an initial segment of $\langle\eta, w\rangle$, we can find a unique $x$ on the path to $y$ with $\theta(x)=\langle\xi, v\rangle$. Thus, $x<y$ and $\psi(x)=\theta(x)=\hat{x}$. For g3-g7 we will use the generic history $\theta(x)=\langle\xi, v\rangle$ for the node $x$ in question.

g3: Let $x \in X$. Then $\psi(x)=\theta(x)=\langle\xi, v\rangle$. Hence, $\lambda^{d} \cdot \psi(x)=\lambda^{d}\langle\xi, v\rangle=v$ where the last equality follows from d3. Hence, we have shown that $\lambda^{d} \cdot \psi(x)=\lambda(x)$.

g4: Let $x \in X$. Then, by d4, $A_{\langle\xi, v\rangle}^{c}=A_{v}^{\mathrm{o}}$. By P1b, we have $A_{x}=A_{\lambda(x)}^{\mathrm{o}}=A_{v}^{\mathrm{o}}$. Hence, $A_{\psi(x)}^{c}=A_{x}$.

g5: Let $x \in X$. By d3, $\pi^{d} \cdot \lambda^{d} \cdot \psi(x)=\pi^{c}(v)$. If $x \in X^{D}$, then by P1c, $\pi \cdot \lambda(x)=\pi^{0} \cdot \lambda(x)=\pi^{0}(v)$. Also, since $x \in X^{D}$, it follows by Lemma 6.1 that $\langle\xi, v\rangle \in X^{d D}$. Hence, by d5, $\pi^{d}(v)=\pi^{\mathrm{o}}(v)$. Thus, for $x \in X^{D}$ we have the desired result that $\pi^{d} \cdot \lambda^{d} \cdot \psi(x)=$ $\pi \cdot \lambda(x)$. Next consider $x \in X^{E}$. Then by K42, $\pi \cdot \lambda(x)=\left\{j: j \in \pi \cdot \lambda(y)\right.$ for some $\left.y \in X^{D}\right\}$. By Lemma $6.2, \mathrm{~g} 0$ and d5, it follows that this set is equivalent to $\pi^{d}(v)$.

g6: Let $x \in X^{E}$. By P1a and P1d, $v=\lambda(x)=\lambda^{\circ}(y)$ for some $y \in X^{o E}$, and $h(v)=h_{i}^{\circ}(v)$. By Lemma 6.1 and $\mathrm{g} 3, \psi(x) \in X^{d E}$ and $\lambda \cdot \psi(x)=v=\lambda^{o}(y)$ for some $y \in X^{o E}$. So, by d6, $h^{d}(v)=h_{i}^{o}(v)$. Hence, we have shown that $h^{d} \cdot \lambda^{d} \cdot \psi(x)=h \cdot \lambda(x)$.

g7: Let $x \in X_{i}$. Then by the definition of $\psi$, (6.1) and P2c for $\mathfrak{m}$, it follows that $\mathfrak{m}^{d} \cdot \psi(x)=\mathfrak{m}^{d} \cdot \theta(x)=\mathfrak{m}(y)=\mathfrak{m}(x)$.

\section{Decision making and prescribed behavior in inductive game theory}

The inductive derivation of an individual view from past experiences is not the end of the entire scenario of our theory. The next step is to use an i.d.view for decision making and to bring the prescribed (or modified) behavior back to the objective situation. This is the third stage of Fig. 1. Because this paper aims to present a basic and entire scenario of our theory, we will here concentrate on a clear-cut case. Specifically, we assume in this and next sections that the objective memory function $\mathfrak{m}_{i}^{\mathrm{o}}$ for each player $i$ is given as the self-scope perfect memory function $\mathfrak{m}_{i}^{\text {spr }}$, and that player $i$ has the active domain $D_{i}^{\mathrm{A}}\left(\sigma^{\mathrm{o}}\right)$. Then, we will discuss how he can use the inductively derived view for his decision making as well as how the prescribed behavior helps his objective behavior. This gives an experiential foundation for Nash equilibrium.

\subsection{Decision making using a personal view}

Fig. 14 describes the steps from experimentation (trial and error) to decision making using an i.d.view. One basic question is whether the i.d.view helps the player for his decision making, as well as whether the decision can be used in the objective situation when he brings it back there. In this and next sections, we will discuss these questions.

We assume that each player $i$ : 


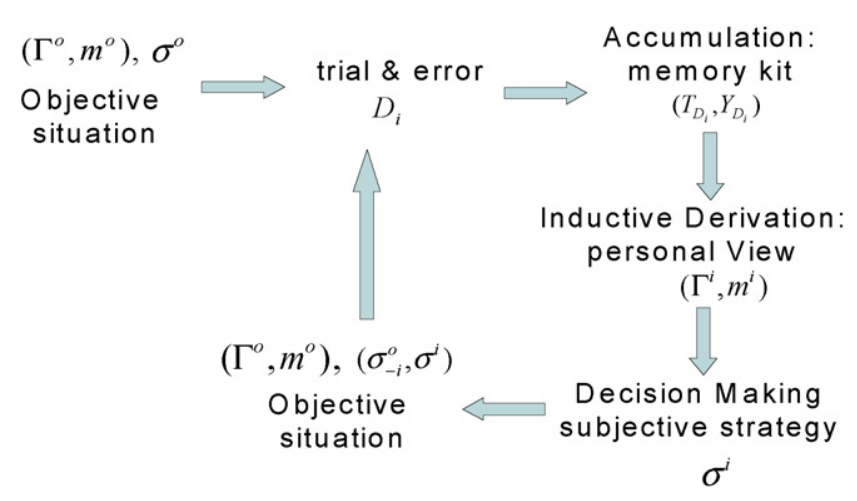

Fig. 14. Various phases.

(7a) is relevant in his own domain;

(7b) has the self-scope perfect recall memory function $m_{i}^{\mathrm{o}}=m_{i}^{\mathrm{spr}}$;

(7c) follows a behavior pattern $\sigma_{i}^{0}$;

(7d) accumulates memories over his active domain $D_{i}^{\mathrm{A}}\left(\sigma^{\circ}\right)$;

(7e) adopts the direct view $\left(\Gamma^{d}, m^{d}\right)$.

Under these assumptions, it is already proved in Corollary 5.1 that there is a unique direct i.d.view for each player $i$. Now, we consider the case where player $i$ adopts this direct i.d.view $\left(\Gamma^{d}, m^{d}\right)$. Nevertheless, the direct structure $\Gamma^{d}$ may not be an extensive game in the strong sense, which may create some complications in the following discourse. Thus, we make the following assumption to avoid it: for each player $i$,

(7f) for all $x, y \in D_{i}^{A} \cap X^{o E}, \theta^{\circ}(x)_{i}$ is not a proper subsequence of $\theta^{\circ}(y)_{i}$.

Under this assumption, the direct view $\left(\Gamma^{d}, m^{d}\right)$ is an extensive game in the strong sense, which will be stated in Lemma 7.1.

Condition $7 \mathrm{f}$ is implied by Kuhn's (1953) condition that each information piece for player $i$ occurs at most once in each play in $\Gamma^{\mathrm{o}}$, which was actually stated in terms of information sets in Kuhn (1953). Fig. 15 A, called the absent-minded driver game in Piccione and Rubinstein (1997), with the self-scope perfect-recall function $\mathfrak{m}_{i}^{\text {spr }}$ violates Condition 7f. In this case, $\langle(E, e), 1\rangle$ belongs to $T_{D_{1}}$, but not to $T_{D_{1}}^{*}$ since $\langle(E, c),(E, e), 1\rangle$ is a proper supersequence of $\langle(E, e), 1\rangle$. Fig. $15 \mathrm{~B}$ is the direct view but is not an extensive game in the strong sense.

The proofs of the results will be given in the end of this subsection.

Lemma 7.1. The direct view $\left(\Gamma^{d}, \mathfrak{m}^{d}\right)$ for $\left(T_{D_{i}}, \mathcal{Y}_{D_{i}}\right)=\left(T_{D_{i}^{A}\left(\sigma^{\circ}\right)}, \mathcal{Y}_{D A\left(\sigma^{\circ}\right)}\right)$ is uniquely determined and is an i.d.view satisfying:

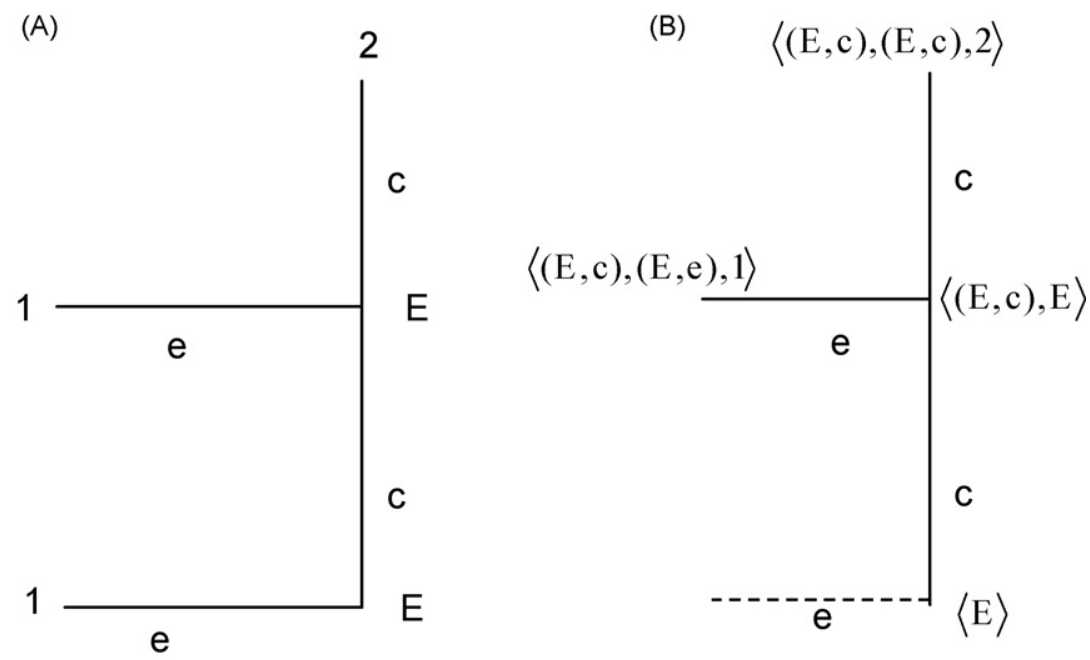

Fig. 15. Violation of condition $7 \mathrm{f}$ and the direct view. 
(a) $\Gamma^{d}$ is a 1-person extensive game in the strong sense with $N^{d}=\{i\}$;

(b) $\mathfrak{m}^{d}$ satisfies P2a with equality, i.e., $\left\{\mathfrak{m}^{d}(x): x \in X^{d}\right\}=\mathcal{Y}_{D_{i}^{A}\left(\sigma^{\circ}\right)}$.

For the consideration of utility maximization of a behavior pattern $\sigma_{i}$, player $i$ needs to consider the sets of compatible endnodes for various behavior patterns. Recall from (2.15) that $\lambda(\sigma)$ denotes the set of compatible endpieces for a profile of behavior patterns $\sigma=\left(\sigma_{1}, \ldots, \sigma_{n}\right)$. Since $\Gamma^{d}$ is a 1-person extensive game in the strong sense, the set of compatible endpieces will be a singleton set for each behavior pattern $\sigma_{i}$ of player $i$. Consequently, we will use $\lambda^{d}\left(\sigma_{i}\right)$ here to denote the compatible endpiece in $\Gamma^{d}$ for $\sigma_{i}$.

Then, player $i$ has a subjective strategy $\sigma_{i}^{d}$ in $\Gamma^{d}$ to maximize $h^{d}$ in the following sense:

$$
h^{d} \cdot \lambda^{d}\left(\sigma_{i}^{d}\right) \geq h^{d} \cdot \lambda^{d}\left(\sigma_{i}\right) \text { for all } \sigma_{i} \in \Sigma_{i}^{d} .
$$

Once again, we emphasize that this decision is made in the personal view $\left(\Gamma^{d}, \mathfrak{m}^{d}\right)$ of player $i$, i.e., in the mind of player $i$. This conceptually differs from the payoff maximization in the objective situation, which is now the subject to be considered.

After the choice of the subjective strategy in (7.1), player $i$ brings back $\sigma_{i}^{d}$ to the objective situation $\left(\Gamma^{\mathrm{o}}, \mathrm{m}^{\mathrm{o}}\right)$, adjusting his behavior pattern $\sigma_{i}^{o}$ with $\sigma_{i}^{d}$. The adjustment from his objective behavior $\sigma_{i}^{o}$ into $\sigma_{i}^{1}$ is as follows: for all $x \in X_{i}^{o}$,

$$
\sigma_{i}^{1}(x)= \begin{cases}\sigma_{i}^{d}\langle\xi, v\rangle & \text { if } \mathfrak{m}_{i}^{\mathrm{o}}(x)(=\{\langle\xi, v\rangle\}) \in \mathcal{Y}_{D_{i}^{A}\left(\sigma^{\circ}\right)} \\ \sigma_{i}^{\mathrm{o}}(x) & \text { if } \mathfrak{m}_{i}^{\mathrm{o}}(x) \notin \mathcal{Y}_{D_{i}^{\mathrm{A}}\left(\sigma^{\circ}\right)} .\end{cases}
$$

That is, player $i$ follows $\sigma_{i}^{d}$ whenever a memory yarn in $\mathcal{Y}_{D_{i}^{A}\left(\sigma^{\circ}\right)}$ occurs; and otherwise, he keeps the old behavior pattern. This adjustment produces a behavior pattern for player $i$ in $\Gamma^{\mathrm{o}}$, i.e., $\sigma_{i}^{1} \in \Sigma_{i}^{\mathrm{o}}$. The next theorem states that the modified strategy $\sigma_{i}^{1}$ of player $i$ defined by (7.2) is actually objectively utility maximizing for player $i$ in $\Gamma^{\mathrm{o}}$ when the other players follow their regular behavior $\sigma_{-i}^{\mathrm{o}}$ in $\Gamma^{\mathrm{o}}$.

Before the next theorem, we give a small remark. Since the objective game $\Gamma^{\mathrm{o}}$ is also an extensive game in the strong sense, the set of compatible endpieces $\lambda^{\mathrm{o}}\left(\sigma_{i}, \sigma_{-i}^{\mathrm{o}}\right)$ will also be a singleton for player $i$ 's behavior pattern $\sigma_{i}$ and the other players' behavior patterns $\sigma_{-i}^{0}$. We follow the convention of using $\lambda^{0}\left(\sigma_{i}, \sigma_{-i}^{0}\right)$ to denote the compatible endpiece, not the set of compatible endpieces.

Theorem 7.1 (One-person utility maximization in the $n$-person game). The strategy $\sigma_{i}^{1}$ defined by (7.2) satisfies the objective payoff maximization for player i, i.e.,

$$
h_{i}^{\mathrm{o}} \cdot \lambda^{\mathrm{o}}\left(\sigma_{i}^{1}, \sigma_{-i}^{\mathrm{o}}\right) \geq h_{i}^{\mathrm{o}} \cdot \lambda^{\mathrm{o}}\left(\sigma_{i}, \sigma_{-i}^{\mathrm{o}}\right) \text { for all } \sigma_{i} \in \Sigma_{i}^{\mathrm{o}} .
$$

We emphasize that this is not the utility maximization obtained directly in the objective situation. Instead, the utility maximization is made in his i.d.view $\left(\Gamma^{d}, \mathfrak{m}^{d}\right)$, and then the modified strategy $\sigma_{i}^{1}$ is brought to the objective situation $\left(\Gamma^{\mathrm{o}}, \mathrm{m}^{\mathrm{o}}\right)$. It happens that it actually maximizes his objective utility function. This process of obtaining the objective utility maximization occurs only after many repetitions of collecting data to construct his view.

Thus, we have succeeded in having individual utility maximization in the well-defined form in both subjective and objective senses. Nevertheless, once we leave the case of 7a-7f, player $i$ would have many difficulties at various steps in Fig. 14. These problems will be discussed in Section 8.2 and in separate papers.

Proof of Lemma 7.1. (a) The condition $N^{d}=\{i\}$ follows immediately since $\mathfrak{m}_{i}^{o}=\mathfrak{m}_{i}^{\text {spr }}$. By Corollary 5.1, it suffices to show that $\Gamma^{d}$ satisfies K14 and K33.

K14: Since $\Gamma^{\mathrm{o}}$ is an extensive game in the strong sense, each strategy combination determines a unique play. Let $\left\langle x_{1}, \ldots, x_{m}\right\rangle$ be the unique play determined by $\sigma^{0}$, and let $x_{t}$ be the first node of player $i$ in this play, i.e., $i \in \pi^{0} \cdot \lambda^{0}\left(x_{t}\right)$ and $i \notin \pi^{\mathrm{o}} \cdot \lambda^{\mathrm{o}}\left(x_{s}\right)$ for all $s<t$. Then $\theta^{\mathrm{o}}\left(x_{t}\right)=\left\langle\left(\lambda^{\mathrm{o}}\left(x_{1}\right), \sigma_{j_{1}}^{\mathrm{o}}\left(x_{1}\right)\right), \ldots,\left(\lambda^{\mathrm{o}}\left(x_{t-1}\right), \sigma_{j_{t-1}}^{\mathrm{o}}\left(x_{t-1}\right)\right), \lambda^{\mathrm{o}}\left(x_{t}\right)\right\rangle$ where $j_{1}, \ldots, j_{t-1}$ denote the players moving at $x_{1}, \ldots, x_{t-1}$, respectively. Let $\left(\sigma_{i}, \sigma_{-i}^{o}\right)$ be any other strategy combination where all the players other than player $i$ choose according to $\sigma^{0}$. Then, the first $t$ nodes in the play determined by this strategy combination must also be $x_{1}, \ldots, x_{t}$. Hence, for any play determined on the active domain, $x_{t}$ is the first node of player $i$. Thus, $x_{t}$ determines the smallest node $\theta^{\mathrm{o}}\left(x_{t}\right)$ in $X^{d}$.

K33: We show that for each $\langle\xi, v\rangle \in X^{d D}$, the function $\varphi_{\langle\xi, v\rangle}^{d}$ defined in $\mathrm{d} 4$ is a bijection. Let $\langle\xi, v\rangle \in X^{d D}$ and let $a$ be an arbitrary action in $A_{\langle\xi, v\rangle}$. Since $\langle\xi, v\rangle \in X^{d D}$ and the memory function is $\mathrm{m}_{i}^{\mathrm{spr}}$, we have $\langle\xi, v\rangle=\theta^{\mathrm{o}}(x)_{i}$ for some $x \in X_{i}^{\mathrm{o}}$, and $x$ is on the path determined by some $\left(\sigma_{i}, \sigma_{-i}^{o}\right)$. Consider the strategy $\sigma_{i}^{\prime}$ defined by

$$
\sigma_{i}^{\prime}(y)= \begin{cases}\sigma_{i}(y) & \text { if } \mathfrak{m}_{i}^{\mathrm{o}}(y) \neq \mathfrak{m}_{i}^{\mathrm{o}}(x) \\ a & \text { if } \mathfrak{m}_{i}^{\mathrm{o}}(y)=\mathfrak{m}_{i}^{\mathrm{o}}(x) .\end{cases}
$$

Since $\mathfrak{m}_{i}^{o}=\mathfrak{m}_{i}^{\mathrm{spr}}$, it follows that $\mathfrak{m}_{i}^{\mathrm{o}}(y) \neq \mathfrak{m}_{i}^{\mathrm{o}}(x)$ for any $y \in X_{i}^{o D}$ with $y<{ }^{o} x$. Hence $x$ is on the play determined by $\left(\sigma_{i}^{\prime}, \sigma_{-i}^{\mathrm{o}}\right)$. Since the other players follow their strategies in $\sigma^{0}$, the action $a$ determines a unique immediate successor $x^{\prime}$ of $x$ with $\mathfrak{m}_{i}^{\text {spr }}\left(x^{\prime}\right)=\{\langle\xi,(v, a), u\rangle\}$. Then we find also an endnode $z$ coming from $x^{\prime}$. Then, $\langle\xi,(v, a), u\rangle$ is an initial segment of $\theta_{i}^{\mathrm{o}}(z)$. By condition $7 \mathrm{f}, \theta_{i}^{\mathrm{o}}(z)$ is a maximal sequence in $T_{D_{i}}$. These mean that $\langle\xi,(v, a), u\rangle \in T_{D_{i}}^{*}=X^{d}$. 
We can show similarly that a different action $a^{\prime} \in A_{\langle\xi, v\rangle}$ determines a different immediate successor $\left\langle\xi,\left(v, a^{\prime}\right), u^{\prime}\right\rangle \in X^{d}$, so the mapping $\varphi_{\langle\xi, v\rangle}^{d}$ from $\langle\xi,(v, a), u\rangle$ to $a$ is a bijection.

(b) Let $x \in D_{i}$. We show that $\mathfrak{m}_{i}^{o}(x) \in\left\{\mathfrak{m}^{d}(y): y \in X^{d}\right\}$. Since $\mathfrak{m}_{i}^{o}=\mathfrak{m}_{i}^{\text {spr }}$, we have $T_{D_{i}}=T_{D_{i}}^{*}$. Since $\mathfrak{m}_{i}^{o}(x)=\left\{\theta^{o}(x)_{i}\right\}$, it follows that $\theta^{\circ}(x)_{i} \in T_{D_{i}}^{*}=X^{d}$. Corollary 5.1 states that the direct view $\left(\Gamma^{d}, \mathfrak{m}^{d}\right)$ exists uniquely and $\mathfrak{m}^{d}(y)=\{y\}$ for all $y \in X_{i}^{d}$. Hence, $\mathfrak{m}^{d}\left(\theta^{\mathrm{o}}(x)_{i}\right)=\left\{\theta^{\mathrm{o}}(x)_{i}\right\}=\mathfrak{m}_{i}^{\mathrm{o}}(x)$.

Proof of Theorem 7.1. Consider any $\sigma_{i} \in \Sigma_{i}^{0}$. Recall that the endnode determined by $\left(\sigma_{i}, \sigma_{-i}^{0}\right)$ in $\Gamma^{0}$ is denoted by $z\left(\sigma_{i}, \sigma_{-i}^{0}\right)$. Let $x=z\left(\sigma_{i}, \sigma_{-i}^{0}\right)$. Consider the history of player $i$ at $x$, i.e., $\theta^{\mathrm{o}}(x)_{i}=\left\langle\left(w_{1}, a_{1}\right), \ldots,\left(w_{m}, a_{m}\right), w_{m+1}\right\rangle$ with $w_{m+1}=\lambda^{\mathrm{o}}(x)$, and also, let the corresponding history of nodes be given as $\left\langle x_{1}, \ldots, x_{m}, x_{m+1}\right\rangle$ with $x_{m+1}=x$. Then, $\lambda^{0}\left(x_{t}\right)=w_{t}$ and $\sigma_{i}\left(x_{t}\right)=a_{t}$ for all $t=1, \ldots, m$. Hence, we choose a strategy $\tau_{i}^{d}$ having the property that $\tau_{i}^{d}\left\langle\left(w_{1}, a_{1}\right), \ldots,\left(w_{t-1}, a_{t-1}\right), w_{t}\right\rangle=\sigma_{i}\left(x_{t}\right)$ for $t=1, \ldots, m$. Then, the compatible endpiece $\lambda^{0}\left(\sigma_{i}, \sigma_{-i}^{0}\right)=\{v\}$ is the same as $\lambda^{d}\left(\tau_{i}^{d}\right)$. Hence, $\lambda^{o}\left(\sigma_{i}^{1}, \sigma_{-i}^{o}\right)=\lambda^{d}\left(\tau_{i}^{d}\right)$. If we apply this procedure to $\sigma_{i}^{1}$, then we have $\sigma_{i}^{d}$ satisfying (7.1). Hence, we have $\lambda^{0}\left(\sigma_{i}^{1}, \sigma_{-i}^{0}\right)=\lambda^{d}\left(\sigma_{i}^{1}\right)$.

By $\mathrm{d} 7$ and using the above result, we have $h_{i}^{\mathrm{o}} \cdot \lambda^{\mathrm{o}}\left(\sigma_{i}^{1}, \sigma_{-i}^{0}\right)=h^{d} \cdot \lambda^{d}\left(\sigma_{i}^{d}\right) \geq h^{d} \cdot \lambda^{d}\left(\tau_{i}^{d}\right)=h_{i}^{0} \cdot \lambda^{0}\left(\sigma_{i}, \sigma_{-i}^{0}\right)$.

\subsection{An experiential foundation for Nash equilibrium}

It is straightforward to extend Theorem 7.1 to all players relevant in their own domains and to obtain a Nash equilibrium. Here, we still state this theorem, since it gives one explanation of Nash equilibrium from the experiential viewpoint. For it, however, we need some more notation and one more definition.

First, since our discussion involves more than one i.d.view, we put subscript " $i$ " to the direct i.d.view of player $i$, i.e., $\left(\Gamma_{i}^{d}, \mathfrak{m}_{i}^{d}\right)$. Second, for each player $i$ who is relevant in his own domain, we define the induced strategy $\sigma_{i}^{d}$ of $\sigma^{\circ}$ to the direct i.d.view $\left(\Gamma_{i}^{d}, \mathfrak{m}_{i}^{d}\right)$ for $\left(T_{D_{i}^{A}\left(\sigma^{\circ}\right)}, \mathcal{Y}_{D^{A}\left(\sigma^{0}\right)}\right)$ by: for all $\langle\xi, w\rangle \in X_{i}^{d}$,

$$
\sigma_{i}^{d}\langle\xi, w\rangle=\sigma_{i}^{o}(x) \text { for any } x \in X_{i}^{o} \text { with } \theta_{i}^{o}(x)=\langle\xi, w\rangle \text {. }
$$

The well-definedness of (7.4) is verified as follows. First, by the properties of the SPR memory function, for each $\langle\xi, w\rangle \in X_{i}^{d}$, there is an $x \in X_{i}^{\mathrm{o}}$ such that $\theta_{i}^{\mathrm{o}}(x)=\langle\xi, w\rangle$. Secondly, since $\theta_{i}^{\mathrm{o}}(x)=\theta_{i}^{\mathrm{o}}(y)$ implies $\mathfrak{m}_{i}^{\mathrm{spr}}(x)=\mathfrak{m}_{i}^{\mathrm{spr}}(y)$, the strategy defined by (7.4) does not depend upon the choice of $x$. Finally, we verify (1) and (2) for $\sigma_{i}^{d}$. The condition (1) follows from d4. Condition (2) is also satisfied since by Corollary 5.1, the direct memory function of player $i$ is uniquely determined as $\mathfrak{m}_{i}^{d}\langle\xi, w\rangle=\{\langle\xi, w\rangle\}$.

Then we have the following theorem, which is a straightforward implication of Theorem 7.1.

Theorem 7.2 (Experimental foundation for Nash equilibrium). A profile $\sigma^{\circ}$ of behavior patterns is a Nash equilibrium in $\left(\Gamma^{\mathrm{o}}, \mathrm{m}^{\mathrm{o}}\right)$ if and only if for each player $i \in N^{\mathrm{O}}$ who is relevant in his domain $D_{i}^{\mathrm{A}}\left(\sigma^{\mathrm{o}}\right)$, the induced strategy $\sigma_{i}^{d}$ of $\sigma^{\mathrm{o}}$ to the direct view $\left(\Gamma_{i}^{d}, \mathfrak{m}_{i}^{d}\right)$ for the memory kit $\left(T_{D_{i}^{\mathrm{A}}\left(\sigma^{0}\right)}, \mathcal{Y}_{D_{i}^{\mathrm{A}}\left(\sigma^{0}\right)}\right)$ satisfies condition (7.1).

Recall that we have adopted the assumptions 7a-7f. Under these assumptions, each player makes his decision in his 1-person derived view. The theorem states that the behavior pattern $\sigma^{0}$ is a Nash equilibrium in the objective game $\left(\Gamma^{\mathrm{o}}, \mathrm{m}^{\mathrm{o}}\right)$ if and only the induced strategy for each player $i$ maximizes his utility in the direct view $\left(\Gamma_{i}^{d}, \mathfrak{m}_{i}^{d}\right)$. Thus, this theorem decomposes the Nash equilibrium in $\left(\Gamma^{\mathrm{o}}, \mathfrak{m}^{\mathrm{o}}\right)$ into utility maximizations in $n$ one-person games.

As discussed in Section 3, the accumulation of $\left(T_{D_{i}^{\mathrm{A}}\left(\sigma^{\circ}\right)}, \mathcal{Y}_{D_{i}^{\mathrm{A}}\left(\sigma^{\circ}\right)}\right)$ and the inductive derivation of $\left(\Gamma_{i}^{d}, \mathrm{~m}_{i}^{d}\right)$ need many repetitions of the game $\left(\Gamma^{\mathrm{o}}, \mathrm{m}^{\mathrm{o}}\right)$. Also, in the present scenario, each player revises his behavior over $D_{i}^{\mathrm{A}}\left(\sigma^{\mathrm{o}}\right)$, and other players may be influenced by his revision, and may change their personal views. This process of changing may continue. The above theorem describes a stationary state in this revision process.

The revision process may take a long time to reach a Nash equilibrium or even may not reach a Nash equilibrium. Furthermore, we did not explicitly consider the case where the players' trials and errors are restricted. If we take these limitations over experimentations, the above "Nash equilibrium" is understood as a Nash equilibrium relative to the restricted domains of actions.

In the above senses, Theorem 7.2 is one characterization of Nash equilibrium from the experiential viewpoint. In separate papers, we will discuss other characterizations of Nash equilibrium and/or difficulties arising for them. Finally, we give one example to suggest the nonconvergence of the process of revising behavior via constructed personal views. If the objective game $\left(\Gamma^{0}, \mathfrak{m}^{0}\right)$ has no Nash equilibria, then the above process does not converge. The following example has a Nash equilibrium.

Example 7.1 (Nonconvergence). Consider the 2-person simultaneous game which is described as Fig. 16 and its payoffs are given in Fig. 17. The bold arrow is the regular path $\left(\boldsymbol{s}_{12}, \boldsymbol{s}_{22}\right)$ and each player is presumed to have the SPR memory function.

Player 1's direct i.d.view is the 1-person game summarized by the matrix form of Fig. 18, and player 2's i.d.view is the 1-person game summarized in Fig. 19.

In this case, player 1 maximizes his utility in his i.d.view by choosing $\boldsymbol{s}_{12}$. Thus, he has no incentive to change his objective behavior from the regular pattern. However, player 2 maximizes his utility in his i.d.view by changing from $\boldsymbol{s}_{22}$ to $\boldsymbol{s}_{23}$. 


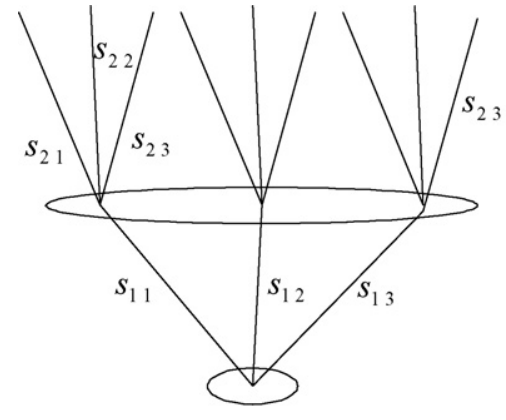

the entire game

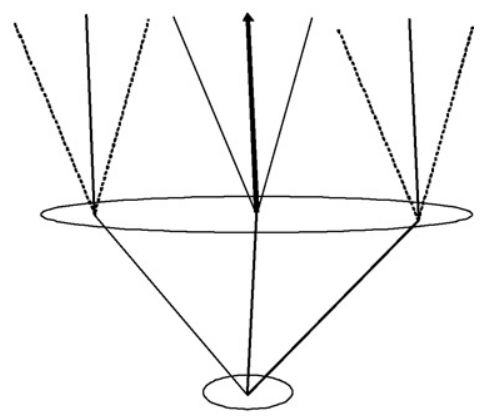

the active domain

Fig. 16. Nonconvergence example.

\begin{tabular}{llll} 
& \multicolumn{1}{c}{$\mathrm{s}_{21}$} & $\mathrm{~s}_{22}$ & $\mathrm{~s}_{23}$ \\
$\mathrm{~s}_{11}$ & $(3,3)^{N E}$ & $(2,2)$ & $(2,2)$ \\
$\mathrm{s}_{12}$ & $(2,2)$ & $(4,2)$ & $(2,4)$ \\
$\mathrm{s}_{13}$ & $(2,2)$ & $(2,4)$ & $(4,2)$
\end{tabular}

Fig. 17. Strategic form.

$$
\begin{array}{ll}
\mathrm{S}_{11} & 2 \\
\mathrm{~S}_{12} & 4 \\
\mathrm{~s}_{13} & 2
\end{array}
$$

Fig. 18. 1's view.

$$
\begin{array}{ccc}
\mathrm{s}_{21} & \mathrm{~s}_{22} & \mathrm{~s}_{23} \\
2 & 2 & 4
\end{array}
$$

Fig. 19. 2's view.

$$
\begin{array}{ll}
\mathrm{S}_{11} & 2 \\
\mathrm{~S}_{12} & 2 \\
\mathrm{~S}_{13} & 4
\end{array}
$$

Fig. 20. Next round for 1 .

By this revision, the regular behavior becomes $\left(\boldsymbol{s}_{12}, \boldsymbol{s}_{23}\right)$. After experiencing this pair as well as some trials, the personal views of the player's will be revised to the 1-person games summarized by the matrices of Figs. 20 and 21 .

With this new view, player 1 now finds that he should change his behavior, while player 2 does not. The revised behavior becomes $\left(\boldsymbol{s}_{13}, \boldsymbol{s}_{23}\right)$. In this manner, the players move cyclically through the four regular behaviors depicted in the bottom right corner of Fig. 22, and never converge to the Nash equilibrium $\left(\boldsymbol{s}_{11}, \boldsymbol{s}_{21}\right)$.

$$
\begin{array}{ccc}
\mathrm{S}_{21} & \mathrm{~S}_{22} & \mathrm{~S}_{23} \\
2 & 2 & 4
\end{array}
$$

Fig. 21. Next round for 2 .

$\begin{array}{rrrc} & \mathrm{s}_{21} & \mathrm{~s}_{22} & \mathrm{~s}_{23} \\ \mathrm{~s}_{11} & (3,3) & (2,2) & (2,2) \\ \mathrm{s}_{12} & (2,2) & (4,2) & \rightarrow(2,4) \downarrow \\ \mathrm{s}_{13} & (2,2) & \uparrow(2,4) & \leftarrow(4,2)\end{array}$

Fig. 22. Cycling. 


\section{8. g-Morphism analysis of decision making}

In Section 6, we showed, using the concept of a g-morphism, that the direct view can be regarded as a representative one. On the other hand, in Section 7, we assumed that a player makes a decision using the direct view $\left(\Gamma^{d}, \mathfrak{m}^{d}\right)$.Here, we apply the $g$-morphism analysis to the decision making of a player. The concept of a $g$-morphism helps us analyze decision making within some class of i.d.views. Here we do not restrict ourselves to the memory kits based on the SPR memory function $\mathfrak{m}_{i}^{\text {spr }}$ and on the active domain $D_{i}^{A}\left(\sigma^{*}\right)$. Although the $g$-morphism analysis works well, we still find some difficulties in decision making with personal views and in transitions from subjective optimality to objective behavior.

\subsection{Subjective optimality and g-morphism analysis}

Let $(\Gamma, \mathfrak{m})$ be a personal view of player $i$. We assume that $\Gamma$ satisfies $N=\{i\}$, i.e., it is a 1 -person game. We call such a view a purely personal view.

We compare subjective optimality across g-morphic views of player $i$. For this purpose, let $(\Gamma, \mathfrak{m})$ and $(\hat{\Gamma}, \hat{\mathfrak{m}})$ be two purely personal views of player $i$, and let $\sigma_{i} \in \Sigma_{i}$ and $\hat{\sigma}_{i} \in \hat{\Sigma}_{i}$. Here, we follow the convention that each notion in $(\hat{\Gamma}$, $\hat{\mathfrak{m}})$ is distinguished from the corresponding one in $(\Gamma, \mathfrak{m})$ by the "cap", e.g., $\Sigma_{i}$ and $\hat{\Sigma}_{i}$ are the sets of strategies of $(\Gamma, \mathfrak{m})$ and $(\hat{\Gamma}, \hat{\mathfrak{m}})$, respectively. We say that $\sigma_{i}$ and $\hat{\sigma}_{i}$ are endpiece-equivalent iff

$$
\lambda\left(\sigma_{i}\right)=\hat{\lambda}\left(\hat{\sigma}_{i}\right) .
$$

Recall that $\lambda\left(\sigma_{i}\right)$ is the set of compatible endpieces for $\sigma_{i}$, which was defined in (2.15). Endpiece-equivalent strategies $\sigma_{i}$ and $\hat{\sigma}_{i}$ lead to the same endpieces in $(\Gamma, \mathfrak{m})$ and $(\hat{\Gamma}, \hat{\mathfrak{m}})$. Using this notion, we show that it suffices to consider the direct view in order to study subjective optimality in a personal view.

The first theorem guarantees that the strategic consideration in either view of $(\Gamma, \mathfrak{m})$ and $(\hat{\Gamma}, \hat{\mathfrak{m}})$ can be carried over to the other as far as the compatible endpieces are concerned. The proofs will be given in the end of this subsection.

Theorem 8.1 (g-Morphism and behavior). Let $(\Gamma, \mathfrak{m})$ and $(\hat{\Gamma}, \hat{\mathfrak{m}})$ be two purely personal views of player $i$, and let $\psi$ be a g-morphism from $(\Gamma, \mathfrak{m})$ to $(\hat{\Gamma}, \hat{\mathfrak{m}})$.

(a) For each $\hat{\sigma}_{i} \in \hat{\Sigma}_{i}$, the function $\sigma_{i}$ defined by (8.2) is a strategy in $\Sigma_{i}$ and is endpiece-equivalent to $\hat{\sigma}_{i}$ : for all $x \in X_{i}^{D}$,

$$
\sigma_{i}(x)=\hat{\sigma}_{i} \cdot \psi(x) .
$$

(b) For each $\sigma_{i} \in \Sigma_{i}$, the function $\hat{\sigma}_{i}$ defined by (8.3) is a strategy in $\hat{\Sigma}_{i}$ and is endpiece-equivalent to $\sigma_{i}$ : for each $\hat{x} \in \hat{X}_{i}^{D}$,

$$
\hat{\sigma}_{i}(\hat{x})=\sigma_{i}(x) \text { for some } x \in X_{i}^{D} \text { with } \psi(x)=\hat{x} .
$$

Although Theorem 8.1 itself (as well as the following corollaries) does not refer to i.d.views, the theorem is more relevant in the context of i.d.views. Recalling the results of Section 6, Theorem 8.1 guarantees that our strategic analysis on a direct view is carried over to any other i.d.view that is g-morphic to that direct view.

We should consider the implications of Theorem 8.1 in two respects. One is in terms of subjective optimality, and the other is about when player $i$ brings back his modified behavior in the objective situation. From the viewpoint of $g$-morphisms, everything works well even in these respects. However, there are still some remaining difficulties in those two respects that are not captured by $g$-morphisms. These will be discussed in Section 8.2.

(1) g-Morphism and subjective optimality: Since we do not assume that $\mathfrak{m}_{i}^{\mathrm{o}}=\mathfrak{m}_{i}^{\mathrm{spr}}$ and $D_{i}^{\mathrm{o}}=D_{i}^{\mathrm{A}}\left(\sigma^{*}\right)$, some i.d.views may be extensive games only in the weak sense. In such cases, the utility maximization (7.3) in Section 7 needs some modification. Here, we give one possible modification.

Let $(\Gamma, \mathfrak{m})$ be a purely personal view of player $i$. A strategy $\sigma_{i}$ is subjectively optimal in $(\Gamma, \mathfrak{m})$ iff

$$
\min _{w \in \lambda\left(\sigma_{i}\right)} h(w) \geq \min _{w^{\prime} \in \lambda\left(\sigma_{i}^{\prime}\right)} h\left(w^{\prime}\right) \quad \text { for all } \sigma_{i}^{\prime} \in \Sigma_{i} .
$$

This is the maximin criterion for his decision making: the worst outcome compatible with this strategy is better than or equal to the worst outcome of any other strategy.

Corollary 8.1 (g-Morphism and subjective optimality). Let $(\Gamma, \mathfrak{m})$ and $(\hat{\Gamma}, \hat{\mathfrak{m}})$ be two purely personal views of player $i$, and let $\psi$ be a g-morphism from $(\Gamma, \mathfrak{m})$ to $(\hat{\Gamma}, \hat{\mathfrak{m}})$.

(a) If $\sigma_{i}$ satisfies (8.4) in $(\Gamma, \mathfrak{m})$, then the endpiece-equivalent strategy $\hat{\sigma}_{i}$ defined by (8.3) satisfies (8.4) in $(\hat{\Gamma}, \hat{\mathfrak{m}})$.

(b) If $\hat{\sigma}_{i}$ satisfies $(8.4)$ in $(\hat{\Gamma}, \hat{\mathfrak{m}})$, then the endpiece-equivalent strategy $\sigma_{i}$ defined by $(8.2)$ satisfies $(8.4)$ in $(\Gamma, \mathfrak{m})$.

Again, we talk about the corollary in the context of i.d.views. By the results of Section 6 , we can regard $(\hat{\Gamma}, \hat{\mathfrak{m}})$ as a direct one. By this result, we lose nothing in terms of subjective optimality by focusing on a direct view. 
(2) g-Morphism and objective behavior: After his decision making in an i.d.view, a player modifies his behavior pattern with his subjective strategy, and brings it back to the objective situation. This modification might depend upon the particular i.d.view of the player. In fact, we will show that the prescriptions for objective strategies are not different across $g$-morphic i.d.views. This implies that we can focus on the direct view even in the step of taking the prescription back to the objective world.

For the above consideration, we first modify (7.2) in the following way. Let $(\Gamma, \mathfrak{m})$ be a purely personal view of player $i$ and let $\sigma_{i}$ satisfy (8.4). We define the $p$ rescribed behavior of player $i$ in $\left(\Gamma^{\circ}, \mathfrak{m}^{\circ}\right)$ by: for all $x \in X_{i}^{\circ}$,

$$
\sigma_{i}^{1}(x)= \begin{cases}\sigma_{i}\left(x^{\prime}\right) & \text { if } \mathfrak{m}_{i}^{\circ}(x)=\mathfrak{m}\left(x^{\prime}\right) \text { for some } x^{\prime} \in X \\ \sigma_{i}^{\circ}(x) & \text { if } \mathfrak{m}_{i}^{0}(x) \neq \mathfrak{m}\left(x^{\prime}\right) \text { for any } x^{\prime} \in X\end{cases}
$$

This strategy prescribes the same behavior as (7.2) in the case of Section 7. The next corollary states that $g$-morphic views give the same prescriptions for behavior in the objective situation.

Corollary 8.2 (g-Morphism and modified behavior). Let $(\Gamma, \mathfrak{m})$ and $(\hat{\Gamma}, \hat{\mathfrak{m}})$ be two purely personal views of player $i$, and let $\psi$ be a g-morphism from $(\Gamma, \mathfrak{m})$ to $(\hat{\Gamma}, \hat{\mathfrak{m}})$.

(a) Let $\sigma_{i}$ be a strategy in $(\Gamma, \mathfrak{m})$, and let $\hat{\sigma}_{i}$ be the endpiece-equivalent strategy defined by $(8.3)$. Then $\sigma_{i}$ and $\hat{\sigma}_{i}$ prescribe the same behavior to player i in $\left(\Gamma^{\mathrm{o}}, \mathrm{m}^{\mathrm{o}}\right)$, that is, the modified behaviors defined by (8.5) with $\sigma_{i}$ and $\hat{\sigma}_{i}$ are the same.

(b) Let $\hat{\sigma}_{i}$ be a strategy in $(\hat{\Gamma}, \hat{\mathfrak{m}})$, and let $\sigma_{i}$ be the endpiece-equivalent strategy defined by (8.2). Then $\sigma_{i}$ and $\hat{\sigma}_{i}$ prescribe the same behavior to player i in $\left(\Gamma^{\mathrm{o}}, \mathrm{m}^{\mathrm{o}}\right)$.

In this corollary, we did not refer to the optimization condition (8.4). Of course, we can assume that $\sigma_{i}$ in (a) or $\hat{\sigma}_{i}$ in (b) satisfies (8.4). Although Corollary 8.1 states that subjective optimality is invariant with personal views, subjective optimality may not guarantee, in general, the objective optimality of the prescribed behavior in contrast to Theorem 7.1.

Now we prove Theorem 8.1 and the corollaries.

Proof of Theorem 8.1. (a) Let $\hat{\sigma}_{i} \in \hat{\Sigma}_{i}$. Consider the function $\sigma_{i}$ defined by (8.2). We show that $\sigma_{i}$ satisfies (1) and (2) for $(\Gamma, \mathfrak{m})$, and that it is endpiece-equivalent to $\hat{\sigma}_{i}$.

Since $\hat{\sigma}_{i} \cdot \psi(x) \in \hat{\mathrm{A}}_{\psi(x)}$ by (1) for $\hat{\sigma}_{i}$ and g4 requires $\hat{\mathrm{A}}_{\psi(x)}=A_{x}$, we have $\sigma_{i}(x) \in A_{x}$, i.e., $\sigma_{i}$ satisfies (1). To prove (2) for $\sigma_{i}$, consider $x, y \in X_{i}^{D}$ with $\mathfrak{m}(x)=\mathfrak{m}(y)$. Then, by $\mathrm{g} 7, \hat{\mathfrak{m}} \cdot \psi(x)=\hat{\mathfrak{m}} \cdot \psi(y)$. Since $\hat{\sigma}_{i}$ satisfies $(2)$, we have $\hat{\sigma}_{i} \cdot \psi(x)=\hat{\sigma}_{i} \cdot \psi(y)$, which means $\sigma_{i}(x)=\sigma_{i}(y)$.

Next we show that the two strategies are endpiece-equivalent. This has two parts, $\lambda\left(\sigma_{i}\right) \subseteq \hat{\lambda}\left(\hat{\sigma}_{i}\right)$ and $\hat{\lambda}\left(\hat{\sigma}_{i}\right) \subseteq \lambda\left(\sigma_{i}\right)$.

First, let $w \in \lambda\left(\sigma_{i}\right)$. Then, there is a play $\left\langle x_{1}, \ldots, x_{k}, x_{k+1}\right\rangle$ in $\Gamma$ with $\lambda\left(x_{t+1}\right)=w$ and $\theta\left(x_{k+1}\right)=$ $\left\langle\left(\lambda\left(x_{1}\right), \sigma_{i}\left(x_{1}\right)\right), \ldots,\left(\lambda\left(x_{k}\right), \sigma_{i}\left(x_{k}\right)\right), \lambda\left(x_{k+1}\right)\right\rangle$. We denote $\psi\left(x_{t}\right)$ by $\hat{x}_{t}$ for $t=1, \ldots, k+1$. By Lemma $6.4,\left\langle\hat{x}_{1}, \ldots, \hat{x}_{k+1}\right\rangle$ is a play in $\hat{\Gamma}$ and $\hat{\theta}\left(\hat{x}_{t+1}\right)=\theta\left(x_{k+1}\right)$. By g3, $\hat{\lambda}\left(\hat{x}_{t}\right)=\lambda\left(x_{t}\right)$ for $t=1, \ldots, k+1$, and by $(8.2), \hat{\sigma}_{i}\left(\hat{x}_{t}\right)=\sigma_{i}\left(x_{t}\right)$ for $t=1, \ldots, k$. Hence, $\hat{\theta}\left(\hat{x}_{t+1}\right)=\left\langle\left(\hat{\lambda}\left(\hat{x}_{1}\right), \hat{\sigma}_{i}\left(\hat{x}_{1}\right)\right), \ldots,\left(\hat{\lambda}\left(\hat{x}_{1}\right), \hat{\sigma}_{i}\left(\hat{x}_{k}\right)\right), \hat{\lambda}\left(\hat{x}_{k+1}\right)\right\rangle$, which means $w \in \hat{\lambda}\left(\hat{\sigma}_{i}\right)$.

Conversely, let $w \in \hat{\lambda}\left(\hat{\sigma}_{i}\right)$. Then, there is a play $\left\langle\hat{x}_{1}, \ldots, \hat{x}_{k}, \hat{x}_{k+1}\right\rangle$ in $\hat{\Gamma}$ with $\hat{\lambda}\left(\hat{x}_{t+1}\right)=w$ and $\hat{\theta}\left(\hat{x}_{t+1}\right)=$ $\left\langle\left(\hat{\lambda}\left(\hat{x}_{1}\right), \hat{\sigma}_{i}\left(\hat{x}_{1}\right)\right), \ldots,\left(\hat{\lambda}\left(\hat{x}_{1}\right), \hat{\sigma}_{i}\left(\hat{x}_{k}\right)\right), \hat{\lambda}\left(\hat{x}_{t+1}\right)\right\rangle$. By Lemma 6.4 , there is a play $\left\langle x_{1}, \ldots, x_{k}, x_{k+1}\right\rangle$ in $(\Gamma, \mathfrak{m})$ such that $\psi\left(x_{t}\right)=\hat{x}_{t}$ and $\hat{\theta}\left(\hat{x}_{t+1}\right)=\theta\left(x_{k+1}\right)$ for $t=1, \ldots, k+1$. By g3, $\hat{\lambda}\left(\hat{x}_{t}\right)=\lambda\left(x_{t}\right)$ for $t=1, \ldots, k+1$, and by $(8.2), \hat{\sigma}_{i}\left(\hat{x}_{t}\right)=\sigma_{i}\left(x_{t}\right)$ for $t=1, \ldots, k$. Hence, $\theta\left(x_{k+1}\right)=\left\langle\left(\lambda\left(x_{1}\right), \sigma_{i}\left(x_{1}\right)\right), \ldots,\left(\lambda\left(x_{k}\right), \sigma_{i}\left(x_{k}\right)\right), \lambda\left(x_{k+1}\right)\right\rangle$, which means $w \in \lambda\left(\sigma_{i}\right)$.

(b) Let $\sigma_{i} \in \Sigma_{i}$. Then, it holds that for all $x, y \in X^{D}$,

$$
\text { if } \psi(x)=\psi(y) \text { then } \sigma_{i}(x)=\sigma_{i}(y) .
$$

Suppose $\psi(x)=\psi(y)$. Then by $\mathrm{g} 7, \mathfrak{m}(x)=\mathfrak{m}(y)$. This and (2) imply $\sigma_{i}(x)=\sigma_{i}(y)$.

Next, we show that $\hat{\sigma}_{i}$ given in (8.3) is well-defined as a function over $\hat{X}_{i}^{D}$. Take an arbitrary $\hat{x} \in \hat{X}_{i}^{D}$. Since $\psi$ is a surjection, there is some $x \in X$ satisfying $\psi(x)=\hat{x}$. By Lemma $6.2, x \in X_{i}^{D}$. By (8.6), the particular $x$ chosen to satisfy $\psi(x)=\hat{x}$ does not affect the strategy $\hat{\sigma}_{i}(\hat{x})$. Hence, (8.3) defines a unique function $\hat{\sigma}_{i}$ for player $i$.

Once we show that $\hat{\sigma}_{i}$ satisfies (1) and (2), we will conclude that $\hat{\sigma}_{i}$ is a strategy in $(\hat{\Gamma}, \hat{\mathfrak{m}})$. By $(1)$ for $\sigma_{i}$ and g4, we have that $\hat{\sigma}_{i}(\hat{x}) \in \hat{\mathrm{A}}_{\hat{x}}=A_{x}$, i.e., $\hat{\sigma}_{i}$ satisfies (1). Next, let $\hat{x}, \hat{y} \in \hat{X}_{i}^{D}$ and $\hat{\mathfrak{m}}(\hat{x})=\hat{\mathfrak{m}}(\hat{y})$. Then, by g7, for any $x, y \in X_{i}^{D}$, if $\psi(x)=\hat{x}$ and $\psi(y)=\hat{y}$, then $\mathfrak{m}(x)=\mathfrak{m}(y)$. Since $\sigma_{i}$ satisfies (2), we have $\sigma_{i}(x)=\sigma_{i}(y)$, and $\hat{\sigma}_{i}(\hat{x})=\hat{\sigma}_{i}(\hat{y})$.

We can prove that $\hat{\sigma}_{i}$ and $\sigma_{i}$ are endpiece-equivalent in almost the same way as in the proof of (a) using (8.3) in place of (8.2).

Proof of Corollary 8.1(a). Let $\sigma_{i}$ satisfy $(8.4)$ in $(\Gamma, \mathfrak{m})$, and let $\hat{\sigma}_{i}$ be the endpiece-equivalent strategy defined by (8.3). By g3, g6, and endpiece-equivalence of $\sigma_{i}$ and $\hat{\sigma}_{i}$, we have $\min _{\hat{w} \in \hat{\lambda}\left(\hat{\sigma}_{i}\right)} \hat{h}(\hat{w})=\min _{w \in \lambda\left(\sigma_{i}\right)} h(w)$. For each $\hat{\sigma}_{i}^{\prime} \in \hat{\Sigma}_{i}$, Theorem 8.1 guarantees that there is an endpiece-equivalent strategy $\sigma_{i}^{\prime} \in \Sigma_{i}$ defined by (8.2) and $\min _{\hat{w}^{\prime} \in \hat{\lambda}\left(\hat{\sigma}_{i}^{\prime}\right)} \hat{h}\left(\hat{w}^{\prime}\right)=\min _{w^{\prime} \in \lambda\left(\sigma_{i}^{\prime}\right)} h\left(w^{\prime}\right)$. Hence, since $\sigma_{i}$ satisfies (8.4) in $(\Gamma, \mathfrak{m})$, we have, $\min _{\hat{w} \in \hat{\lambda}\left(\hat{\sigma}_{i}\right)} \hat{h}(\hat{w}) \geq \min _{\hat{w}^{\prime} \in \lambda\left(\hat{\sigma}_{i}^{\prime}\right)} h\left(\hat{w}^{\prime}\right)$ for all $\hat{\sigma}_{i}^{\prime} \in \hat{\Sigma}_{i}$.

Part (b) is proved in almost the same way.

Proof of Corollary 8.2(a). Let $\sigma_{i}$ satisfy $(8.4)$ in $(\Gamma, \mathfrak{m})$, and let $\hat{\sigma}_{i}$ be the strategy defined by (8.3). By Corollary 8.2, $\hat{\sigma}_{i}$ satisfies (8.4) in $(\hat{\Gamma}, \hat{\mathfrak{m}})$. We let $\sigma_{i}^{1}(x)$ and $\hat{\sigma}_{i}^{1}(x)$ denote the behavior prescribed by $(8.5)$ in $(\Gamma, \mathfrak{m})$ and $(\hat{\Gamma}, \hat{\mathfrak{m}})$, respectively. Let $x \in X_{i}^{\circ}$. 


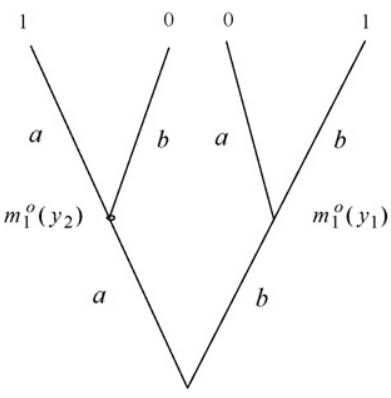

(A)

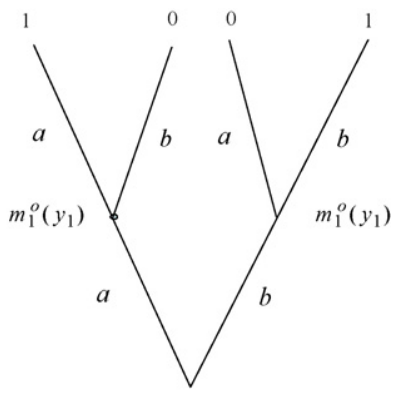

(B)

Fig. 23. Difficulty in objective optimality.

If $\mathfrak{m}_{i}^{\circ}(x)=\mathfrak{m}\left(x^{\prime}\right)$ for some $x^{\prime} \in X$, then by g0 there is an $\hat{x}^{\prime} \in \hat{X}$ where $\hat{x}^{\prime}=\psi\left(x^{\prime}\right)$. By $(8.3), \hat{\sigma}_{i}\left(\hat{x}^{\prime}\right)=\sigma_{i}\left(x^{\prime}\right)$, so $\sigma_{i}^{1}(x)=\hat{\sigma}_{i}^{1}(x)$. If, alternatively, $\mathfrak{m}_{i}^{\circ}(x) \neq \mathfrak{m}\left(x^{\prime}\right)$ for any $x^{\prime} \in X$, then $\sigma_{i}^{1}(x)=\sigma_{i}^{0}(x)=\hat{\sigma}_{i}^{1}(x)$. Part (b) is proved in almost the same way as (a).

\subsection{Difficulties involved in subjective thinking and in playing in the objective situation}

In Section 7, we assumed that player $i$ has the memory function $\mathfrak{m}_{i}^{\mathrm{o}}=\mathfrak{m}_{i}^{\mathrm{spr}}$ and the active domain $D_{i}^{\mathrm{A}}\left(\sigma^{\mathrm{o}}\right)$. Then, he succeeds in having the unique direct view, in finding an optimal strategy in $\left(\Gamma^{d}, \mathrm{~m}^{d}\right)$ as well as in bringing it back to the objective situation. However, if we drop these assumptions, then a subjectively optimal strategy may not help him behave properly in the objective situation. We can find many difficulties in decision making here, but we restrict ourselves to only some of them.

(1) Difficulty in subjective thinking: We start with a difficulty involved in subjective thinking. In Corollary 5.1, we gave a necessary and sufficient condition for a direct view to be unique and inductively derived. When the direct view is uniquely determined, the results of Section 6 state that it is essentially the smallest i.d.view. Also, the results of Section 8.1 imply that decision making is invariant to the choice of a personal view.

Problems may arise because of multiplicity of direct views for a given memory kit $\left(T_{D_{i}}, \mathcal{Y}_{D_{i}}\right)$. In this case, player $i$ faces a difficulty first in choosing an i.d.view.

In Example 5.1 there are four direct i.d.views, which all differ in terms of the memory function. Fig. 23 gives two of those direct i.d.views with only the relevant memory yarns listed, and the payoffs are now attached. In Fig. 23 A, the memory yarns are mixed up at the nodes $\left\langle\left(y_{0}, a\right), v\right\rangle$ and $\left\langle\left(y_{0}, b\right), v\right\rangle$ as $\mathfrak{m}_{1}^{\circ}\left(y_{2}\right)$ and $\mathfrak{m}_{1}^{\circ}\left(y_{1}\right)$, while the objective game has the same structure with the opposite assignment of $\mathfrak{m}_{1}^{o}\left(y_{2}\right)$ and $\mathfrak{m}_{1}^{o}\left(y_{1}\right)$. In Fig. $23 \mathrm{~B}$, he expects the same memory yarn $\mathfrak{m}_{1}^{\circ}\left(y_{1}\right)$ at each of his second decision nodes. In the view $A$, he does not use the memory yarn $\mathrm{m}_{1}^{\circ}\left(y_{2}\right)$ in $\mathcal{Y}_{D_{1}}$. This multiplicity of views causes some difficulty for the player in deciding which view to use for his decision making. His choice of a view may influence his decision making since, e.g., in the view A he can make different choices at $\left\langle\left(y_{0}, a\right), v\right\rangle$ and $\left\langle\left(y_{0}, b\right), v\right\rangle$, while in view $\mathrm{B}$, he is required to make the same choice.

(2) Difficulty in objective optimality: Suppose that player 1 has chosen an direct i.d.view and a behavior pattern for it that is subjectively optimal in the sense of (8.4). Consider the direct view of Fig. 23 A. One subjectively optimal strategy is defined by $\sigma_{1}$ choosing action $a$ at the root node and the left node with $\mathfrak{m}_{1}^{\circ}\left(y_{2}\right)$, while choosing $b$ at the right node with $\mathfrak{m}_{1}^{\circ}\left(y_{1}\right)$. When he modifies his regular behavior in the objective game by this strategy $\sigma_{1}$ and brings it back to the objective situation, he receives the payoff 0 . Thus he fails to behave optimally in the objective situation.

Next, consider the view B. In this view, he has a subjectively optimal strategy prescribing the choice of $b$ at all the decision nodes. If he takes this strategy to the objective world, he will receive the memory yarn $\mathfrak{m}_{1}^{o}\left(y_{2}\right)$, which he does not expect and, indeed, is not contained in his constructed personal view. Thus, the player finds a further difficulty with his view and a reason to revise his behavior or his view.

This difficulty is caused by the weak inclusion condition of P2a, allowing the possibility of $\left\{\mathfrak{m}^{i}(x): x \in X_{i}^{i}\right\} \subsetneq \mathcal{Y}_{D_{i}}$. By strengthening P2a to equality, this difficulty could be avoided as in the view B. Nevertheless, the multiplicity of views remains, and so does the difficulty that a subjectively optimal strategy may not be objectively optimal.

Thus, when there are multiple direct i.d.views, player $i$ may meet some difficulties both subjectively and objectively. Either of these difficulties gives a player a reason to revise his behavior or his view. In this paper, however, we do not consider those revisions.

\section{Concluding comments}

We have given a discourse of inductive game theory by confining ourselves to clear-cut cases. It would be, perhaps, appropriate to start this section with comments on our discourse. Then we will discuss some implications for extant game theory. 


\subsection{Comments on our discourse}

We have made particular choices of assumptions and definitions for our discourse. One important methodological choice is to adopt extensive games in the strong and weak senses for objective and subjective descriptions. First, we will give some comments on this choice, and then, we will discuss the definition of an inductively derived view given in Section 4 based on the initial segment procedure.

As pointed out in Section 4, an extensive game contains observable and unobservable elements. The nodes with the successor relation are unobservable for the players and even for the outside observer, in which sense those are highly hypothetical. The components in a memory kit are all observables and actually observed. Thus, our definition of the inductive derivation of a personal view from a memory kit extends the observed observables by adding hypothetical elements. This may be interpreted as an "inductive" process of adding unobservable elements to observed data. However, this freedom of adding hypothetical elements leads us a proliferation of possible views. To prevent this proliferation, we need some criterion to choose a view from many possible ones. In this paper, we have used the concept of a g-morphism (game theoretical p-morphism) to choose a smallest one.

Conceptually speaking, the choice of a personal view is supposed to be done by a player, rather than us. While the definition of an inductive derivation allows many views, a player cannot construct a large one because of his bounded cognitive ability. Thus, the criteria of smallness and constructiveness are important from this point of view. The direct view defined in Section 5 has a constructive nature as well as being a smallest one for a given memory kit. In this sense, the direct view has a special status among those possible views.

Nevertheless, Definition 4.1 may admit no inductively derived views for a given memory kit, as characterized by Theorem 5.1. In fact, the initial segment procedure adopted in Definition 4.1 still gives a strong restriction on the addition of hypothetical elements. If we allow more freedom in using hypothetical elements in an inductive derivation, we could avoid the nonexistence result. For example, if we allow a player to add "nature nodes" to his personal view, we could even avoid the use of an extensive game in the weak sense. On the other hand, this creates vast arbitrariness in inductive derivations; and we expect serious difficulties in finding natural criteria to narrow down the use of "nature nodes". Until we find natural criteria, we should refrain from the cheap use of "nature nodes".

The above conclusion may sound negative to any extension of our definition of an inductive derivation, but we have different opinions. We could actually have a more general procedure to construct a personal view than the initial segment procedure. Since this paper is intended to provide an entire scenario, we have chosen the initial segment procedure as a clear-cut case. In separate papers, we will discuss less restrictive definitions. See Section 9.3.

Another comment should be given on the choice of extensive games. In fact, we can avoid the adoption of extensive games; instead, the present authors (Kaneko and Kline, in press-a) have developed a theory of information protocols, which avoids the use of nodes and describes game situations directly in terms of information pieces and actions together with a history-event relation. If we adopt this theory, then we could avoid a proliferation of personal views generated by the use of hypothetical nodes. In the theory of information protocols it may be easier to discuss extensions of inductive derivations. One reason for our adoption of extensive games here is their familiarity within our profession. The choice of extensive games makes the distinction between observables and unobservables explicit, which is another reason for our choice.

We expect gradual developments of inductive game theory to come about by deeper analysis and alternative approaches to the various stages mentioned in the diagram of Fig. 1. By such gradual developments, we may find natural criteria for steps such as the use of nature nodes, and some experimental tests of inductive game theory.

\subsection{Some implications to extant game theory}

It is a main implication of our discourse that a good individual view on society is difficult to construct from the experiential point of view: there are many places for a player to get stuck in his inductive process and analysis process. Nevertheless, we gave a characterization theorem of Nash equilibrium in Section 7. Here, we discuss some other implications to extant game theory and economics chiefly with respect to Nash equilibrium.

There are various interpretations of Nash equilibrium (cf. Kaneko, 2004, Act 4). Nash (1951) himself described his concept from the viewpoint of purely ex ante decision making, but in economic applications, it is typically more natural to interpret Nash equilibrium as a strategically stable stationary state in a recurrent situation. The characterization given in Section 7 is along this line of interpretations, including also ex ante decision making in a player's constructed personal view.

To reach Nash equilibrium, which may not be the case, it takes a long time. Also, the process of trial and error may not allow all possible available actions. The Nash equilibrium reached should be regarded as a Nash equilibrium in the game with respect to the actually experienced domains. Thus, the characterization of Nash equilibrium in Section 7 should not merely be interpreted as a positive result. It means that the characterization would be obtained if all those processes go through well and if reservations about restrictions on trials are taken into account.

From the same point of view, the subgame perfect equilibrium of Selten (1975) involves even deeper difficulties from our experiential point of view, which was already pointed out in Kaneko and Matsui (1999). The reason is that subgame perfection requires higher order experimentations. When one player deviates from his regular behavior, other players in turn need, again, to make experimentations from regular behavior. This second or higher order experimentation is already problematic and violates some principles discussed in the informal theory in Section 3.2. In fact, a similar criticism is applied 
to Nash equilibrium, as already stated. Nash equilibrium itself is regarded as one limit notion, and subgame perfection is a higher limit one.

Taking the above criticism seriously, one important problem arises. The complexities, in a certain sense, of an inductively derived view as well as of experimentations are measured and restricted. In the epistemic logic context, Kaneko and Suzuki (2005) introduced the concept contentwise complexity, which measures "contentwise complexity" of a single instance of a game. This notion can be converted to our inductive game theory. Then, we will be able to give restrictions on individual views as well as experiments. In this manner, our inductive game theory will be developed in the direction of "bounded rationalities".

We have restricted our attention to the purely experiential sources. In our society, usually, we have different sources of beliefs/knowledge such as from other people or through education. These suggest that a player may get more beliefs/knowledge on the social structure, but do not suggest that he can guess other people's thinking, which has usually been assumed in the standard game theory (cf., Harsanyi, 1967 for incomplete information game and Kaneko, 2002 for the epistemic logic approach). At least, the assumption of common knowledge is far beyond experiences. If we restrict interpersonal thinking to very shallow levels, deductive game theory may have some connections to inductive game theory (cf. Kaneko and Suzuki, 2002 for such a direction of deductive game theory).

\subsection{Postscript}

By now, several new developments along the line of the scenario given in this paper have been made in Kaneko and Kline (2007a, 2007b, 2008) and Akiyama et al. (in press). We use this postscript section to present some small summaries of those papers to help the reader catch up to the present state of inductive game theory.

The main concern of Kaneko and Kline (2007) is the size of an inductively derived view for a player with bounded cognitive abilities. If the objective situation is too large, a player may have difficulty: (1) analyzing it strategically; and (2) accumulating enough experiences to have a rich view. The premise of that paper is that the number of experiences and the size of a view must be small for it to be managed by a player. The concept of "marking" some parts and actions as important was introduced in that paper and shown to be successful in allowing a player to obtain a manageable, though potentially biased, view.

As already mentioned in Section 9.1, Kaneko and Kline (2007b) introduced a new construct called an "information protocol", based on "actions" and "information pieces" as tangible elements for each player rather than hypothetical non-tangible concepts such as nodes. This approach gives a more direct and simpler description of a game situation from the perspective of a player. It has another merit to classify extensive games in a more clear-cut manner. With an appropriate choice of axioms, it fully characterizes an extensive game in the weak and strong senses. It also enables us to avoid g-morphisms, since we have no multiplicity in i.d.views caused by hypothetical nodes and branches. The theory of information protocols has been adopted in our more recent research including Kaneko and Kline (2008).

Kaneko and Kline (2008) took up that task of constructing i.d.views with more partiality in a players memory. Accordingly, the definition of an i.d.view had to be weakened to admit a view. By these generalizations, the induction becomes less deterministic and we meet some multiplicity of consistent views with a given set of memories. The interactions between a player's i.d.view, his future behavior, and future views become the topics of this paper and also serve as potential sources for resolving the multiplicity problem.

Finally, Akiyama et al. (in press) took a computer simulation approach in order to look into the process of experiencing and memorizing experiences in a one-person problem called "Mike's bike commuting". That paper tries to clarify the informal theory of behavior and accumulation of memories discussed in Section 3.2 of this paper. The simulation approach is based on finite experiences and accumulations of memories. The use of "marking" introduced in Kaneko and Kline (2007a) was found to be crucial for obtaining a rich enough view.

These developments are, more or less, consistent with the scenario spelled out in this paper and give more details into each step in the basic scenario. We are presently continuing our research along those lines making progress into experiential foundations of beliefs/knowledge on other players' thinking.

\section{Acknowledgements}

We thank Chih Chang, Takashi Ikegami and Ryuichiro Ishikawa for comments on earlier drafts of this paper. Also, we are grateful for hospitality of the institute of economics at Academia Sinica, Taiwan: some part of this paper was done during the visit of the authors to the institute.

The authors are partially supported by Grant-in-Aids for Scientific Research No. 18330034, Ministry of Education, Science and Culture, and Australian Research Council Discovery Grant DP0560034.

\section{References}

Akiyama, E., Ishikawa, R., Kaneko, M., Kline, J.J., in press. A simulation study of learning a structure: Mike's bike commuting. to appear in Economic Theory. University of Tsukuba. http://www.sk.tsukuba.ac.jp/SSM/libraries/pdf1176/1190.pdf.

Bacon, F., 1889, 1589. Novum Organum, edited with Introduction, Notes, etc., by Thomas Fowler, 2nd ed. Oxford.

Blackburn, P., De Rijke, M., Venema, Y., 2002. Modal Logic. Cambridge University Press, Cambridge.

Brandenburger, A., Dekel, E., 1993. Hierarchies of beliefs and common knowledge. Journal of Economic Theory 59, 189-198. 
Camerer, C., 2003. Behavioral Game Theory. Princeton University Press, Princeton.

Dubey, P., Kaneko, M., 1984. Information patterns and Nash equilibria in extensive games I. Mathematical Social Sciences 8, $111-139$.

Fudenberg, D., Levine, D.K., 1993. Self-confirming equilibrium. Econometrica 61, 523-545.

Gilboa, I., Schmeidler, D., 1995. Case-based decision theory. Quarterly Journal of Economics 110, 605-639.

Harper, W.L., Schulte, O., 2005. Scientific Method. McMillan Encyclopaedia of Philosophy.

Harsanyi, J.C., 1967/1968. Games with Incomplete Information Played by ‘Bayesian’ Players, Parts I,II, and III. Management Sciences 14, pp. 159-182, 320-334, $486-502$.

Hume, D., 1889, 1759. An Enquiry Concerning Human Understanding. Logmans, Green and Co., London.

Kalai, E., Lehrer, E., 1993. Subjective equilibrium in repeated games. Econometrica 61, 1231-1240.

Kaneko, M., 2002. Epistemic logics and their game theoretical applications: introduction. Economic Theory $19,7-62$.

Kaneko, M., 2004. Game Theory and Mutual Misunderstanding. Springer, Heidelberg.

Kaneko, M., Kline, J.J., 2007a. Small and Partial Views derived from Limited Experiences, SSM.DP.1166, University of Tsukuba. http://www.sk.tsukuba.ac. jp/SSM/libraries/pdf1151/1166.pdf.

Kaneko, M., Kline, J.J., 2007b. Information Protocols and Extensive Games in Inductive Game Theory. International Journal of Mathematics, Game Theory and Algebra 17 (5/6). Nova Publishers, New York.

Kaneko, M., Kline, J.J., 2008. Reciprocal effects between memories, views, and behavior. Economic Theory.

Kaneko, M., Matsui, A., 1999. Inductive game theory: discrimination and prejudices. Journal of Public Economic Theory 1, 101-137. Errata: the same journal 3 (2001), p. 347.

Kaneko, M., Suzuki, N.-Y., 2002. Bounded interpersonal inferences and decision making. Economic Theory 19, 63-103.

Kaneko, M., Suzuki, N.-Y., 2005. Contentwise Complexity of Inferences in Epistemic Logics of Shallow Depths I: General Development. University of Tsukuba, Mimeo.

Kuhn, H.W., 1953. Extensive Games and the Problem of Information, Contributions to the Theory of Games II. In: Kuhn, H.W., Tucker, A.W. (Eds.), Princeton University Press, pp. 193-216.

Kuhn, T., 1964. The Structure of Scientific Revolutions. Chicago University Press, Chicago.

Luce, R.D., Raiffa, H., 1957. Games and Decisions. John Wiley and Sons Inc., Boston.

Mertens, J., Zamir, S., 1985. Formulation of Bayesian analysis for games with incomplete information. International Journal of Game Theory $14,1-29$.

Nash, J.F., 1951. Noncooperative games. Annals of Mathematics 54, 286-295.

Ono, H., 1994. Logic in Information Sciences (in Japanese). Nihon-hyoron-sha, Tokyo.

Osborne, M., Rubinstein, A., 1994. A Course in Game Theory. MIT Press, Cambridge.

Piccione, M., Rubinstein, A., 1997. On the interpretation of decision problems with imperfect recall. Games and Economic Behavior $20,3-24$.

Ritzberger, K., 2002. Foundations of Non-cooperative Game Theory. Oxford University press, Oxford.

Selten, R., 1975. Reexamination of the perfectness concept of equilibrium points in extensive games. International Journal of Game Theory 4, 25-55.

von Neumann, J., Morgenstern, O., 1944. Theory of Games and Economic Behavior. Princeton University Press, Princeton.

Weibull, J.W., 1995. Evolutionary Game Theory. MIT Press, London. 


\title{
Erratum: Inductive Game Theory: A Basic Scenario ${ }^{1,2}$
}

\author{
by Mamoru Kaneko ${ }^{3}$ and J. Jude Kline ${ }^{4}$ \\ Journal of Mathematical Economics 44 (2008) 1332-1363
}

This erratum corrects a mistake in Theorem 8.1.(a). The following example given in Fig.1 shows that Theorem 8.1.(a) does not hold under the conditions given in the paper. Fig.1 is a simplification of Fig.13 in the paper. In both games $\Gamma$ and $\hat{\Gamma}$, the information functions are the identity functions. In addition, the memory function is assumed to be the perfect-information memory function $\mathfrak{m}^{P I}$ in $\Gamma\left(\hat{\mathfrak{m}}^{P I}\right.$ in $\left.\hat{\Gamma}\right)$. Now, let $\psi$ the function from $X$ to $\hat{X}$ defined by $\psi(y)=\hat{y}$ for all $y \neq x_{2}$ but $\psi\left(x_{2}\right)=\hat{x}_{1}$. Then, this $\psi$ is a $g$-morphism from $\Gamma$ to $\hat{\Gamma}$ in the sense of Definition 6.1 .

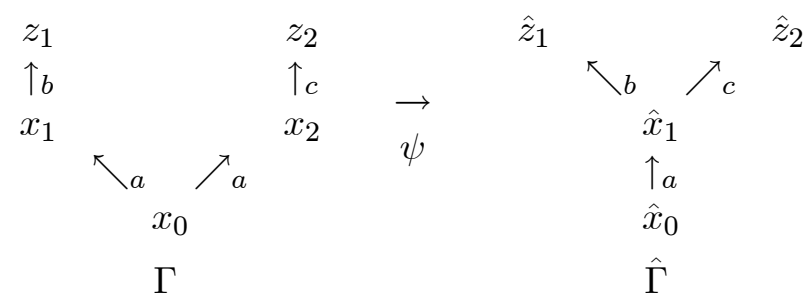

Fig.1

In the above example, Theorem 8.1.(a) fails: It states that for every strategy $\hat{\sigma}_{i}$ in $\hat{\Gamma}$, the function defined by $\sigma_{i}(y)=\hat{\sigma}_{i} \cdot \psi(y)$ for all nodes $y$ in $\Gamma$ is a strategy in $\Gamma$ and these strategies lead to the corresponding (same) endpieces. In the above example, if $\hat{\sigma}_{i}\left(\hat{x}_{1}\right)=c$, then $\sigma_{i}\left(x_{1}\right)=c$, which violates $(2.12)$ of the paper. Hence, this $\sigma_{i}$ is not a strategy in $\Gamma$; (a) does not hold.

One possible amendment to recover Theorem 8.1.(a) is to strengthen the concept of a $g$-morphism. We add one condition to Definition 6.1:

$\mathrm{g} 2^{*}$ : for all $\hat{x}, \hat{y} \in \hat{X}, x \in X$, and $a \in \hat{A}_{\hat{x}}, \hat{x} \hat{<}_{a} \hat{y}$ and $\hat{x}=\psi(x)$ imply $x<_{a} y$ and $\hat{y}=\psi(y)$ for some $y \in X$.

If a $g$-morphism satisfies this condition, then we call $\psi$ a strong $g$-morphism from $(\Gamma, \mathfrak{m})$ to $(\hat{\Gamma}, \hat{\mathfrak{m}})$.

\footnotetext{
${ }^{1}$ Correponding Author: Mamoru kaneko, kaneko@sk.tsukuba.ac.jp

${ }^{2}$ We thank Paddy Krishnan, Shravan Luckraz, and Phil Stocks for discussions leading us to find our mistake.

${ }^{3}$ Institute of Policy and Planning Sciences, University of Tsukuba, Ibaraki 305-8573, Japan (kaneko@shako.sk.tsukuba.ac.jp)

${ }^{4}$ Department of Economics, School of Business, Bond University, Gold Coast, QLD 4229, Australia, (Jeffrey_Kline@bond.edu.au)
} 
Condition g2* is independent of the other conditions g0-g7. In $\hat{\Gamma}$ of Fig. $1, \hat{x}_{1} \hat{<}_{b} \hat{z}_{1}$, and $\psi\left(x_{2}\right)=\hat{x}_{1}$. But we do not have $x_{2}<_{b} y$ for any $y \in X$. Hence, this $\psi$ does not satisfy condition g2* i.e., $\psi$ is a $g$-morphism but not a strong $g$-morphism.

Now, we re-state Theorem 8.1.(a) using a strong $g$-morphism, but note that (b) holds for a $g$-morphism. For completeness, we prove this theorem. We will find that condition $\mathrm{g}^{*}$ is used only to guarantee that the derived function $\sigma_{i}$ satisfies $(2.12)$; the above example captures entirely the falsity of the original version of Theorem 8.1.(a).

Theorem 8.1 (Strong $g$-Morphism and Behavior). Let $(\Gamma, \mathfrak{m})$ and $(\hat{\Gamma}, \hat{\mathfrak{m}})$ be two purely personal views of player $i$.

(a): Let $\psi$ be a strong $g$-morphism from $(\Gamma, \mathfrak{m})$ to $(\hat{\Gamma}, \hat{\mathfrak{m}})$. For each $\hat{\sigma}_{i} \in \hat{\Sigma}_{i}$, the function $\sigma_{i}$ defined by $(0.1)$ is a strategy in $\Sigma_{i}$ and is endpiece-equivalent to $\hat{\sigma}_{i}$ : for all $x \in X_{i}^{D}$,

$$
\sigma_{i}(x)=\hat{\sigma}_{i} \cdot \psi(x) .
$$

(b): Let $\psi$ be a $g$-morphism from $(\Gamma, \mathfrak{m})$ to $(\hat{\Gamma}, \hat{\mathfrak{m}})$. For each $\sigma_{i} \in \Sigma_{i}$, the function $\hat{\sigma}_{i}$ defined by $(0.2)$ is a strategy in $\hat{\Sigma}_{i}$ and is endpiece-equivalent to $\sigma_{i}$ : for each $\hat{x} \in \hat{X}_{i}^{D}$,

$$
\hat{\sigma}_{i}(\hat{x})=\sigma_{i}(x) \text { for some } x \in X_{i}^{D} \text { with } \psi(x)=\hat{x} .
$$

Proof.(a): Let $\hat{\sigma}_{i} \in \hat{\Sigma}_{i}$. Consider the function $\sigma_{i}$ defined by (0.1). We show that $\sigma_{i}$ satisfies $(2.12)$ and $(2.13)$ on $(\Gamma, \mathfrak{m})$, and that it is endpiece-equivalent to $\hat{\sigma}_{i}$.

Consider $x \in X_{i}^{D}$. Let $\hat{x}=\psi(x)$ and $\hat{\sigma}_{i}(\hat{x})=a$. By Lemma 6.4, we have $\hat{x} \in \hat{X}_{i}^{D}$. Then, by (2.12) for $\hat{\sigma}_{i}$, we have $\hat{\varphi}_{\hat{x}}(\hat{y})=a$ for some $\hat{y} \in \hat{X}_{i}$, and hence, $\hat{x} \hat{<}_{a} \hat{y}$. By g2*, $x<_{a} y$ for some $y \in X$. Then, this $y$ can be taken to be an immediate successor of $x$. Hence, $\sigma_{i}$ satisfies $(2.12)$ in $(\Gamma, m)$.

To prove (2.13) for $\sigma_{i}$ defined by $(0.1)$, consider $x, y \in X_{i}^{D}$ with $\mathfrak{m}(x)=\mathfrak{m}(y)$. Then, by g7, $\hat{\mathfrak{m}} \cdot \psi(x)=\mathfrak{m}(x)=\mathfrak{m}(y)=\hat{\mathfrak{m}} \cdot \psi(y)$. Since $\hat{\sigma}_{i}$ satisfies (2.13), we have $\hat{\sigma}_{i} \cdot \psi(x)=\hat{\sigma}_{i} \cdot \psi(y)$, which means $\sigma_{i}(x)=\sigma_{i}(y)$.

Next we show that the two strategies are endpiece-equivalent. This has two parts, $\lambda\left(\sigma_{i}\right) \subseteq \hat{\lambda}\left(\hat{\sigma}_{i}\right)$ and $\hat{\lambda}\left(\hat{\sigma}_{i}\right) \subseteq \lambda\left(\sigma_{i}\right)$. We show the former. The latter is proved in the same way.

First, let $w \in \lambda\left(\sigma_{i}\right)$. Then, there is a play $\left\langle x_{1}, \ldots, x_{k}, x_{k+1}\right\rangle$ in $\Gamma$ with $\lambda\left(x_{k+1}\right)=w$ and $\theta\left(x_{k+1}\right)=\left\langle\left(\lambda\left(x_{1}\right), \sigma_{i}\left(x_{1}\right)\right), \ldots,\left(\lambda\left(x_{k}\right), \sigma_{i}\left(x_{k}\right)\right), \lambda\left(x_{k+1}\right)\right\rangle$. We denote $\psi\left(x_{t}\right)$ by $\hat{x}_{t}$ for $t=1, \ldots, k+1$. By Lemma $6.4,\left\langle\hat{x}_{1}, \ldots, \hat{x}_{k+1}\right\rangle$ is a play in $\hat{\Gamma}$ and $\hat{\theta}\left(\hat{x}_{t+1}\right)=\theta\left(x_{k+1}\right)$. By g3, $\hat{\lambda}\left(\hat{x}_{t}\right)=\lambda\left(x_{t}\right)$ for $t=1, \ldots, k+1$, and by $(0.1), \hat{\sigma}_{i}\left(\hat{x}_{t}\right)=\sigma_{i}\left(x_{t}\right)$ for $t=1, \ldots, k$. Hence, $\hat{\theta}\left(\hat{x}_{t+1}\right)=\left\langle\left(\hat{\lambda}\left(\hat{x}_{1}\right), \hat{\sigma}_{i}\left(\hat{x}_{1}\right)\right), \ldots,\left(\hat{\lambda}\left(\hat{x}_{1}\right), \hat{\sigma}_{i}\left(\hat{x}_{k}\right)\right), \hat{\lambda}\left(\hat{x}_{k+1}\right)\right\rangle$, which means $w \in \hat{\lambda}\left(\hat{\sigma}_{i}\right)$.

(b): Let $\sigma_{i} \in \Sigma_{i}$. Then, it holds that for all $x, y \in X_{i}^{D}$,

$$
\text { if } \psi(x)=\psi(y) \text {, then } \sigma_{i}(x)=\sigma_{i}(y) .
$$

Indeed, if $\psi(x)=\psi(y)$, then by $\mathrm{g} 7, \mathfrak{m}(x)=\mathfrak{m}(y)$, which together with (2.13) for $\sigma_{i}$ implies $\sigma_{i}(x)=\sigma_{i}(y)$. Since $\psi$ is a surjection, for any $\hat{x} \in \hat{X}_{i}^{D}, \psi(x)=\hat{x}$ for some 
$x \in X$. By Lemma 6.4, we have $x \in X_{i}^{D}$. It follows from these facts that $\hat{\sigma}_{i}$ given in $(0.2)$ is well-defined as a function over $\hat{X}_{i}^{D}$. It remains to show (2.12) and (2.13) for $\hat{\sigma}_{i}$.

Consider (2.12) for $\hat{\sigma}_{i}$. Let $\hat{x} \in \hat{X}_{i}^{D}$. By g0, we have an $x \in X$ with $\psi(x)=\hat{x}$. By Lemma $6.4, x \in X_{i}^{D}$. Then, by (2.12) for $\sigma_{i}$, we have a $y \in X$ so that $\varphi_{x}(y)=\sigma_{i}(x)$. Let $\sigma_{i}(x)=a$. Then, $x<_{a} y$. By g1, we have $\psi(x) \hat{<}_{a} \psi(y)$, and by Lemma $6.4, \psi(y)$ is an immediate successor of $\psi(x)$. Thus, $\varphi_{\hat{x}}(\psi(y))=a$, which implies (2.12) for $\hat{\sigma}_{i}$.

Consider (2.13) for $\hat{\sigma}_{i}$. Let $\hat{x}, \hat{y} \in \hat{X}_{i}^{D}$ and $\hat{\mathfrak{m}}(\hat{x})=\hat{\mathfrak{m}}(\hat{y})$. By g0 (surjection), for any $x, y \in X_{i}^{D}$, we can find $x$ and $y$ so that $\psi(x)=\hat{x}$ and $\psi(y)=\hat{y}$. By $\mathrm{g} 7, \mathfrak{m}(x)=$ $\hat{\mathfrak{m}} \cdot \psi(x)=\hat{\mathfrak{m}} \cdot \psi(y)=\mathfrak{m}(y)$. Hence, by (2.13) for $\sigma_{i}$, we have $\sigma_{i}(x)=\sigma_{i}(y)$. By $(0.2)$, we have $\hat{\sigma}_{i}(\hat{x})=\hat{\sigma}_{i}(\hat{y})$.

We can prove that $\hat{\sigma}_{i}$ and $\sigma_{i}$ are endpiece-equivalent in almost the same way as in the proof of (a) using (0.2) in place of (0.1).

In the parallel manner, Corollaries 8.1 and 8.2 also should be corrected, replacing a g-morphism by a strong g-morphism in the parts, (b) of both corollaries, corresponding to Theorem 8.1.(a).

The above change has the following implications. First of all, it still holds, as shown in Theorem 6.2, that every i.d.view is $g$-morphic to a direct view. This implies that questions like existence of an i.d.view can be addressed by considering only direct views. However, for questions regarding behavior, direct views may not be sufficient. As shown in the example above in Fig.1, and also Fig.13 of the paper, there are some i.d.views that are behaviorally different from any direct view. 